\title{
Joint desirability foundations of social choice and opinion pooling
}

\author{
Arianna Casanova $^{1}$ (D) $\cdot$ Enrique Miranda ${ }^{2} \cdot$ Marco Zaffalon $^{1}$
}

Accepted: 18 February 2021 / Published online: 19 April 2021

(C) The Author(s) 2021

\begin{abstract}
We develop joint foundations for the fields of social choice and opinion pooling using coherent sets of desirable gambles, a general uncertainty model that allows to encompass both complete and incomplete preferences. This leads on the one hand to a new perspective of traditional results of social choice (in particular Arrow's theorem as well as sufficient conditions for the existence of an oligarchy and democracy) and on the other hand to using the same framework to analyse opinion pooling. In particular, we argue that weak Pareto (unanimity) should be given the status of a rationality requirement and use this to discuss the aggregation of experts' opinions based on probability and (state-independent) utility, showing some inherent limitation of this framework, with implications for statistics. The connection between our results and earlier work in the literature is also discussed.
\end{abstract}

Keywords Arrow · Desirability · Social choice $\cdot$ Opinion pooling · Imprecise probabilities · Coherence

\section{Introduction}

This work is concerned with the question of aggregating beliefs (probabilities) and values (utilities) of a given number of rational subjects. The problem is of a foundational and philosophical nature; at the same time it has concrete statistical implications given that in applications we often want to aggregate information coming from different sources, or even predictions of different models.

Arianna Casanova

arianna@idsia.ch

Enrique Miranda

mirandaenrique@uniovi.es

Marco Zaffalon

zaffalon@idsia.ch

1 Istituto Dalle Molle di Studi sull'Intelligenza Artificiale (IDSIA), Lugano, Switzerland

2 University of Oviedo, Oviedo, Spain 
Not surprisingly, the problem has a long history in the literature. A prominent example can be found in the voting context of social choice theory [19, 47], which aims at defining social functions that best represent the preferences of a group of rational voters. In this framework, the celebrated Arrow's theorem ([2]) establishes limits to what is possible to do in order to satisfy some reasonable properties for a voting system, while avoiding dictatorial solutions; these limits are particularly severe with complete preferences. Social choice theory is concerned with preferences over simple options (such as candidates to an election). As such it is not directly concerned with questions of probability. The related research field of probabilistic opinion pooling is instead concerned with finding a model that best 'summarises' a given number of probabilistic beliefs [30]. Stewart and Ojea Quintana analyse at some length the difficulties of precise probabilistic approaches to opinion pooling, and concede that imprecise probability may have a say on the matter for certain interpretations of pooling [48]. A strong appeal for imprecise probability in opinion pooling was advocated long ago by Walley along with a deep analysis of the subject in [53].

Although social choice and opinion pooling have a number of distinct features, they can also be gathered together under the umbrella of beliefs aggregation: indeed both regard the aggregation of the belief models of a number of voters or experts, being in the shape of preferences over a number of alternatives (in social choice) or uncertainty models about some experiment (in opinion pooling). Thus, it may be useful to give them a common treatment that can later be particularised to each problem so as to deal with its specificities. In order to accomplish this, we would need a belief model that can include as particular cases (possibly incomplete) preference relations as well as (sets of) probability measures. It turns out that such a role can be played by the theory of sets of desirable gambles [57] (see also [5, Chapter 1] for a recent overview). It has its roots in the behavioural theory of imprecise probabilities, which was later much developed by Walley [54], and it has the advantage of being sufficiently general while at the same time easier to work with than sets of probability measures or coherent lower previsions. In particular, it can deal more effectively than other models with the problem of sets of measure zero [34].

One additional advantage of working with sets of desirable gambles is that they allow us to make judgements of incompleteness and incomparability; this is relevant since the completeness axiom has been widely criticised in the literature [21, 23, 31]. As we shall see in this paper, this will be key to escape some of the constraints that are encountered in the precise case, such as Arrow's impossibility theorem in social choice or the impossibility of preserving independence in the case of linear opinion pooling. In addition, sets of desirable gambles permit us to simultaneously deal with considerations of beliefs and values [60] and to consider any domain and possibility space [61]. This shall allow us to endorse imprecise probabilities as a model that is not only more natural but also more flexible so as to have useful mathematical properties.

On this basis, after giving some preliminary notions of sets of desirable gambles in Section 2, we formulate social choice in desirability terms in Section 3. Then we work out the foundations of such a reformulated theory in Section 4, showing how some traditional results in social choice [25, 56] transform in our setting: dictatorship (Section 4.1), oligarchy (Section 4.2), and democracy (Section 4.3). Among other things, we show that the only possible way to obtain a democracy in this context is by avoiding conflicts among individuals, endorsing only those opinions that are shared by all subjects: stated differently, incompleteness turns out to be necessary for democracy.

In Section 5, we analyse more deeply the relation between social rules and the notion of coherence in desirability. We show that the property of weak Pareto (unanimity) is 
equivalent to the possibility to have social rules that are logically consistent with the voters' opinions, and that we call coherent social rules.

Imposing this notion, in Section 6 we show that coherent and complete social rules are mixtures of the voters' opinions. We use this result to analyse a special case of linear pooling where probabilities and utilities are independently specified, as it is usually the case in practical applications. We show that dictatorship can again be avoided by considering an imprecise aggregated set.

In order to illustrate further the different results, we have detailed an example in Section 7, where we also provide a graphical summary of the main results in the paper.

Section 8 discusses in some detail earlier works that appear to be more tightly related than others to the work we have carried out in this paper: in particular, the work by Pini, Rossi, Venable and Walsh on social choice in [43]; Walley's work on the aggregation of beliefs in [53]; and Stewart and Ojea Quintana's [48] as well as Dietrich and List's [14, 15] work on opinion pooling.

We conclude with some additional comments and perspective for future research in Section 9.

Finally, Appendix A gives an equivalent representation of social rules, that puts the focus on profiles of voters for which a gamble is desirable for the group, Appendix B reformulates the main results of the paper, for completeness, in terms of sets of probability measures or coherent lower previsions (lower expectation functionals). Appendix $\mathrm{C}$ gathers all the proofs of the results in the paper.

\section{Coherent sets of desirable gambles}

We start by introducing the necessary notation and basic definitions from the behavioural theory of imprecise probabilities. For additional comments, we refer to [54] and [5].

The main modelling tool from the theory that we shall use in this paper is that of a gamble:

Definition 1 (Gamble) Given a (possibly infinite) possibility space $\Omega$, a gamble $f: \Omega \rightarrow$ $\mathbb{R}$ is a bounded real-valued function on $\Omega$.

A gamble is interpreted as an uncertain reward in a linear utility scale. ${ }^{1}$ A subject might desire a gamble or not, depending on the information she has about the experiment whose possible outcomes are the elements of $\Omega$. We denote the set of all gambles on $\Omega$ by $\mathcal{L}(\Omega)$, or more simply by $\mathcal{L}$ when there is no possible ambiguity. We also let $\mathcal{L}^{+}(\Omega):=\{f \in$ $\mathcal{L}(\Omega): f \geq 0, f \neq 0\}$, or simply $\mathcal{L}^{+}$, denote the subset of positive gambles, and similarly $\mathcal{L}^{-}(\Omega):=\{f \in \mathcal{L}(\Omega): f \leq 0, f \neq 0\}$, or simply $\mathcal{L}^{-}$, denote the subset of negative gambles. The gambles in $\mathcal{L}^{+}$are gambles that should always be desired, since they may increase the wealth with no risk of decreasing it, while those in $\mathcal{L}^{-}$should never be desired. As a consequence of the linearity of our utility scale we assume also that if a subject is disposed to take the transaction represented by the gambles $f$ and $g$, then she should also be disposed to accept both transactions simultaneously, i.e., the gamble $f+g$ should also be desirable to her.

\footnotetext{
${ }^{1}$ Linearity means that if we are willing to take gambles $f_{1}$ and $f_{2}$, then we are also willing to take gamble $\lambda_{1} f_{1}+\lambda_{2} f_{2}$ for any real constants $\lambda_{1}, \lambda_{2}>0$ (check D3, D4 in Definition 2). See [54, Section 2.2.2] and [61, Section 4] for background and a justification of the linearity assumption.
} 
More generally, we can consider the notion of a coherent set of desirable gambles.

Definition 2 (Coherent set of desirable gambles) We say that a subset $\mathcal{D}$ of $\mathcal{L}(\Omega)$ is a coherent set of desirable gambles if and only if $\mathcal{D}$ satisfies the following properties:

D1. $\mathcal{L}^{+} \subseteq \mathcal{D}$ [Accepting Partial Gains];

D2. $0 \notin \mathcal{D}$ [Avoiding Null Gain];

D3. $f, g \in \mathcal{D} \Rightarrow f+g \in \mathcal{D}$ [Additivity];

D4. $f \in \mathcal{D}, \lambda>0 \Rightarrow \lambda f \in \mathcal{D}$ [Positive Homogeneity].

This leads to the concept of natural extension defined starting from the conic hull operator:

Definition 3 (Conic and convex hull) Given a set $\mathcal{K} \subseteq \mathcal{L}(\Omega)$, we let

$$
\operatorname{posi}(\mathcal{K}):=\left\{\sum_{j=1}^{r} \lambda_{j} f_{j}: f_{j} \in \mathcal{K}, \lambda_{j}>0, r \geq 1\right\}
$$

denote the conic hull of the original set and

$$
\operatorname{ch}(\mathcal{K}):=\left\{\sum_{j=1}^{r} \lambda_{j} f_{j}: f_{j} \in \mathcal{K}, \lambda_{j} \geq 0, \sum_{j=1}^{r} \lambda_{j}=1, r \geq 1\right\},
$$

denote the convex hull of the original set.

Definition 4 (Natural extension for gambles) Given a set $\mathcal{K} \subseteq \mathcal{L}(\Omega)$, we call $\mathcal{D}:=$ $\operatorname{posi}\left(\mathcal{K} \cup \mathcal{L}^{+}\right)$its natural extension.

Hence the natural extension is the set of all gambles that should be regarded as desirable once it is stated that those in $\mathcal{K}$ are. Note that the natural extension is coherent if and only if it avoids null gain. More generally speaking:

Definition 5 (Avoiding partial loss for gambles) We say that a set $\mathcal{K} \subseteq \mathcal{L}(\Omega)$ avoids partial loss if and only if its natural extension avoids null gain:

$$
0 \notin \operatorname{posi}\left(\mathcal{K} \cup \mathcal{L}^{+}\right) .
$$

Equivalently, the avoiding partial loss condition means that a certain set of gambles has a coherent superset. The intersection of all its coherent supersets is just its natural extension.

Definition 6 (Measurable gambles) Given a partition $\mathcal{B}$ of $\Omega$, we say that a gamble $f$ on $\Omega$ is $\mathcal{B}$-measurable if and only if it is actually a function on $\mathcal{B}$ :

$$
(\forall B \in \mathcal{B})\left(\forall \omega, \omega^{\prime} \in B\right) f(\omega)=f\left(\omega^{\prime}\right) .
$$

We shall denote by $\mathcal{L}_{\mathcal{B}}(\Omega)$ the subset of $\mathcal{L}(\Omega)$ given by the $\mathcal{B}$-measurable gambles. Note that there is a one-to-one correspondence between $\mathcal{L}_{\mathcal{B}}(\Omega)$ and $\mathcal{L}(\mathcal{B})$.

Definition 7 (Conditional gambles) Given a non-empty set $B \subseteq \Omega$, we say that a gamble $f$ on $\Omega$ is conditional on $B$ if and only if it is zero outside $B: f=B f$, where $B$ is also 
used to denote the indicator gamble of the event $B$, given by

$$
B(\omega)= \begin{cases}1 & \text { if } \omega \in B \\ 0 & \text { otherwise }\end{cases}
$$

We shall denote by $\mathcal{L}(\Omega) \mid B$ the subset of $\mathcal{L}(\Omega)$ made of gambles that are conditional on $B \subseteq \Omega$. Note that there is a one-to-one correspondence between $\mathcal{L}(\Omega) \mid B$ and $\mathcal{L}(B)$.

Definition 8 (Coherence relative to a set of gambles) We shall say that a set $\mathcal{D} \subseteq \mathcal{L}_{\mathcal{B}}(\Omega)$ is coherent relative to $\mathcal{L}_{\mathcal{B}}(\Omega)$ when the set $\mathcal{D}^{\prime} \subseteq \mathcal{L}(\mathcal{B})$ that we can make a one-to-one correspondence with, is coherent. In a similar way, we shall say that $\mathcal{D} \subseteq \mathcal{L}(\Omega) \mid B$ is coherent relative to $\mathcal{L}(\Omega) \mid B$ when the set $\mathcal{D}^{\prime} \subseteq \mathcal{L}(B)$ that we can make a one-to-one correspondence with, is coherent.

Here the intuition is that it often makes sense to restrict the attention to a subset of gambles with a certain property, like, e.g., the gambles conditional on an event $B: \mathcal{L}(\Omega) \mid B$ (i.e., equal to zero outside $B$ ). These gambles can equivalently be represented as gambles in $\mathcal{L}(B)$ (in the same way as a conditional probability can be regarded as an unconditional probability in a restricted space). In this representation a subset $\mathcal{D}^{\prime}$ of $\mathcal{L}(B)$ can well be coherent. Its counterpart $\mathcal{D}$ in $\mathcal{L}(\Omega) \mid B$ obviously maintains this property of rationality too, and we stress this by saying that it is coherent relative to $\mathcal{L}(\Omega) \mid B$; in general it is not coherent relative to the whole set of gambles $\mathcal{L}(\Omega)$. It is so in trivial cases: coherence of $\mathcal{D}$ relative to $\mathcal{L}(\Omega) \mid \Omega$ (as well as coherence of $\mathcal{D}$ relative to $\mathcal{L}_{\Omega}(\Omega)$ ) is just coherence of $\mathcal{D}$.

Definition 9 (Marginal set of gambles) Let $\mathcal{D} \subseteq \mathcal{L}(\Omega)$ be a coherent set of desirable gambles and consider a partition $\mathcal{B}$ of $\Omega$. The $\mathcal{B}$-marginal of $\mathcal{D}$ is the $\operatorname{set} \mathcal{D}_{\mathcal{B}}:=\mathcal{D} \cap \mathcal{L}_{\mathcal{B}}(\Omega)$.

It follows that $\mathcal{D}_{\mathcal{B}}$ is coherent relative to $\mathcal{L}_{\mathcal{B}}$.

Definition 10 (Conditional set of gambles) Let $\mathcal{D} \subseteq \mathcal{L}(\Omega)$ be a coherent set of desirable gambles and consider a non-empty set $B \subseteq \Omega$. The $B$-conditional of $\mathcal{D}$ is the $\operatorname{set} \mathcal{D} \mid B:=$ $\mathcal{D} \cap \mathcal{L}(\Omega) \mid B$.

It follows that $\mathcal{D} \mid B$ is coherent relative to $\mathcal{L}(\Omega) \mid B$.

A coherent set of desirable gambles encompasses a probabilistic model for $\Omega$ made of lower and upper expectations:

Definition 11 (Coherent lower and upper prevision) Let $\mathcal{D}$ be a coherent set of desirable gambles in $\mathcal{L}$. For all $f \in \mathcal{L}$, let:

$$
\underline{P}_{\mathcal{D}}(f):=\sup \{\mu \in \mathbb{R}: f-\mu \in \mathcal{D}\},
$$

where, with a certain abuse of notation, for any real number $\mu$ we also use the symbol $\mu$ to denote the constant gamble given by $g(\omega)=\mu$ for every $\omega \in \Omega$.

The value $\underline{P}_{\mathcal{D}}(f)$ is called the lower prevision of $f$. The conjugate value given by $\bar{P}_{\mathcal{D}}(f):=-\underline{P}_{\mathcal{D}}(-f)$ is called the upper prevision of $f$. The functionals $\underline{P}_{\mathcal{D}}, \bar{P}_{\mathcal{D}}: \mathcal{L} \rightarrow$ $\mathbb{R}$ are respectively called a coherent lower prevision and a coherent upper prevision. If $\underline{P}_{\mathcal{D}}=\bar{P}_{\mathcal{D}}$ for some $f \in \mathcal{L}$, then we call the common value the prevision of $f$ and we denote it by $P_{\mathcal{D}}(f)$. If this happens for all $f \in \mathcal{L}$ then we call the functional $P_{\mathcal{D}}$ a linear prevision. 
Alternatively, a coherent lower prevision can be defined directly on $\mathcal{L}$ without assessing first a coherent set of desirable gambles $\mathcal{D}$ and applying then (1); in that case, we shall use the notation $\underline{P}$. A coherent lower prevision $\underline{P}$ has a set of dominating linear previsions:

$$
\mathcal{M}(\underline{P}):=\{P \text { linear prevision : }(\forall f \in \mathcal{L}) P(f) \geq \underline{P}(f)\}
$$

which turns out to be closed and convex. Each linear prevision is in a one-to-one correspondence with a finitely additive probability, which can be obtained by making the restriction of the linear prevision to indicators of events. As a consequence, we can regard $\mathcal{M}(\underline{P})$ also as a set of probabilities (a so-called credal set).

There is a one-to-one correspondence between coherent lower previsions and a special type of coherent desirable sets:

Definition 12 (Strict desirability) A coherent set of gambles $\mathcal{D}$ is said to be strictly desirable if and only if it satisfies $\left(\forall f \in \mathcal{D} \backslash \mathcal{L}^{+}\right)(\exists \delta>0) f-\delta \in \mathcal{D}$.

We shall employ the notation $\mathcal{D}_{\triangleright}$ for coherent sets of strictly desirables, to differentiate them from the general case of coherent sets of desirable gambles. Given a coherent lower prevision $\underline{P}$, the set:

$$
\mathcal{D}_{\triangleright}:=\mathcal{L}^{+} \cup\{f \in \mathcal{L}: \underline{P}(f)>0\},
$$

is coherent and strictly desirable and moreover induces $\underline{P}$ through (1).

This one-to-one correspondence does not hold for arbitrary coherent sets of desirable gambles, in the sense that several different coherent sets of desirable gambles $\mathcal{D}$ may induce the same coherent lower prevision $\underline{P}$ by means of (1). All of them are associated with the same set of strictly desirable gambles $\mathcal{D}_{\triangleright}$, which can be recovered directly from $\mathcal{D}$ by

$$
\mathcal{D}_{\triangleright}:=\mathcal{L}^{+} \cup\{f:(\exists \varepsilon>0) f-\varepsilon \in \mathcal{D}\} .
$$

Finally, we consider the most informative cases of coherent sets of gambles:

Definition 13 (Maximal coherent set of gambles) A coherent set of desirable gambles $\mathcal{D}$ is called maximal if and only if

$$
(\forall f \in \mathcal{L} \backslash\{0\}) f \notin \mathcal{D} \Rightarrow-f \in \mathcal{D} \text {. }
$$

A maximal set of desirable gambles has no coherent superset, and, conversely, any coherent set of desirable gambles is the intersection of its coherent maximal supersets. In other words, coherent sets of desirable gambles constitute a strong belief structure, where the maximal elements are indeed the maximal coherent sets of desirable gambles; see $[11,54]$ and [5, Chapter 1] for further information.

A maximal coherent set of desirable gambles induces a linear prevision by means of (1). Nevertheless, a linear prevision $P$ may be induced by several different coherent sets of desirable gambles. It may be interesting then to consider the least informative one:

Definition 14 (Maximal strict desirability) A strictly desirable set of gambles $\mathcal{D}_{\triangleright}$ is called maximally strictly desirable if and only if there is a linear prevision $P$ that determines $\mathcal{D}_{\triangleright}$ by means of (3). 


\section{Desirability formulation of social choice}

Having introduced sets of gambles allows us to reformulate the main concepts in social choice theory with them (we refer to Appendix B for the counterpart of the next results in terms of coherent lower previsions).

We start with a set of $n$ 'voters' $\mathcal{H}$, each of them with its own uncertainty model concerning a possibility space $\mathcal{S}$. In our setting it is straightforward to deal also with utility considerations, by assuming that voters have in addition values on a set of prizes $\mathcal{X}$ and by taking the new 'possibility' space to be $\mathcal{Z}:=\mathcal{S} \times \mathcal{X}[60,61]$. In fact this work lives at such a level of generality; however the reader need not be concerned with it, given that technically $\mathcal{Z}$ is treated just as a possibility space (we shall need to be explicit about this only in Section 6).

Let us next define voters' profiles.

Definition 15 (Profiles) Let $\mathbb{D}$ be the set of coherent sets of desirable gambles on $\mathcal{Z} . \mathbb{D}^{n}$, the $n$-times Cartesian product of $\mathbb{D}$, is the set of logically possible profiles of individual sets of desirable gambles.

A profile of coherent sets of desirable gambles is thus a vector $\left[\mathcal{D}_{i}\right]_{i \in \mathcal{H}} \in \mathbb{D}^{n}$. To keep the notation simple, we shall often denote it simply by $\left[\mathcal{D}_{i}\right]$.

With this formalism we can re-define also the concept of a social welfare function, which we rename in this context as social rule, and all the properties that it must satisfy for showing the results (for the original concepts and definitions see [56]).

Definition 16 (Social rule) A social rule $\Gamma$ is a function from a set $\mathcal{A} \subseteq \mathbb{D}^{n}$ to a coherent set of desirable gambles (social coherent set of desirable gambles). Its domain $\mathcal{A}$ is called the admissible set of profiles for $\Gamma$.

Social coherent sets of desirable gambles remain unsubscripted while subscripts distinguish individuals' coherent sets of desirable gambles. Note that our social rules are in particular applicable when the beliefs are modelled by probability measures, coherent lower previsions, or other uncertainty models, such as belief functions; it suffices to make the correspondence with coherent sets of desirable gambles in (1). In this respect, we should also remark here that a coherent set of desirable gambles can be seen as equivalent to a consistent set of binary preferences, in the sense that a gamble is desirable if and only if it is considered to be preferable to the status quo, and a gamble $f$ is preferred to $g$ if and only if their difference is considered desirable. Therefore, the coherent set of desirable gambles of a voter can be obtained simply as the natural extension of the gambles the voter deems desirable by making binary comparisons between the alternatives; but it may also be more precise and include additional information.

It is exactly this degree of generality that allows us to handle issues of opinion pooling, because it allows us to express beliefs on $\mathcal{Z}$ that are more nuanced than simple preferences on alternatives. To see a practical example of social rules constructed for voters that express their beliefs with different formalisms, both precise and imprecise, see Section 7.

Indeed, with this formalism, we can naturally treat incomplete preferences working with coherent sets of desirable gambles that are not necessarily maximal. Recall that, because of the properties of coherence, for any profile $\left[\mathcal{D}_{i}\right]$ the set $\Gamma\left(\left[\mathcal{D}_{i}\right]\right)$ is the intersection of a family of maximal sets of desirable gambles. 
Example 1 As a simple running example in order to clarify some of the notions that follow, consider a set of $n=2$ voters, and a possibility space $\mathcal{S}$ and a set of prizes $\mathcal{X}$ also with cardinality two. Then following are four instances of social rules (for any possible choice of coherent sets of desirable gambles $\left.\mathcal{D}_{1}, \mathcal{D}_{2}\right)$ :

$\circ \quad \Gamma_{1}\left(\mathcal{D}_{1}, \mathcal{D}_{2}\right)=\mathcal{D}_{1}$

○ $\Gamma_{2}\left(\mathcal{D}_{1}, \mathcal{D}_{2}\right)=\mathcal{D}_{1} \cap \mathcal{D}_{2}$.

- $\quad \Gamma_{3}\left(\mathcal{D}_{1}, \mathcal{D}_{2}\right)=\mathcal{M}_{2}$, where $\mathcal{M}_{2}$ is a maximally coherent set of gambles that includes $\mathcal{D}_{2}$.

$\circ \quad \Gamma_{4}\left(\mathcal{D}_{1}, \mathcal{D}_{2}\right)=\operatorname{posi}\left(\left\{\mathcal{D}_{2} \backslash \mathcal{D}_{1}\right\} \cup \mathcal{L}^{+}\right) . \diamond$

Equivalently, a social rule $\Gamma$ can be represented as a bundle of functions $\left\{\Gamma_{f}: f \in \mathcal{L}\right\}$, where $\Gamma_{f}$ identifies those profiles for which the gamble $f$ belongs to the aggregated set. For a detailed account of this representation and the translation of the concepts that follow into this formalism, we refer to Appendix A.

Next we consider a number of additional properties that a social rule may satisfy. From our formulation, such a rule turns the, possibly imprecise, assessments of a number of voters (such as those that lead to a coherent lower prevision by means of (1)) into an aggregated assessment, which may be imprecise too. As particular cases of interest, we may consider the case when the aggregated set represents precise assessments:

Definition 17 (Completeness) A social rule $\Gamma$ satisfies completeness if and only if $\Gamma\left(\left[\mathcal{D}_{i}\right]\right)$ is a maximal set of gambles for every profile $\left[\mathcal{D}_{i}\right]$, and it satisfies strict completeness if and only if $\Gamma\left(\left[\mathcal{D}_{i}\right]\right)$ is a maximally strictly desirable set of gambles for every profile $\left[\mathcal{D}_{i}\right]$.

Another important assumption we shall consider is that $\Gamma$ can be applied to any profile of voters:

Definition 18 (Unlimited domain) A social rule $\Gamma$ satisfies unlimited domain if and only if $\mathcal{A}=\mathbb{D}^{n}$ where $\mathcal{A}$ denotes the admissible set of profiles. It is said to satisfy unlimited maximal domain when $\mathcal{A}=\hat{\mathbb{D}}^{n}$, the set of profiles constituted by maximal coherent sets of desirable gambles.

In the social rules in Example 1 we shall assume that they satisfy unlimited domain. Then we can see that only $\Gamma_{3}$ is complete, because $\Gamma_{1}, \Gamma_{2}, \Gamma_{4}$ will not return a maximal set of gambles if for instance $\mathcal{D}_{1}=\mathcal{D}_{2}=\mathcal{L}^{+}(\mathcal{Z})$.

Next we consider a property called independence of irrelevant alternatives, which shall be instrumental in characterising a number of social rules.

Definition 19 (Independence of irrelevant alternatives) A social rule is independent of irrelevant alternatives if and only if $(\forall f \in \mathcal{L})\left(\forall\left[\mathcal{D}_{i}\right],\left[\mathcal{D}_{i}^{\prime}\right] \in \mathcal{A}\right)$

$$
\left((\forall i \in \mathcal{H})\left(f \in \mathcal{D}_{i} \Leftrightarrow f \in \mathcal{D}_{i}^{\prime}\right)\right) f \in \Gamma\left(\left[\mathcal{D}_{i}\right]\right) \Leftrightarrow f \in \Gamma\left(\left[\mathcal{D}_{i}^{\prime}\right]\right) .
$$

The interpretation of this property is that whether a gamble $f$ belongs to the aggregated decision set depends only on which voters are endorsing $f$ (but note that this may depend on the gamble $f$ we consider, in the sense that two gambles $f, g$ may belong to the same elements in the profile and one may belong to the aggregated set while the other one is not).

If we consider the social rules in the running example, we observe that $\Gamma_{1}$ and $\Gamma_{2}$ satisfy independence of irrelevant alternatives, because their definition depends only on which sets 
in the profile include the gamble; but $\Gamma_{3}$ and $\Gamma_{4}$ do not if for example both satisfy also unlimited domain. To see this for $\Gamma_{3}$, consider a profile $\left[\mathcal{D}_{1}, \mathcal{D}_{2}\right]$ where $\mathcal{D}_{2}$ is not maximal, and take $f$ such that $f,-f \notin \mathcal{D}_{2}$. If for instance $f \in \Gamma\left(\left[\mathcal{D}_{1}, \mathcal{D}_{2}\right]\right)$, then we can consider another profile $\left[\mathcal{D}_{i}^{\prime}\right]$ where $\mathcal{D}_{1}^{\prime}=\mathcal{D}_{1}$ and $\mathcal{D}_{2}^{\prime}$ is a maximal set of desirable gambles that includes $-f$. It follows that $-f \in \Gamma\left(\left[\mathcal{D}_{i}^{\prime}\right]\right)$ and as a consequence $f \notin \Gamma\left(\left[\mathcal{D}_{i}^{\prime}\right]\right)$, thus violating independence of irrelevant alternatives.

For $\Gamma_{4}$ instead, consider a gamble $f \notin \mathcal{L}^{+} \cup \mathcal{L}^{-} \cup\{0\}$, fix $\varepsilon>0$ such that $f-\varepsilon \notin$ $\left(\mathcal{L}^{-} \cup\{0\}\right)$ and let $\mathcal{D}_{1}=\operatorname{posi}\left(\{f\} \cup \mathcal{L}^{+}\right), \mathcal{D}_{2}=\operatorname{posi}\left(\{f-\varepsilon\} \cup \mathcal{L}^{+}\right)$. Then $f \in \operatorname{posi}\left(\left(\mathcal{D}_{2} \backslash\right.\right.$ $\left.\left.\mathcal{D}_{1}\right) \cup \mathcal{L}^{+}\right)=\Gamma\left(\left[\mathcal{D}_{1}, \mathcal{D}_{2}\right]\right)$. If instead we take the profile $\mathcal{D}_{1}^{\prime}=\mathcal{D}_{2}^{\prime}=\mathcal{D}_{2}$, then we get that $\Gamma\left(\left[\mathcal{D}_{1}^{\prime}, \mathcal{D}_{2}^{\prime}\right]\right)=\mathcal{L}^{+}$, whence $f \notin \Gamma\left(\left[\mathcal{D}_{i}^{\prime}\right]\right)$ even if in both cases $f$ belongs to both sets in the profile. Therefore, $\Gamma_{4}$ does not satisfy independence of irrelevant alternatives.

The next condition states that if a gamble is deemed desirable by all the voters, then it should belong to the aggregated set:

Definition 20 (Weak Pareto) A social rule $\Gamma$ satisfies weak Pareto if and only if

$$
\left(\forall\left[\mathcal{D}_{i}\right] \in \mathcal{A}\right) \cap_{i \in \mathcal{H}} \mathcal{D}_{i} \subseteq \Gamma\left(\left[\mathcal{D}_{i}\right]\right)
$$

This axiom is sometimes referred to as unanimity [43]. Note that since the intersection of a family of coherent sets of desirable gambles is again a coherent set of desirable gambles, this definition is consistent. We can also see that all the social rules in our running example, except $\Gamma_{4}$, satisfy weak Pareto. To see a profile on which $\Gamma_{4}$ does not satisfy weak Pareto, consider the profile $\left[\mathcal{D}_{1}^{\prime}, \mathcal{D}_{2}^{\prime}\right]$ used to show that $\Gamma_{4}$ does not satisfy independence of irrelevant alternatives. It also follows from the notion of coherence for sets of desirable gambles that if $\Gamma$ is a social rule that satisfies weak Pareto, then for any profile $\left[\mathcal{D}_{i}\right], \Gamma\left(\left[\mathcal{D}_{i}\right]\right)$ is an intersection of a family of maximal sets that include $\cap_{i} \mathcal{D}_{i}$.

In this paper, we shall analyse situations where the overall decision is determined or influenced by the behaviour of a number of particular voters. We shall consider a number of cases.

Definition 21 (Almost decisive set of individuals) Given a social rule $\Gamma$, a set of individuals $\mathcal{G} \subseteq \mathcal{H}$ is almost decisive for a gamble $f$ when

$$
\left(\forall\left[\mathcal{D}_{i}\right] \in \mathcal{A}\right) f \in \cap_{i \in \mathcal{G}} \mathcal{D}_{i} \text { and } f \notin \cup_{i \notin \mathcal{G}} \mathcal{D}_{i} \Rightarrow f \in \Gamma\left(\left[\mathcal{D}_{i}\right]\right)
$$

It is called almost decisive when it is almost decisive for every gamble $f$.

With respect to the social rules in the running example, for $\Gamma_{1}$ the first voter is almost decisive, while the second is not; for $\Gamma_{2}$, the set $\{1,2\}$ is almost decisive, but $\{1\}$ or $\{2\}$ separately are not; and for $\Gamma_{3}$ and $\Gamma_{4}$, the second voter is almost decisive, while the first one is not.

Note that when $\mathcal{G}=\mathcal{H}$ almost decisiveness reduces to $\Gamma$ satisfying weak Pareto, while if $\mathcal{G}$ is a proper subset of $\mathcal{H}$, we may assume without loss of generality that $f \notin \mathcal{L}^{+} \cup \mathcal{L}^{-} \cup\{0\}$, considering that any $f \in \mathcal{L}^{+}$must belong to $\cap_{i \notin \mathcal{G}} \mathcal{D}_{i}$ and that any $f \in \mathcal{L}^{-} \cup\{0\}$ cannot belong to $\mathcal{D}_{i}$ for any $i \in \mathcal{G}$.

On the other hand, if the social rule satisfies independence of irrelevant alternatives, we can easily deduce judgements of almost decisiveness: if we consider a profile $\left[\mathcal{D}_{i}\right]$ such that $f \in \Gamma\left(\left[\mathcal{D}_{i}\right]\right)$, then it follows that $\mathcal{G}=\left\{i: f \in \mathcal{D}_{i}\right\}$ is almost decisive with respect to $f$.

A slightly stronger notion is the following: 
Definition 22 (Decisive set of individuals) Given a social rule $\Gamma$, a set of individuals $\mathcal{G} \subseteq$ $\mathcal{H}$ is decisive for a gamble $f$ if and only if

$$
\left(\forall\left[\mathcal{D}_{i}\right] \in \mathcal{A}\right) f \in \cap_{i \in \mathcal{G}} \mathcal{D}_{i} \Rightarrow f \in \Gamma\left(\left[\mathcal{D}_{i}\right]\right),
$$

and it is called decisive when it is decisive for every gamble $f$.

In our running example, the first voter is decisive for $\Gamma_{1}$ and the second is decisive for $\Gamma_{3}$. Hence, since in this example we have only two voters, they correspond to what we shall call next a dictator. Notice that instead for $\Gamma_{4}$ the second voter is almost decisive without being decisive in case the domain $\mathcal{A}$ is large enough (for instance using the profile $\left[\mathcal{D}_{1}^{\prime}, \mathcal{D}_{2}^{\prime}\right]$ used to show that $\Gamma_{4}$ does not satisfy independence of irrelevant alternatives).

Intuitively, if a group $\mathcal{G}$ is almost decisive for a gamble $f$ it should also be decisive for $f$; this would hold for instance if we require that the social rule is monotone for every $f$. Indeed, for a monotone social rule $\Gamma$, if the set of voters that considers $f$ desirable in a profile $\left[\mathcal{D}_{i}^{\prime}\right]$ includes all those that consider it desirable in a profile $\left[\mathcal{D}_{i}\right]$, then, if $f \in$ $\Gamma\left(\left[\mathcal{D}_{i}\right]\right) \Rightarrow f \in \Gamma\left(\left[\mathcal{D}_{i}^{\prime}\right]\right) .^{2}$ As we shall see in Lemma 1, we can guarantee such a property when weak Pareto and independence of irrelevant alternatives are satisfied.

As one extreme version of decisiveness, we have the case where the decisive group consists of only one voter.

Definition 23 (Dictatorship) An individual $i \in \mathcal{H}$ is a dictator for a social rule $\Gamma$ if and only if $\{i\}$ is decisive.

This means that $\mathcal{D}_{i} \subseteq \Gamma\left(\left[\mathcal{D}_{j}\right]\right)$ for any profile $\left[\mathcal{D}_{j}\right]$. Note that the two sets need not coincide: dictatorship means that those gambles that are considered desirable by voter $i$ must also be considered desirable in the overall assessment, but the latter may include others, meaning that $\Gamma\left(\left[\mathcal{D}_{j}\right]\right)$ may be a strict superset. This is a difference with the notion of strong dictator we shall discuss in Section 8.1. One particular case where the two sets will necessarily coincide is when $\mathcal{D}_{i}$ is a maximal set of gambles, because these cannot be strictly included in any other coherent set of gambles.

The above reasoning also means that, if the assumption of unlimited domain is satisfied, there can be at most one dictator: for given two different dictators $j_{1} \neq j_{2}$, it should be $\mathcal{D}_{j_{1}} \cup \mathcal{D}_{j_{2}} \subseteq \Gamma\left(\left[\mathcal{D}_{i}\right]\right)$, and given two different maximal sets of gambles $\mathcal{D}_{j_{1}}, \mathcal{D}_{j_{2}}$ their union has no coherent superset.

\section{Desirability foundations of social choice}

Let us detail now how the traditional results in social choice transform in the case of desirability.

\subsection{Dictatorship}

We shall now establish a version of Arrow's theorem for the case where the voters' preferences are assessed by means of coherent sets of desirable gambles (for a version of Arrow's theorem using preference profiles see $[19,47])$. The use of 'field expansion' and 'group

\footnotetext{
${ }^{2}$ This condition is sometimes referred to in the literature with the term non-negative responsiveness [3, 50]; our monotonicity condition is simply a finitary application of this principle.
} 
contraction' lemma in the proof of the theorem, is one of the classical ways to prove it (see $[4,25,47])$. The first one shows, somewhat surprisingly, that under some assumptions the conditions of being almost decisive for one gamble and decisive for every gamble are equivalent; note in particular that the equivalence goes beyond the connection for one particular gamble, that follows in the case of monotone social rules.

Lemma 1 (Field expansion lemma) Assume that $|\mathcal{Z}| \geq 3$. If a social rule satisfies the following properties:

- unlimited domain or unlimited maximal domain,

- independence of irrelevant alternatives,

- weak Pareto,

and it admits a group $\mathcal{G}$ that is almost decisive for a gamble $f$, then it is decisive.

Next we show that, if in addition to the previous assumptions we have completeness, then any decisive group of voters can be contracted:

Lemma 2 (Group contraction lemma) Assume that $|\mathcal{Z}| \geq 3$. If a social rule satisfies the following properties:

- completeness,

- unlimited maximal domain,

- independence of irrelevant alternatives,

- weak Pareto,

then if a group $\mathcal{G}$ containing at least two individuals is decisive, then it contains a proper subset of individuals that are also decisive.

From these two lemmas it is just a small step to immediately derive our version for Arrow's theorem in terms of sets of desirable gambles.

Theorem 1 (Arrow's theorem) Assume that $|\mathcal{Z}| \geq 3$. Any social rule that satisfies:

- completeness,

- unlimited maximal domain,

- independence of irrelevant alternatives,

- weak Pareto,

makes one (unique) individual a dictator.

Regarding the social rules in the running example, we observe that if we restrict the set of admissible profiles of $\Gamma_{3}$ to $\hat{\mathbb{D}}^{n}$, then the resulting rule satisfies also independence of irrelevant alternatives. Hence it satisfies all the hypothesis of Theorem 1, giving rise to the dictatorship of the second voter.

Next we show that, under the assumption of unlimited maximal domain, independence of irrelevant alternatives is also necessary for the existence of a dictator:

Proposition 1 Assume that $|\mathcal{Z}| \geq 3$. Any social rule that satisfies unlimited maximal domain and that makes one (unique) individual a dictator, must satisfy independence of irrelevant alternatives. 
To see that a similar result does not hold under the assumption of unlimited domain, note that in the rule $\Gamma_{3}$ of the running example the second voter is a dictator. However, the rule does not satisfy independence of irrelevant alternatives.

Remark 1 The reason why we are not requiring unlimited domain in Lemma 2 is because, perhaps surprisingly, it is incompatible with the assumptions of weak Pareto, independence of irrelevant alternatives and completeness. To see this, consider $n=2$, and let $\mathcal{D}_{1}:=\mathcal{D}_{2}:=\mathcal{D}$ be a coherent non-maximal set. Then since $\Gamma$ satisfies completeness and weak Pareto, $\Gamma\left(\left[\mathcal{D}_{1}, \mathcal{D}_{2}\right]\right)$ should be a maximal set of gambles that strictly includes $\mathcal{D}$. Let $f \in \Gamma\left(\left[\mathcal{D}_{1}, \mathcal{D}_{2}\right]\right) \backslash \mathcal{D}$, and let us consider $\mathcal{D}^{\prime}=\operatorname{posi}(\mathcal{D} \cup\{-f\})$. Then since neither $f$ (by assumption) nor $-f$ (because $f$ belongs to the coherent superset $\Gamma\left(\left[\mathcal{D}_{1}, \mathcal{D}_{2}\right]\right)$ of $\mathcal{D}$ ) belong to $\mathcal{D}$, we deduce from Lemma 5 in Appendix $\mathrm{C}$ that $\mathcal{D}^{\prime}$ is a coherent set of desirable gambles. Now, if we take $\mathcal{D}_{1}^{\prime}=\mathcal{D}_{2}^{\prime}=\mathcal{D}^{\prime}$, it follows that $f \notin \mathcal{D}^{\prime}$, and applying independence of irrelevant alternatives we should have that $f \in \Gamma\left(\left[\mathcal{D}_{1}^{\prime}, \mathcal{D}_{2}^{\prime}\right]\right)$. But on the other hand we have that $-f \in \mathcal{D}^{\prime} \subseteq \Gamma\left(\left[\mathcal{D}_{1}^{\prime}, \mathcal{D}_{2}^{\prime}\right]\right)$ by weak Pareto, and since a coherent set of gambles cannot include both a gamble and its opposite, we arrive to a contradiction.

Since, as we shall see in Section 5, weak Pareto can be regarded as a form of coherence, this seems to indicate that, if we want the aggregated set of desirable gambles to be maximal, then we should either (a) require that all the sets in the profile are maximal, too; or (b) give up on the assumption of independence of irrelevant alternatives. The second of these paths shall be explored in Section 6 in the context of linear pooling; as we shall see in next section, the first one leads to the notion of oligarchy. $\diamond$

\subsection{Oligarchy}

As we have seen in Remark 1, the assumption of independence of irrelevant alternatives essentially prevents us from considering a social rule that aggregates imprecise profiles (that is, profiles where not all the sets $\mathcal{D}_{i}$ are maximal) into a precise one. Therefore, if we want to be able to work with imprecise (non-maximal) sets of desirable gambles and endorse the assumption of independence of irrelevant alternatives, we must renounce on the completeness (i.e. preciseness in our framework) of the aggregated set. In this section, we explore the implications of this path. As we shall prove, this leads to a general oligarchy theorem. It generalises the work of Weymark [56] considering also incompleteness in the profile of individuals (i.e. impreciseness in our framework) and [43] eliminating further restrictions on the social rule not classically imposed by the Arrowian framework. The proofs are inspired from the work of Weymark [56].

Definition 24 (Oligarchy) Given a social rule $\Gamma$, a set of individuals $\mathcal{G} \subseteq \mathcal{H}$ is an oligarchy if and only if:

O1. $\mathcal{G}$ is decisive;

O2. $(\forall f \in \mathcal{L}(\mathcal{Z}))\left(\forall\left[\mathcal{D}_{i}\right] \in \mathcal{A}\right)\left((\exists i \in \mathcal{G}) f \in \mathcal{D}_{i}\right) \Rightarrow-f \notin \Gamma\left(\left[\mathcal{D}_{i}\right]\right)$.

In other words, those gambles that are deemed desirable by all individuals of the oligarchy should also be considered desirable by the group; and the choices of the group should not contradict those of any member of the oligarchy. Equivalently, $\mathcal{G}$ is an oligarchy if and only if for any profile $\left[\mathcal{D}_{i}\right]$ it holds that

$$
\cap_{i \in \mathcal{G}} \mathcal{D}_{i} \subseteq \Gamma\left(\left[\mathcal{D}_{i}\right]\right) \subseteq \cap_{i \in \mathcal{G}}\left(-\mathcal{D}_{i}\right)^{c}
$$


The reason why we are not imposing completeness in this section is that, if $\Gamma\left(\left[\mathcal{D}_{i}\right]\right)$ was a maximal set, then for any oligarchy $\mathcal{G}$ we should have $\cup_{i \in \mathcal{G}} \mathcal{D}_{i} \subseteq \Gamma\left(\left[\mathcal{D}_{i}\right]\right)$ : indeed, if $f \in \cup_{i} \mathcal{D}_{i}$, then we cannot include $-f$ in $\Gamma\left(\left[\mathcal{D}_{i}\right]\right)$, and since this set is maximal, then it should include $f$. However, the inclusion $\cup_{i \in \mathcal{G}} \mathcal{D}_{i} \subseteq \Gamma\left(\left[\mathcal{D}_{i}\right]\right)$ is only compatible with the assumption of unlimited domain in case the oligarchy consists of only one voter, that is, in case of a dictatorship. To see this, note that if we take $|\mathcal{G}|=2$ and $\mathcal{D}_{1}, \mathcal{D}_{2}$ two different maximal sets of desirable gambles, there is no coherent superset of $\mathcal{D}_{1} \cup \mathcal{D}_{2}$.

With respect to condition $\mathrm{O} 2$, note also that, if $\mathcal{D}_{i}$ is a maximal set of gambles, then $\left(-\mathcal{D}_{i}\right)^{c}=\mathcal{D}_{i} \cup\{0\}$. It follows that if all the members of the oligarchy specify a maximal set of gambles, it follows from the definition that the decision should be $\Gamma\left(\left[\mathcal{D}_{i}\right]\right)=\cap_{i \in \mathcal{G}} \mathcal{D}_{i}$; we shall generalise this in Proposition 2 below.

The two extreme cases of oligarchy correspond to the case of $|\mathcal{G}|=1$ or $|\mathcal{G}|=n$, that is, when the oligarchy is made of just one individual or of all of them. The first case corresponds to a dictatorship. In this respect, note also that in the case of a dictator $j$ we have that $\mathcal{D}_{j} \subseteq \Gamma\left(\left[\mathcal{D}_{i}\right]\right)$, and in that case condition $\mathrm{O} 2$ follows from $\mathrm{O} 1$. The other extreme case where the oligarchy is made of all individuals will be dealt with in more detail in our next section.

It is not difficult to show that, under mild conditions, there can be at most one oligarchy:

Lemma 3 For any social rule satisfying unlimited domain or unlimited maximal domain, there can be at most one oligarchy.

Next we show that if we remove the hypothesis of completeness from Theorem 1 we can deduce that the social rule is an oligarchy.

Theorem 2 (Gibbard's theorem) Assume that $|\mathcal{Z}| \geq 3$. For any social rule $\Gamma$ that satisfies:

- unlimited domain or unlimited maximal domain,

- independence of irrelevant alternatives,

- weak Pareto,

there exists a unique oligarchy. ${ }^{3}$

Regarding the social rules in the running example, we notice that $\Gamma_{2}$ satisfies all the hypotheses of Theorem 2 . Hence it gives rise to an oligarchy that in this case corresponds to the whole society (what we shall call democracy in Section 4.3). To see an example of an oligarchy different from dictatorship or democracy, it suffices to consider the same framework of the running example, with a set of $n=3$ voters instead of two. Then the social rule $\Gamma\left(\left[\mathcal{D}_{1}, \mathcal{D}_{2}, \mathcal{D}_{3}\right]\right)=\mathcal{D}_{1} \cap \mathcal{D}_{2}$ satisfies all the hypothesis of Theorem 2 and gives rise to the oligarchy of the first two voters. A further illustration of Theorem 2 shall be given in Section 7.

We observe that in all the examples of oligarchy we have given so far we have that the social rule $\Gamma$ just picks those gambles that are deemed desirable by all members of the oligarchy: note that our version of Arrow's theorem holds under completeness and unlimited maximal domain, and as a consequence when voter $j$ acts as a dictator we have that

${ }^{3}$ Gibbard presented a theorem that is much in the spirit of ours and that can be related to incompleteness [22, p. 19] (see also [14, Section 4]). We are indebted to Franz Dietrich for this observation. 
$\Gamma\left(\left[\mathcal{D}_{i}\right]\right)=\mathcal{D}_{j}$ on every profile. This leads naturally to wonder if it is possible to obtain other forms of oligarchy, or if, on the contrary, Definition 24 is equivalent to $\Gamma\left(\left[\mathcal{D}_{i}\right]\right)=\cap_{i \in \mathcal{G}} \mathcal{D}_{i}$ (we may refer to these as strong oligarchies, as in [14]; on the other hand, when we require only condition $\mathrm{O} 1$ we have a weak oligarchy, and this is the notion considered in [21]).

Let us show first of all that, under the assumptions of Theorem 2, the oligarchy that follows is necessarily of the strong type:

Proposition 2 Let $\Gamma$ be a social rule satisfying unlimited domain (resp., unlimited maximal domain), independence of irrelevant alternatives, and weak Pareto, and let $\mathcal{G}$ be its associated oligarchy, that follows from Theorem 2. Then $\Gamma\left(\left[\mathcal{D}_{i}\right]\right)=\cap_{i \in \mathcal{G}} \mathcal{D}_{i}$.

Nevertheless, it is possible to show that, when the hypothesis of independence of irrelevant alternatives is not satisfied, one can define oligarchies that are not of the strong type. This also shows that the sufficient conditions we have established in Theorem 2 for the existence of an oligarchy are not necessary.

Example 2 Consider $n=3$, and let $\Gamma$ be the social rule given by

$$
\Gamma\left(\left[\mathcal{D}_{1}, \mathcal{D}_{2}, \mathcal{D}_{3}\right]\right)=\left(\mathcal{D}_{1} \cap \mathcal{D}_{2}\right) \cup\left(\mathcal{D}_{1 \triangleright} \cap\left(-\mathcal{D}_{2}\right)^{c}\right),
$$

where we recall that $\mathcal{D}_{1 \triangleright}=\mathcal{L}^{+} \cup\left\{f:(\exists \varepsilon>0) f-\varepsilon \in \mathcal{D}_{1}\right\}$ is the set of strictly desirable gambles associated with $\mathcal{D}_{1}$. This rule satisfies the following properties:

○ $\quad$ weak Pareto, since $\mathcal{D}_{1} \cap \mathcal{D}_{2} \cap \mathcal{D}_{3} \subseteq \mathcal{D}_{1} \cap \mathcal{D}_{2} \subseteq \Gamma\left(\left[\mathcal{D}_{i}\right]\right)$ for any profile $\left[\mathcal{D}_{i}\right]$;

○ unlimited domain (or unlimited maximal domain if needed);

- $\quad \Gamma\left(\left[\mathcal{D}_{i}\right]\right)$ is coherent: note that both $\mathcal{D}_{1} \cap \mathcal{D}_{2}$ and $\mathcal{D}_{1 \triangleright} \cap\left(-\mathcal{D}_{2}\right)^{c}$ are cones and that the sum of a gamble from $\mathcal{D}_{1}$ with a gamble from $\mathcal{D}_{1 \triangleright}$ belongs to $\mathcal{D}_{1 \triangleright}$. In other words,

$$
\begin{aligned}
\operatorname{posi}\left(\mathcal{L}^{+} \cup\left(\mathcal{D}_{1} \cap \mathcal{D}_{2}\right) \cup\left(\mathcal{D}_{1 \triangleright} \cap\left(-\mathcal{D}_{2}\right)^{c}\right)\right) & =\operatorname{posi}\left(\left(\mathcal{D}_{1} \cap \mathcal{D}_{2}\right) \cup\left(\mathcal{D}_{1 \triangleright} \cap\left(-\mathcal{D}_{2}\right)^{c}\right)\right) \\
& =\left(\mathcal{D}_{1} \cap \mathcal{D}_{2}\right) \cup\left(\mathcal{D}_{1 \triangleright} \cap\left(-\mathcal{D}_{2}\right)^{c}\right) ;
\end{aligned}
$$

○ if we consider $\mathcal{G}=\{1,2\}$, then $\Gamma$ satisfies condition $\mathrm{O} 1$ with respect to $\mathcal{G}$ by definition;

○ $\quad \Gamma\left(\left[\mathcal{D}_{i}\right]\right) \subseteq \mathcal{D}_{1}$ by construction; therefore, if $f \in \mathcal{D}_{1}$, it cannot be that $-f \in \Gamma\left(\left[\mathcal{D}_{i}\right]\right)$. Moreover, if $f \in \mathcal{D}_{2}$, then $-f \in\left(-\mathcal{D}_{2}\right)$, whence also $-f \notin \Gamma\left(\left[\mathcal{D}_{i}\right]\right)$. Therefore, $\Gamma$ satisfies condition $\mathrm{O} 2$ with respect to $\mathcal{G}$.

As a consequence, $\{1,2\}$ is an oligarchy with respect to $\Gamma$, but it is not a strong oligarchy: if we take for instance $\mathcal{D}_{2}=\mathcal{L}^{+}$, we obtain

$$
\Gamma\left(\left[\mathcal{D}_{i}\right]\right)=\mathcal{L}^{+} \cup\left\{f \in \mathcal{D}_{1 \triangleright}: f \notin\left(-\mathcal{D}_{2}\right)\right\}=\mathcal{D}_{1 \triangleright},
$$

taking into account that a coherent set of desirable gambles cannot include any gamble in $-\mathcal{D}_{2}=\mathcal{L}^{-}$. Since the set $\mathcal{D}_{1 \triangleright}$ may be a strict superset of $\mathcal{D}_{1} \cap \mathcal{D}_{2}=\mathcal{L}^{+}$, we conclude that $\Gamma$ is not a strong oligarchy. This, together with Proposition 2, implies that $\Gamma$ does not satisfy independence of irrelevant alternatives. To see this explicitly, consider a profile such that $f \in \mathcal{D}_{1 \triangleright}$ and $-f \in \mathcal{D}_{2}$. Then it follows that $f \notin \Gamma\left(\left[\mathcal{D}_{i}\right]\right)$. If now we consider $\mathcal{D}_{1}^{\prime}=\mathcal{D}_{1}, \mathcal{D}_{3}^{\prime}=\mathcal{D}_{3}$ and $\mathcal{D}_{2}^{\prime}$ a coherent set of desirable gambles that includes neither $f$ nor $-f$, it would follow from (4) that $f \in \Gamma\left(\left[\mathcal{D}_{i}^{\prime}\right]\right)$. $\diamond$

\subsection{Democracy}

By adding the property of anonymity to the axioms of Theorem 2 we obtain the polar opposite to Arrow's dictator, when the oligarchy consists of the whole society. 
Definition 25 (Anonymity) A social rule $\Gamma$ satisfies anonymity if and only if for every permutation $\sigma$ of $\mathcal{H}$ and for every profile $\left[\mathcal{D}_{i}\right]$, it holds that

$$
\Gamma\left(\left[\mathcal{D}_{i}\right]\right)=\Gamma\left(\left[\mathcal{D}_{\sigma(i)}\right]\right)
$$

This axiom corresponds to that of symmetry for Walley [53]. We see that anonymity requires the social rule to treat all individuals equally. In our running example, it is only satisfied by $\Gamma_{2}$, since we do not have in general that $\Gamma_{1}\left(\mathcal{D}_{1}, \mathcal{D}_{2}\right)=\Gamma_{1}\left(\mathcal{D}_{2}, \mathcal{D}_{1}\right)$ nor $\Gamma_{3}\left(\mathcal{D}_{1}, \mathcal{D}_{2}\right)=\Gamma_{3}\left(\mathcal{D}_{2}, \mathcal{D}_{1}\right)$

From our previous results, we easily deduce the following:

Theorem 3 (Democracy) Assume that $|\mathcal{Z}| \geq 3$. For any social rule $\Gamma$ that satisfies:

- unlimited domain or unlimited maximal domain,

- independence of irrelevant alternatives,

- weak Pareto,

- anonymity,

there exists a unique oligarchy, which is the whole society.

Note that if the whole society $\mathcal{H}$ is an oligarchy, this means that

$$
\cap_{i} \mathcal{D}_{i} \subseteq \Gamma\left(\left[\mathcal{D}_{i}\right]\right) \subseteq \cap_{i}\left(-\mathcal{D}_{i}\right)^{c}
$$

or, in other words, that $\Gamma$ satisfies weak Pareto and that $f \in \cup_{i} \mathcal{D}_{i}$, then $-f$ cannot belong to $\Gamma\left(\left[\mathcal{D}_{i}\right]\right)$. Thus, the social rule cannot contradict any of the individual choices of the voters, and must incorporate those options where all of them agree. Note also that, considering the comments in the previous section, democracy is incompatible with the assumption of completeness.

Conversely, it is not difficult to show that democracy is equivalent to anonymity, provided the other axioms in Theorem 2 are satisfied:

Proposition 3 Assume that $|\mathcal{Z}| \geq 3$. For any social rule $\Gamma$ that satisfies:

- unlimited domain or unlimited maximal domain,

- independence of irrelevant alternatives,

- weak Pareto,

if the whole society is an oligarchy, then $\Gamma$ satisfies anonymity.

To see that this does not hold when the social rule does not satisfy independence of irrelevant alternatives, it suffices to modify slightly Example 2: if we take $n=2$ and define

$$
\Gamma\left(\left[\mathcal{D}_{1}, \mathcal{D}_{2}\right]\right)=\left(\mathcal{D}_{1} \cap \mathcal{D}_{2}\right) \cup\left(\mathcal{D}_{1 \triangleright} \cap\left(-\mathcal{D}_{2}\right)^{c}\right),
$$

we deduce that $\mathcal{G}=\{1,2\}$ is an oligarchy (i.e., $\Gamma$ is a democracy), although $\Gamma\left(\left[\mathcal{D}_{1}, \mathcal{D}_{2}\right]\right) \neq$ $\Gamma\left(\left[\mathcal{D}_{2}, \mathcal{D}_{1}\right]\right)$ : if $\mathcal{D}_{2}=\mathcal{L}^{+}$, we obtain

$$
\Gamma\left(\left[\mathcal{D}_{1}, \mathcal{D}_{2}\right]\right)=\mathcal{D}_{1 \triangleright} \text { and } \Gamma\left(\left[\mathcal{D}_{2}, \mathcal{D}_{1}\right]\right)=\mathcal{L}^{+}
$$




\section{Coherent social rules}

So far we have studied how desirability can model social choice and detailed the specificities of the latter in the desirability context. Now we study the relation between social choice and desirability at a more fundamental level by focusing on coherence. Our aim is to understand what are the implications of coherence on social choice.

To this end, we start by representing profiles in the joint space $\mathcal{H} \times \mathcal{Z}$ of voters and their opinions: it is enough to extend the generic gamble $f_{i} \in \mathcal{D}_{i}$ into the gamble on $\mathcal{H} \times \mathcal{Z}$ that is equal to $f_{i}$ when we consider voter $i$ and is equal to zero otherwise. In other words, $f$ is a gamble conditional on considering voter $i$. We call $\mathcal{D} \mid i$ the representation of $\mathcal{D}_{i}$ in the space $\mathcal{H} \times \mathcal{Z}$ obtained by taking the (conditional) extension of the gambles in $\mathcal{D}_{i}$. At this point we can take the union of $\mathcal{D} \mid i$ for all voters $i$; this allows us to talk of all voters and opinions together, without having introduced any new information. Finally we call $\mathcal{E}$ the natural extension of the union. Let us recall that the operation of natural extension is essentially a deductive closure that computes all the 'logical' implications of some given assessments, which in the present case are the voters' opinions.

The next lemma shows that $\mathcal{E}$ is automatically coherent and that its marginal is given by $\cap_{i \in \mathcal{H}} \mathcal{D}_{i}$ :

Lemma 4 Consider a set of voters $\mathcal{H}$ and a profile $\left[\mathcal{D}_{i}\right]$. For each $i \in \mathcal{H}$, let us define $\mathcal{D} \mid i$ on the product space $\mathcal{H} \times \mathcal{Z}$ by:

$$
\mathcal{D} \mid i:=\left\{f \in \mathcal{L}(\mathcal{H} \times \mathcal{Z}): f=\mathbb{I}_{i} \otimes f_{i}, f_{i} \in \mathcal{D}_{i}\right\},
$$

where $\mathbb{I}_{i} \otimes f_{i}$ is the gamble given by

$$
\mathbb{I}_{i} \otimes f_{i}(j, z):= \begin{cases}f_{i}(z) & \text { if } j=i \\ 0 & \text { otherwise. }\end{cases}
$$

Then $\cap_{i \in \mathcal{H}} \mathcal{D}_{i}=\operatorname{Marg}_{\mathcal{Z}}(\mathcal{E})$, where

$$
\mathcal{E}:=\operatorname{posi}\left(\cup_{i \in \mathcal{H}}(\mathcal{D} \mid i) \cup \mathcal{L}^{+}(\mathcal{H} \times \mathcal{Z})\right)
$$

is a coherent set and $\operatorname{Marg}_{\mathcal{Z}}(\mathcal{E})$ is given by Definition 9 .

That the natural extension is coherent implies that we can always merge the voters' opinions into a compromise that is logically consistent with those opinions. In addition, the fact that the voters' shared viewpoints, represented by the set $\cap_{i \in \mathcal{H}} \mathcal{D}_{i}$, coincide with the marginal of $\mathcal{E}$ on $\mathcal{Z}$, implies that at the very least the compromise will have to be implemented via weak Pareto. Saying this differently: if voters were not available even in the worst of cases to accept weak Pareto as a social rule, then any social rule would have to be logically inconsistent with some of the originally expressed opinions. ${ }^{4}$

Under this light, weak Pareto turns out to be the last frontier before incoherence. This suggests the following definition:

Definition 26 (Coherent social rule) A coherent social rule is a social rule such that for every profile $\left[\mathcal{D}_{i}\right] \in \mathcal{A}$,

where $\mathcal{E}$ is defined as in (6).

$$
\Gamma\left(\left[\mathcal{D}_{i}\right]\right) \supseteq \operatorname{Marg}_{\mathcal{Z}}(\mathcal{E}),
$$

\footnotetext{
${ }^{4}$ While there is quite a general consensus on the acceptability of weak Pareto, it has also received some criticism; see for instance $[18,36]$.
} 
The next theorem show that such a definition is justified as weak Pareto is not only necessary for keeping together the voters' opinions coherently, but also sufficient.

Theorem 4 Let $\Gamma$ be a social rule. Consider a set of voters $\mathcal{H}$ with profile $\left[\mathcal{D}_{i}\right]$, and let $\mathcal{D} \mid i$ be given by (5) for every $i \in \mathcal{H}$. Let us define also

$$
\mathcal{E}:=\left\{\sum_{i \in \mathcal{H}} \mathbb{I}_{i} \otimes f_{i}:(\forall i \in \mathcal{H}) f_{i} \in \mathcal{D}_{i} \cup\{0\}\right\} \backslash\{0\} .
$$

Then

1. $\mathcal{E}$ is equal to the set in (6).

2. $\Gamma\left(\left[\mathcal{D}_{i}\right]\right) \supseteq \operatorname{Marg}_{\mathcal{Z}}(\mathcal{E})$ is equivalent to the existence of a coherent $\mathcal{E}^{\prime} \supseteq \mathcal{E}$ such that $\Gamma\left(\left[\mathcal{D}_{i}\right]\right)=\operatorname{Marg}_{\mathcal{Z}}\left(\mathcal{E}^{\prime}\right)$.

3. The smallest such set is

$$
\mathcal{E}^{\prime}:=\left\{f_{0} \otimes \mathcal{H}+\sum_{i \in \mathcal{H}} \mathbb{I}_{i} \otimes f_{i}: f_{0} \in \Gamma\left(\left[\mathcal{D}_{i}\right]\right) \cup\{0\},(\forall i \in \mathcal{H}) f_{i} \in \mathcal{D}_{i} \cup\{0\}\right\} \backslash\{0\} .
$$

4. For all $i \in \mathcal{H}, \mathcal{E}^{\prime}|i=\mathcal{D}| i$.

5. $\operatorname{Marg}_{\mathcal{H}}\left(\mathcal{E}^{\prime}\right)=\mathcal{L}^{+}(\mathcal{H}) \cup\left\{f \in \mathcal{L}(\mathcal{H}):\left(\exists f_{0} \in \Gamma\left(\left[\mathcal{D}_{i}\right]\right)\right) f \geq \bar{P}\left(f_{0} \mid \mathcal{H}\right)\right\}$, where the inequality is meant to be strict on all those $i$ such that $\bar{P}\left(f_{0} \mid i\right)-f_{0} \notin \mathcal{D}_{i}$.

In particular, point 2 in the theorem shows that weak Pareto is equivalent to the existence of a social rule that is logically consistent (coherent) with the voters' opinions. Rather than regarding this as a justification for weak Pareto-which seems to be easily acceptable as an axiom-, we understand this result as a way to highlight an important feature of weak Pareto, i.e., its being the 'guardian' of the coherence of a social rule with the voters' opinions: all and only coherent social rules are those that contain $\cap_{i \in \mathcal{H}} \mathcal{D}_{i}$.

In points 3-5, we detail some characteristics of the least-committal set $\mathcal{E}^{\prime}$, in the space $\mathcal{H} \times \mathcal{Z}$, associated with a coherent social rule: point 3 details its form; point 4 reassures us that $\mathcal{E}^{\prime}$ yields the same conditionals from which we started; point 5 reports the shape of its marginal over $\mathcal{H}$, i.e., the set that describes the relative importance (weight) of the voters in the definition of the social rule.

\section{Complete coherent social rules and state independence}

We can get a deeper understanding of coherent social rules by paying closer attention to the special case of complete social rules that are defined on linear previsions; namely, those for which $\Gamma\left(\left[P_{1}, \ldots, P_{n}\right]\right)=P$, for a certain linear prevision $P$ (remember the correspondences in Section 2 between desirable gambles and linear previsions, in particular Definitions 11 and 14; they allow us to talk of social rules in terms of traditional probability):

Corollary 1 Let $\Gamma$ be a social rule that assigns, to any profile $\left[P_{i}\right]$ of linear previsions, another linear prevision $P$. If $\Gamma$ satisfies weak Pareto, then for any profile $\left[P_{i}\right]$ there exists a probability mass function $\pi$ over $\{1, \ldots, n\}$ such that $P=\sum_{i} \pi(i) P_{i}$.

Example 3 As a simple illustrative example, consider a set of $n=3$ voters, a possibility space $\mathcal{S}$ and a set of prizes $\mathcal{X}$, both with cardinality two. For simplicity, we will indicate with 
$s_{1}, s_{2}$ the elements of $\mathcal{S}, x_{1}, x_{2}$ the elements of $\mathcal{X}$ and $z_{1}:=\left(s_{1}, x_{1}\right), z_{2}:=\left(s_{1}, x_{2}\right), z_{3}:=$ $\left(s_{2}, x_{1}\right), z_{4}:=\left(s_{2}, x_{2}\right)$ the elements of $\mathcal{Z}=\mathcal{S} \times \mathcal{X}$.

Suppose furthermore that the opinions of each voter $i$ can be always expressed through a linear prevision $P_{i}$ on $\mathcal{L}(\mathcal{Z})$ or equivalently through a maximally strictly desirable set of gambles $\mathcal{D}_{i, \triangleright}$. Given the fact that each linear prevision is in one-to-one correspondence with a finitely additive probability, which can be obtained by making the restriction of linear previsions to indicators of events, in what follows we will work directly with those probabilities, specifying their values only on the elementary events.

Corollary 1 guarantees that every social rule $\Gamma$ that assigns to every profile of opinions (i.e., every profile of linear previsions) another linear prevision $P$ and satisfies weak Pareto, satisfies also $P=\sum_{i} \pi(i) P_{i}$, where $\pi$ is a probability mass function over $\{1, \ldots, n\}$. Let us indicate, in the context of this example, a social rule that satisfies the hypothesis of Corollary 1 with the term admissible.

This means in particular that, if we consider the profile $\left[P_{i}\right]$, where:

$$
P_{i}\left(z_{i}\right)=1, i \in\{1,2\}, P_{3}=(1 / 3,0,2 / 3,0),
$$

that is equivalent to a profile of maximally strictly desirable sets of gambles $\left[\mathcal{D}_{i, \triangleright}\right]$, where:

$$
\begin{aligned}
& \mathcal{D}_{i, \triangleright}=\mathcal{L}^{+} \cup\left\{f \in \mathcal{L}: f\left(z_{i}\right)>0\right\}, i \in\{1,2\}, \text { and } \\
& \mathcal{D}_{3, \triangleright}=\mathcal{L}^{+} \cup\left\{f \in \mathcal{L}: \frac{1}{3} f\left(z_{1}\right)+\frac{2}{3} f\left(z_{3}\right)>0\right\},
\end{aligned}
$$

every (and only) weighted average of $P_{i}$, i.e., every element of the convex hull of $\left\{P_{1}, P_{2}, P_{3}\right\}$, constitutes the outcome of an admissible social rule.

Figure 1 gives a graphical representation of this set of probabilities (the grey filled polytope), within the simplex spanned by the degenerate probability measures $P_{z_{1}}:=P_{1}=$ $(1,0,0,0), P_{z_{2}}:=P_{2}=(0,1,0,0)$ and $P_{z_{3}}:=(0,0,1,0)$ (we use a three-dimensional simplex by assuming that the probability of $z_{4}$ is equal to 0 ).

To prove that there are linear previsions that cannot constitute the outcome of an admissible social rule for this profile, consider the linear prevision associated with $P_{z_{3}}$. This linear prevision is incompatible with the assumption of weak Pareto: in fact, note for instance that the gamble $f=(3,3,-1,-1)$ is strictly desirable for $P_{1}, P_{2}, P_{3}$ but not for $P_{z_{3}}$ (i.e., it is contained in the strictly desirable set of gambles associated with $P_{1}, P_{2}, P_{3}$, but not in the one associated with $\left.P_{z_{3}}\right)$. $\diamond$

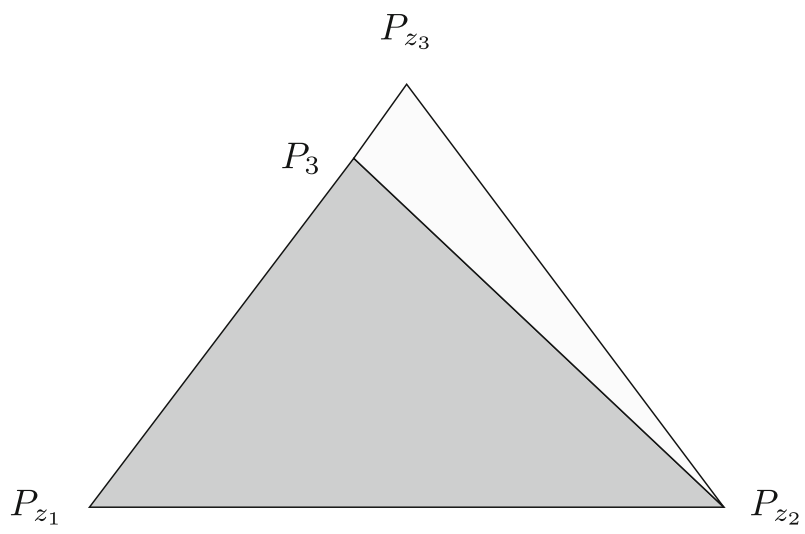

Fig. 1 Credal set of Example 3 
This corollary portrays a picture of coherent social rules that is not obvious to see from the general case of Theorem 4: it shows that in the complete case coherent social rules turn out to be just mixtures of the original linear previsions. In other words, Corollary 1 places coherent social rules, in the precise case, very close to linear opinion pooling; the main difference being that in linear pooling the experts' weights are traditionally fixed while in coherent social rules they can change with the profile (see also [53, Theorem 6.2]). ${ }^{5}$ However both are based on the idea of pooling opinions by taking convex combinations, i.e., by a linear approach to pooling. ${ }^{6}$

As an application of Corollary 1, we show next that eliminating the hypothesis of independence of irrelevant alternatives while adding some other conditions, we can find again a dictatorship where, in particular, the dictator determines completely the social rule (for a similar version of the following theorems using preference profiles see [46]).

To this end, we need to explicitly distinguish voters' probabilities from utilities, ${ }^{7}$ and to allow them to factorise:

Definition 27 (State independence) A probability measure $P$ on $\mathcal{S} \times \mathcal{X}$ is state independent if and only if possibilities and prizes are stochastically independent. A complete social rule $\Gamma$ satisfies state independence if and only if for each profile $\left[P_{i}\right]$, the probability measure $\Gamma\left(\left[P_{i}\right]\right)$ satisfies state independence. It is said to have state independent domain if and only if for each profile $\left[P_{i}\right]$ in its domain, $P_{i}$ is a state independent probability measure for every $i$.

Let us recall that state independence is the standard assumption that allows us to separately talk of probabilities and utilities, and as such it has broad implications in the literature for the theory and applications of decision making.

We have seen that a social rule that satisfies the hypotheses of Corollary 1 always induces a linear prevision that is a linear pooling with weights that depend on the profile. In the more traditional case in which weights are constant with respect to the profile, we have the following:

Theorem 5 Let $\Gamma$ be a social rule. If $\Gamma$ satisfies:

$\begin{array}{ll}- & \text { state independent domain, } \\ \circ & \text { weak Pareto, } \\ \circ \quad \text { completeness, } \\ \circ \quad \text { state independence, }\end{array}$

and if then there is a probability mass function $\pi$ on $\mathcal{H}$ such that for all $\left[\hat{\mathcal{D}}_{i}\right] \in \mathcal{A}$ the linear prevision induced by $\Gamma\left(\left[P_{i}\right]\right)$ on $\mathcal{Z}$ can be written as $\sum_{i \in \mathcal{H}} \pi(i) P_{i}$, then there exists $j \in \mathcal{H}$ such that $\pi(j)=1$.

\footnotetext{
${ }^{5}$ Let us recall that 'opinion' pooling should be understood in a broad sense in this paper, since our sets of desirable gambles, as well as lower previsions, model not only beliefs (opinions-probabilities) but also values (utilities) at the same time.

${ }^{6}$ Note that linear pooling can be subject to some specific criticism with regard to not preserving unanimous judgments of independence by the voters, relying on observations made in [28, 29], for instance. Such a criticism does not apply to the imprecise case, though, as discussed in [48, Section 5] as well as later on in this paper, in Section 8.3.

${ }^{7}$ Also in this case, remember that it is Definitions 11 and 14 that allow us to talk of social rules in terms of traditional probability (and utility).
} 
The theorem states in particular that, if we want to preserve state independence, we must have a degenerate distribution. To see an example, consider a possibility space $\mathcal{S}$ and a set of prizes $\mathcal{X}$ of cardinality two as in Example 3 . You can notice that the social rule $\Gamma$ defined as $\Gamma\left(\left[P_{1}, P_{2}, P_{3}\right]\right)=\frac{1}{3} P_{1}+\frac{1}{3} P_{2}+\frac{1}{3} P_{3}$ for every profile $\left[P_{i}\right]$ of linear previsions, does not satisfy state independence even if $\left[P_{i}\right]$ is a profile of state independent probability measures (consider for instance the profile $\left[P_{i}\right]$ analysed in Example 3). Instead, the social rule $\Gamma^{\prime}$ defined as $\Gamma^{\prime}\left(\left[P_{1}, P_{2}, P_{3}\right]\right)=P_{1}$ for every profile of state independent probability measures, satisfies state independence.

In the proof of the theorem, we deduce something different with respect to [46]: that $\pi$ (independent of the profile) is degenerate, meaning that it is always the same individual that determines the collective choices. In other words, we obtain a dictatorship with precise degenerates.

Note that in the general case in which weights depend on the profile, the theorem is not valid even if we ask only for state independence. Denote by $\left(P_{i}, U_{i}\right)$ the state dependent preferences of voter $i$, and consider the social rule $\Gamma\left(\left[\left(P_{1}, U_{1}\right), \ldots,\left(P_{n}, U_{n}\right)\right]\right)$ given by

$$
\begin{cases}\left(P_{1},\left(U_{1}+\cdots+U_{n}\right) / n\right) & \text { if } P_{1}=\cdots=P_{n} \\ \left(\left(P_{1}+\cdots+P_{n}\right) / n, U_{1}\right) & \text { if } U_{1}=\cdots=U_{n} \\ \left(P_{1}, U_{1}\right) & \text { otherwise; }\end{cases}
$$

this rule satisfies independence even if it is not a dictatorship. A detailed study of this problem can be found in [24].

This nevertheless, the assumption of fixed weights, albeit somewhat restrictive, is commonplace when the social rule corresponds to a weighted average of the different elements of the profiles, and the weights are determined by the relative importance of the voters (experts); see [53, Section 6.2] and [44, 49] for some comments in this respect. Our result shows that such a rule is only compatible with a dictatorship in the case of state independence. Interestingly, the portrayed situation reminds Bayesian model averaging, where a certain model is obtained as a mixture of experts; indeed a characteristic of model averaging is that it peaks around a single expert with more data, as if only one of the experts should always be the 'right' one in the limit. This behaviour, not always desirable (consider for instance the success of ensemble models in machine learning), seems to point to the fact that the limit behaviour of model averaging complies with Theorem 5. This appears to hint at either relaxing the assumption of precision or that of state independence.

It is still an open problem at this stage whether there are sufficient conditions for an autocracy: i.e., that $\pi$ is degenerate for each profile, even if it is not always degenerate on the same voter, like in a dictatorship.

Additional discussion of the relation between coherent social rules and independence, understood in a broad sense, is reported in Section 8.3.

Finally, let us remark that linearity of opinion pooling in complete coherent social rules somewhat reverberates also in the more general incomplete case. In fact, since $\mathcal{E}^{\prime}$ in (7) is coherent, it is the intersection of a number of maximal coherent sets of desirable gambles; these being precise models, we could develop for them a result similar to Corollary1; whence $\mathcal{E}^{\prime}$ could be represented as the intersection of a number of linear pooling models. For example, in a special case, it would become a linear pooling, with imprecise weights, of sets of linear previsions; this includes as a further special case what Stewart and Ojea Quintana call 'convex opinion pooling' [48, Section 5]. 


\section{An illustrative example}

As we have mentioned, one of the advantages of sets of desirable gambles is that they can be employed both in the problem of finding collective preferences in a voting context and in that of aggregating subjective beliefs and values. In addition, they allow us to deal with incomplete information and also to distinguish between indifference and incomparability. In this section, we shall illustrate these features and the main results in the paper by means of an example. We report also the following diagram as a quick reference to the main results obtained in this paper, with their respective theorems (Fig. 2).

Consider a group of four friends (Alice, Bob, Carol and David) that are discussing their plans for the upcoming weekend. They must choose between several options, whose interest for the group is influenced by the weather. Assume that they consider three possible states $\Omega:=\{s, c, r\}$, which are abbreviations of 'sunny', 'cloudy' and 'rainy'. Then each of them may model their subjective beliefs about the weather by means of a (possibly imprecise) uncertainty model. To illustrate the several possibilities that have appeared along this paper, we consider the following:

- Alice is certain that the weekend will be sunny. She expresses this with the degenerate probability $P_{A}$ for which $P_{A}(s)=1$.

- Bob is less certain about the weather but is also sure that it will not rain. He expresses it by means of the assessment $P_{B}(\{s, c\})=1$, which leads to the credal set $\mathcal{M}_{B}:=$ $\{P: P(\{s, c\})=1\}$ or equivalently, to the lower prevision $\underline{P}$ that is vacuous on the set $\{s, c\}$, as given by $\underline{P}_{B}(f):=\min \{f(s), f(c)\}$ for every gamble $f$ on $\Omega$.

- Carol cannot model directly her beliefs in terms of a set of probability measures, but can nonetheless ascertain her preferences between certain gambles: specifically, if she must choose between a picnic in the park (gamble $f$ ) and a tennis match outdoor (gamble $g$ ), that the group models with the following rewards (in a given linear utility scale):

$$
f(\omega):=\left\{\begin{array}{ll}
2 & \text { if } \omega=s \\
1 & \text { if } \omega=c \\
-3 & \text { otherwise, }
\end{array} \text { and } g(\omega):= \begin{cases}1 & \text { if } \omega=s \\
2 & \text { if } \omega=c \\
-3 & \text { otherwise }\end{cases}\right.
$$

then the result is that she prefers $f$ to $g$. This is equivalent to the desirability of the gamble $f-g=\mathbb{I}_{s}-\mathbb{I}_{c}$, which in practice means that she considers more probable a sunny weekend than a cloudy one.

- David is completely unsure about all the options. He therefore regards any probability function over $\Omega$ as possible, and as a consequence he can only say that a gamble $f$ is preferable to a gamble $g$ when $f$ dominates $g$, in the sense that $f-g \in \mathcal{L}^{+}$.

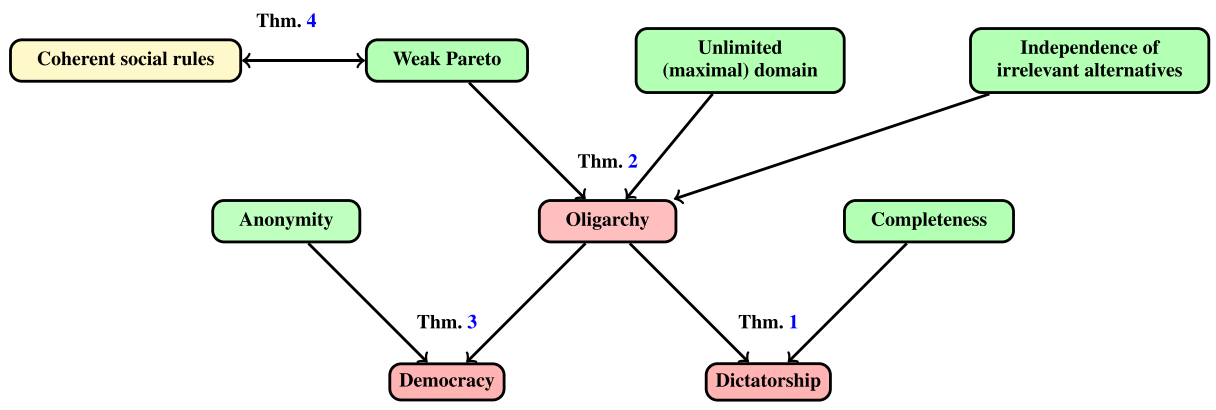

Fig. 2 A graphical summary of the results in the paper 
We can associate respectively with each friend the following coherent sets of desirable gambles:

○ $\mathcal{D}_{A}:=\mathcal{L}^{+} \cup\{f \in \mathcal{L}: f(s)>0\}$. It derives directly from (3) applied to $P_{A}$;

○ $\mathcal{D}_{B}:=\mathcal{L}^{+} \cup\{f \in \mathcal{L}: \max \{f(s), f(c)\}>0, \min \{f(s), f(c)\} \geq 0\}$. This follows applying (3) to $\underline{P}_{B}$;

○ $\mathcal{D}_{C}:=\operatorname{posi}\left(\left\{\mathbb{I}_{S}-\mathbb{I}_{C}\right\} \cup \mathcal{L}^{+}\right)$, the natural extension of $\mathbb{I}_{S}-\mathbb{I}_{C}$;

○ $\mathcal{D}_{D}:=\mathcal{L}^{+}$.

Now, in order to create a social rule that sensibly aggregates the four friends' opinions, we require it to satisfy the following properties:

- weak Pareto, as we regard it normative because it amounts to the formulation of coherence in the context of social choice (Definition 26): it means that those choices that are desirable for all of them are desirable for the group;

- unlimited domain, meaning that we place no restrictions on the set of desirable gambles that each friend may consider, nor any constraints on the relationships between the four; - independence of irrelevant alternatives, so that the desirability of an alternative for the group does not depend on its members' degree of acceptability: that is to say that under IIA, opinions, other than bare votes, do not matter.

Under these conditions, our Theorem 2 tells us that we must have an oligarchy.

If this oligarchy is made of the four friends (i.e., if it is a democracy), then we deduce from Proposition 2 that it must be $\Gamma_{\{A, B, C, D\}}\left(\left[\mathcal{D}_{i}\right]\right)=\cap_{i} \mathcal{D}_{i}=\mathcal{L}^{+}$. In this case, David's extreme caution forces the group to become vacuous, too. The same happens for any oligarchy that includes David. On the other hand, it is possible to show that the oligarchy of Alice and Carol produces $\Gamma_{\{A, C\}}\left(\left[\mathcal{D}_{i}\right]\right)=\mathcal{D}_{C}$, while that of Bob and Carol (and as a consequence also if we include Alice) gives $\Gamma_{\{B, C\}}\left(\left[\mathcal{D}_{i}\right]\right)=\mathcal{L}^{+}$.

If instead we consider an oligarchy made of Alice and Bob, we obtain that

$$
\Gamma_{\{A, B\}}\left(\left[\mathcal{D}_{i}\right]\right)=\mathcal{L}^{+} \cup\{f \in \mathcal{L}: f(s)>0, f(c) \geq 0\} .
$$

This is very similar to Bob's assessment, but we are excluding for instance gambles such as $(h(s), h(c), h(r))=(0,1,-1)$.

With this procedure we can also determine what is preferable to do for the group in some situations. Assume for instance that the choice of the group depends only on Alice and Bob, and that the two alternatives for the weekend are either attending an indoor party of spending the night in a cabin in the woods after a long walk outside. If we represent these two alternatives by means of the respective gambles $h$ and $t$ :

$$
h(\omega):=\left\{\begin{array}{ll}
1 & \text { if } \omega=s \\
2 & \text { if } \omega=c \\
0 & \text { otherwise, }
\end{array} \text { and } t(\omega):= \begin{cases}3 & \text { if } \omega=s \\
2 & \text { if } \omega=c \\
-2 & \text { otherwise, }\end{cases}\right.
$$

we conclude that the group will choose the cabin in the woods, because $t-h \in \Gamma_{\{A, B\}}\left(\left[\mathcal{D}_{i}\right]\right)$.

This also illustrates the advantage of working with sets of desirable gambles: if we consider the coherent lower prevision $\underline{P}_{A, B}$ associated with $\mathcal{D}_{1} \cap \mathcal{D}_{2}$, we obtain $\underline{P}_{A, B}(t-h)=$ 0 : that is, the gamble $t-h$ is desirable but not strictly desirable, whence its acceptability cannot be deduced from the coherent lower prevision. 


\section{Related work}

Our work in this paper has connections both with social choice and belief aggregation or opinion pooling. In this section, we discuss three main earlier approaches on these matters that appear to have tighter relations than others to the present paper.

\subsection{Pini, Rossi, Venable and Walsh on social choice}

We begin by discussing social choice theory. Since the publication of Arrow's theorem [3], there has been an extensive amount of work devoted to the analysis and generalisation of this result; see for instance $[26,56]$. These works assume that each of the voters establishes a (possibly incomplete) order between the outcomes, and that these should be aggregated into a global order.

We find of particular interest the work done by Pini, Rossi, Venable and Walsh (PRVW) in [43] on the aggregation of imprecise preferences (other relevant works dealing with incomplete orders over the outcomes can be found in $[6,20,25])$. Similarly to what is done in this paper, they consider incomplete orders, so that some alternatives can be deemed incomparable without being indifferent. One difference with our approach, though, is that they employ the classical Arrowian formalism of preferences over alternatives. Nevertheless, it is easy to establish an equivalence between preference relations and sets of desirable gambles, using

$$
f \succ g \Leftrightarrow f-g \in \mathcal{D}
$$

see [54, Section 3.7] for more details and [61] for a broader view encompassing utility considerations. In this respect, strict preferences, as above, give rise to coherent sets of desirable gambles as those used in this paper; weak preferences can be modelled similarly through axioms D2, D3, D10, D11 in [54, Appendix F] - the main difference being that the zero gamble must be included in a coherent set (see also [60, Section 6.2]).

An interesting feature of PRVW's approach is that they specify three different notions of dictator: ${ }^{8}$ strong dictator, whose preferences correspond to the aggregated ones in every profile, dictator, that corresponds to the notion we have given in Definition 23 and, finally, weak dictator who is simply an individual whose preferences in every profile are not contradicted by the aggregated ones. If we express these notions in our formalism we obtain the following definition:

Definition 28 (PRVW's dictator notions) Let $\Gamma$ be a social rule. An individual $i \in \mathcal{H}$ is called:

○ $\quad$ a strong dictator iff $\left(\forall\left[\mathcal{D}_{i}\right] \in \mathcal{A}\right) \Gamma\left(\left[\mathcal{D}_{i}\right]\right)=\mathcal{D}_{i}$;

- a dictator iff $\{i\}$ is decisive;

○ a weak dictator iff $(\forall f \in \mathcal{L})\left(\forall\left[\mathcal{D}_{i}\right] \in \mathcal{A}\right) f \in \mathcal{D}_{i} \Rightarrow-f \notin \Gamma\left(\left[\mathcal{D}_{i}\right]\right)$.

It is easy to see that

$$
\text { strong dictator } \Rightarrow \text { dictator } \Rightarrow \text { weak dictator, }
$$

and that the converses do not hold (although they do under completeness). This also shows that condition $\mathrm{O} 2$ of an oligarchy is a consequence of $\mathrm{O} 1$ when the oligarchy is made of only one voter.

\footnotetext{
${ }^{8}$ For other, slightly different notions of dictatorship, we refer to [27, 32].
} 
Using our results, we can give sufficient conditions for the existence of a strong or weak dictator:

Corollary 2 Assume that $|\mathcal{Z}| \geq 3$, and let $\Gamma$ be a social rule satisfying:

- independence of irrelevant alternatives,

- weak Pareto.

\section{Then:}

1. if $\Gamma$ satisfies unlimited domain or unlimited maximal domain, there exists at least one weak dictator;

2. if $\Gamma$ satisfies unlimited maximal domain and completeness, then there is a (unique) strong dictator $i \in \mathcal{H}$;

3. if $\Gamma$ satisfies anonymity and either unlimited domain or unlimited maximal domain, then any member of the society is a weak dictator.

It is interesting to note that our result is somewhat similar to [43, Theorem 5], with some important differences: the first item result does not require any further hypothesis on the social rule $\Gamma$ excepting the typical three ones of unlimited domain, independence of irrelevant alternatives and weak Pareto, whereas in [43, Theorem 5] it is assumed that the incomplete order determined by the social rule is restricted, meaning that all the top (resp., bottom) elements in the incomplete order it determines are indifferent to each other. It is also worth remarking that the idea that by not imposing completeness we can get rid of the Arrowian impossibility result is already present in that paper.

The difference between PRVW's conclusions and ours are likely due to the differences in the strength of the conditions imposed in the respective formulations of the problem. To make this clearer, consider a possibility space $\Omega$ made of three alternatives $\{a, b, c\}$. Then a complete preference relation on $\Omega$ such as $a \succ b \succ c$ would be translated into a coherent set of desirable gambles by stating that $\mathbb{I}_{a}-\mathbb{I}_{b}$ and $\mathbb{I}_{b}-\mathbb{I}_{c}$ are desirable, from which we derive, using natural extension, the coherent set of desirable gambles $\mathcal{D}:=\operatorname{posi}\left(\left\{\mathbb{I}_{a}-\mathbb{I}_{b}, \mathbb{I}_{b}-\right.\right.$ $\left.\left.\mathbb{I}_{c}\right\} \cup \mathcal{L}^{+}\right)$. However, this set of desirable gambles is not maximal, as we can tell if we notice that the credal set $\{P: P(a)>P(b)>P(c)\}$ has more than one element. This shows that our assumption of completeness (Definition 17) is stronger than the one that would be established on the preference relation on the alternatives.

Similarly, our assumption of independence of irrelevant alternatives (Definition 19) is also stronger than the one that is imposed in the standard setting of [43]. Indeed, while ours is requested for all gambles, the latter is requested only for those that correspond to a preference relation over the alternatives and we cannot derive the former by the latter. Indeed, if we consider a finite set of alternatives $\Omega$, it may be that a social rule satisfies unlimited domain, weak Pareto and independence of irrelevant alternatives only for the gambles of the type $\mathbb{I}_{\omega}-\mathbb{I}_{\omega^{\prime}}$ for $\omega \neq \omega^{\prime} \in \Omega$, while not satisfying it for all gambles.

Remark 2 These observations point to a more general question that seems useful to briefly discuss. Traditional work in social choice, such as PRVW's, does not establish a connection to probability, in the sense that the discussion begins and ends with preferences. In contrast, our preference-based formulation has a very natural probabilistic (as well as utility) counterpart, so that we can easily move back and forth between the two representations. This seems to be an advantage of our approach to social choice, and it is also what allows us to talk simultaneously of social choice and opinion pooling. On the other hand, this creates 
some dissimilarity between our work and traditional social choice, of the type illustrated by the examples above. In case one needs to stay closer to the traditional setting in social choice, then we foresee that two moves should be in order: the first is expressing preferences only on indicator functions of outcomes, as it is done above-and yet that is not enough as we have seen-; in fact, the second move is to force indicator functions of outcomes to be all and only the gambles one can deal with. This second step amounts to defining from the very beginning that the space of gambles is made only of indicator functions of outcomes, and their differences; and hence to stick throughout the work to the notion of a coherent set of gambles relative to a subset of $\mathcal{L}$, as we have done in a special case in Definition 8 . This should allow for keeping together the traditional view of social choice with a flexible probabilistic counterpart, as in this paper. $\diamond$

\subsection{Walley on belief aggregation}

Our work in this paper is also related to Walley's in [53] on the aggregation of imprecise beliefs (see also [54, Sections 4.37, 4.38]). In such a work, Walley discusses in detail a number of properties that an aggregation rule may or may not satisfy, and then analyses some aggregation rules in this respect. One major difference between our work and Walley's is that he is concerned with characterising a broad range of situations for the aggregation of beliefs; in this regard, we instead stay narrow trying to go deep in the attempt to characterise the least-committal normative property of a social rule, which, as we have seen, is its coherence with the given profile (Definition 26). We agree that in special cases a number of additional properties may be required besides coherence, but this type of analysis is not in the scope of the present paper.

According to those aims, Walley puts forward fifteen different axioms, while distinguishing between those that are in his opinion essential, from those that are desirable and those whose interest is doubtful, in his view. Below, we discuss these axioms in connection with the work carried out in this paper. Although in some cases the axioms are defined in terms of credal sets or coherent lower previsions, we shall give the definition in terms of sets of desirable gambles.

We begin by considering the axioms that according to Walley an aggregation procedure should satisfy:

Definition 29 (Walley's essential properties) A social rule $\Gamma$ is said to satisfy:

- coherence iff $\left(\forall\left[\mathcal{D}_{i}\right] \in \mathcal{A}\right) \Gamma\left(\left[\mathcal{D}_{i}\right]\right)$ is a coherent set of desirable gambles;

○ unanimity iff $\left(\forall\left[\mathcal{D}_{i}\right] \in \mathcal{A}\right) \cap_{i \in \mathcal{H}} \mathcal{D}_{i} \subseteq \Gamma\left(\left[\mathcal{D}_{i}\right]\right)$;

- reconciliation iff $\left(\forall\left[\mathcal{D}_{i}\right] \in \mathcal{A}\right) \Gamma\left(\left[\mathcal{D}_{i}\right]\right) \subseteq \cap_{i \in \mathcal{H}}\left(-\mathcal{D}_{i}\right)^{c}$;

○ indeterminacy iff $\left(\forall\left[\mathcal{D}_{i}\right] \in \mathcal{A}\right) \Gamma\left(\left[\mathcal{D}_{i}\right]\right) \subseteq\left\{\sum_{j} f_{j}: f_{j} \in \mathcal{D}_{j}\right\}$.

In our context, any social rule satisfies the property of coherence by Definition 16 . Unanimity corresponds to the property of weak Pareto, which we have justified in Theorem 4 as a rationality axiom that we actually call 'coherence of a social rule' (Definition 26).

The idea behind reconciliation is that the aggregated set should not be in direct conflict with the assessments of any of the voters. It can be equivalently expressed as saying that if $f \in \mathcal{D}_{i}$ for some $i$, then $-f$ cannot belong to $\Gamma\left(\left[\mathcal{D}_{i}\right]\right)$. Therefore, it corresponds to requiring that all the voters (experts) are weak dictators according to Definition 28, or, equivalently, to condition $\mathrm{O} 2$ in the definition of oligarchy, which in the case of reconciliation should correspond to the whole society. Thus, we believe that in some cases reconciliation 
may well be too restrictive. This is also illustrated because, as already remarked by Walley in [53], reconciliation forces the aggregated model to be imprecise when there is some conflict between the individual opinions. In fact, if we have unlimited maximal domain, reconciliation implies that we should have $\Gamma\left(\left[\mathcal{D}_{i}\right]\right) \subseteq \cap_{i} \mathcal{D}_{i}$. As a consequence, the only social rule satisfying reconciliation and unanimity would be given by $\Gamma\left(\left[\mathcal{D}_{i}\right]\right)=\cap_{i} \mathcal{D}_{i}$, in the case of unlimited maximal domain, which seems to be far too imprecise to be taken as a general solution of the aggregation problem even under the domain assumption. In fact, this question is related to our Proposition 2, where we have established a similar result under unlimited domain but where we ask in addition independence of irrelevant alternatives.

On the other hand, indeterminacy means that the coherent set $\Gamma\left(\left[\mathcal{D}_{i}\right]\right)$ should be included in the natural extension of $\cup_{i} \mathcal{D}_{i}$, that is, that any gamble that is considered desirable in the aggregated set should be a positive linear combination of gambles that are desirable for at least some voter. Although reasonable, this condition may be too uninformative in the case of conflicting opinions: if in a profile $\left[\mathcal{D}_{i}\right]$ there exist two elements $i_{1} \neq i_{2}$ and a gamble $f$ such that $f \in \mathcal{D}_{i_{1}}$ and $-f \in \mathcal{D}_{i_{2}}$, then it follows that $\left\{\sum_{j} f_{j}: f_{j} \in \mathcal{D}_{j}\right\}=\mathcal{L}$ and indeterminacy would be trivial. Since in the case of unlimited maximal domain there will be conflict unless all the sets in the profile coincide, in [53] it is considered the condition of strong indeterminacy, which can be extended to conflicting opinions, and which holds when $\Gamma\left(\left[\mathcal{D}_{i}\right]\right) \subseteq \cup_{i} \mathcal{D}_{i}$. It can be checked that this last condition follows from weak Pareto in the case of unlimited maximal domain: to see this, note that if $f \notin \cup_{i} \mathcal{D}_{i}$ then $-f \in \cap_{i} \mathcal{D}_{i} \subseteq$ $\Gamma\left(\left[\mathcal{D}_{i}\right]\right)$ and as a consequence of coherence $f \notin \Gamma\left(\left[\mathcal{D}_{i}\right]\right)$.

In summary, our view of the 'essential properties' for an aggregation rule differ from Walley's: we agree that his coherence and unanimity (weak Pareto) should be normative prescriptions. We do not, however, see room for granting a normative status to reconciliation and indeterminacy.

Next we discuss the conditions that Walley considers desirable in [53] for an aggregation rule. These are less relevant to our paper, given their non-essential status; yet it is interesting to see how they relate to our setting.

Definition 30 (Walley's desirable properties) A social rule $\Gamma$ is said to satisfy:

○ $\quad$ strong Pareto iff $\left(\forall\left[\mathcal{D}_{i}\right] \in \mathcal{A}\right) \Gamma\left(\left[\mathcal{D}_{i}\right]\right) \supseteq\left(\cap_{i \in \mathcal{H}}\left(-\mathcal{D}_{i}\right)^{c}\right) \cap\left(\cup_{i \in \mathcal{H}} \mathcal{D}_{i}\right)$;

- conjunction iff $\left(\forall\left[\mathcal{D}_{i}\right] \in \mathcal{A}\right) \Gamma\left(\left[\mathcal{D}_{i}\right]\right) \supseteq \operatorname{posi}\left(\cup_{i} \mathcal{D}_{i}\right)$ whenever the latter is coherent;

- complete ignorance iff $\Gamma\left(\left[\mathcal{D}_{i}\right]_{i \in \mathcal{H}}\right)=\Gamma\left(\left[\mathcal{D}_{i}\right]_{i \in \mathcal{H} \backslash\{j\}}\right)$ when $\mathcal{D}_{j}=\mathcal{L}^{+}$;

- relative ignorance iff $\Gamma\left(\left[\mathcal{D}_{i}\right]_{i \in \mathcal{H}}\right)=\Gamma\left(\left[\mathcal{D}_{i}\right]_{i \in \mathcal{H} \backslash\{j\}}\right)$ when $\mathcal{D}_{j}=\cup_{i \in \mathcal{H} \backslash\{j\}} \mathcal{D}_{i}$.

The rationale underlying strong Pareto is that a gamble that is desirable for some voter and not undesirable for any other voter (in the sense that no other voter considers $-f$ strictly desirable) should be included in the aggregated set. However, as already remarked by Walley, this condition is incompatible with that of coherence and unlimited domain. To see an example, let $\Omega:=\left\{\omega_{1}, \omega_{2}, \omega_{3}\right\}$ and consider the profile $\left[\mathcal{D}_{i}\right]_{i \in\{1,2,3\}}$ such that $\mathcal{D}_{i}:=\operatorname{posi}\left(\left\{f_{i}\right\} \cup \mathcal{L}^{+}(\Omega)\right)$, where

$$
f_{i}(\omega):= \begin{cases}-3 & \text { if } \omega=\omega_{i} \\ 1 & \text { otherwise }\end{cases}
$$

Clearly $f_{i} \in\left(\cap_{i \in \mathcal{H}}\left(-\mathcal{D}_{i}\right)^{c}\right) \cap\left(\cup_{i \in \mathcal{H}} \mathcal{D}_{i}\right)$ for all $\omega_{i} \in \Omega$. Hence if $\Gamma$ satisfies strong Pareto, $f_{i} \in \Gamma\left(\left[\mathcal{D}_{i}\right]\right)$ for all $\omega_{i} \in \Omega$. This means that $f_{1}+f_{2}+f_{3}=-1 \in \Gamma\left(\left[\mathcal{D}_{i}\right]\right)$ and so $\Gamma\left(\left[\mathcal{D}_{i}\right]\right)$ is not coherent.

The assumption of conjunction means that, if there is no conflict among the beliefs expressed by the voters, then the aggregated set should include the natural extension of the 
sets of gambles in the profile. It is trivial with the property of unlimited maximal domain, since, as we have already remarked, two maximal sets of gambles are in conflict unless they coincide. Conjunction is a converse of indeterminacy, so if the two of them hold together then we obtain that $\Gamma\left(\left[\mathcal{D}_{i}\right]\right)=\operatorname{posi}\left(\cup_{i} \mathcal{D}_{i}\right)$. It forces the join model to be at least as informative as all the individual models, while not necessarily being precise. Notice that thanks to our Proposition 2, we know that the property of conjunction is incompatible with the joint assumption of unlimited domain or unlimited maximal domain, independence of irrelevant alternatives and weak Pareto.

The notions of complete and relative ignorance refer to the consistency of a social rule defined on populations of different sizes, and say that if the beliefs of one voter are noninformative in absolute terms or with respect to the beliefs of the other voters, then they can be ignored as they have no implications over the aggregated set. Although interesting, the analysis of the consistency of the social rule as we vary the value of $n$ lies outside the scope of the present paper, and is one of the future lines of research.

Note also that Walley proposes a notion of strong reconciliation; considering our comments on the weaker notion of reconciliation we discussed earlier, we do not take this latter notion into account in this section. On the other hand, among the list of properties that a rule may or may not satisfy, he discusses a notion of symmetry, that corresponds to the notion of anonymity we have considered in Section 4.3.

Finally, we remark that among the criteria that Walley considers in [53], no one is equivalent to independence of irrelevant alternatives. As we discussed earlier, this condition implies that whether a gamble belongs or not to the aggregated set depends only on which elements of the profile that include $f$ and those that do not.

Walley's lack of discussion of such an assumption may be due to historical reasons, as the assumption is something natural and well known in the context of social choice, while it is less so when we consider a problem of belief aggregation. For instance, if we consider $n=2$ experts and the coherent lower previsions $\underline{P}_{1}, \underline{P}_{2}$ associated with $\mathcal{D}_{1}, \mathcal{D}_{2}$, it would mean that if a gamble $f$ satisfies $\underline{P}_{1}(f)=-0.1, \underline{P}_{2}(f)=100$ in one profile and $\underline{P}_{1}^{\prime}(f)=-100, \underline{P}_{2}^{\prime}(f)=0.1$ in another, it should be included in both aggregated sets or in neither of them. This is something that may not be reasonable in practice, because it forces us to effectively ignore the values that the gamble takes.

However, as we have seen in Section 7, independence of irrelevant alternatives may be a sensible assumption when we want to avoid considering the experts' opinions other than their acceptability assessments, so as to avoid, e.g., that stronger opinions are favoured. That the belonging to the aggregated set depends only on which elements of the profile include $f$, may also be sensible when we focus on gambles of a particular type, such as when we translate into the language of gambles incomplete preferences over the elements of a finite set. Alternatively, one could modify the assumption of independence of irrelevant alternatives into a form that may be more suited for belief aggregation, such as that proposed in [48] under the name of strong setwise function property.

Remark 3 We should also recall that other relevant works on the topic of aggregation of imprecise probabilities are those by Moral and del Sagrado [37] and by Nau [39]. The former extends works on linear or logarithmic pooling $[10,41]$ to the aggregation of credal sets determined by probability intervals, or coherent lower probabilities, using a credibility coefficient for each assessment. Nau considers two approaches based on confidence weights, so as to obtain something intermediate between the conjunction and the disjunction of the credal sets. $\diamond$ 


\subsection{Stewart and Ojea Quintana on opinion pooling}

Another interesting work that relates to ours is that by Stewart and Ojea Quintana (SOQ) in opinion pooling [48].

SOQ focus on the case where voters' profiles are maximally strictly desirable sets of gambles or, equivalently, vectors of (precise) probability functions. Yet, they allow for incompleteness of pooling operators under certain interpretations of pooling, in contrast to a tradition that takes pooling operators to be precise. In doing this, SOQ proceed by listing a number of desirable properties that a pooling operator should have, and eventually focus on 'convex opinion pooling' as an example that satisfies the desiderata. Convex opinion pooling is the idea of taking the convex hull of the initial probability functions.

As we mentioned already in Section 5, convex opinion pooling is a special case of coherent social rules, and hence it is compatible with the framework in this paper. In particular it is the special case that expresses total ignorance a priori about the relative importance of the voters' opinions. As such it can yield quite an imprecise group opinion.

One property that SOQ discuss is that of so-called probabilistic independence preservation. The rationale is that if all voters agree that two events are independent, then, reasonably, the social rule should regard them as independent too. As natural and innocuous as this observation may seem, it is unfortunately also something that cannot be achieved by linear opinion pooling, as it was pointed out long ago in related settings [28, 29]. This has obviously been detrimental to the very idea of linear pooling. On their side, SOQ prove that dictatorship is the social rule that satisfies both the properties of probabilistic independence preservation and the strong setwise function property. The latter is related to independence of irrelevant alternatives. However, they also show that when we let go of completeness by adopting convex opinion pooling, both requirements are met, thus solving an old debate with an imprecise twist.

Now we would like to leverage this observation by SOQ to illustrate quite broadly, by a simple argument, that in our setting the preservation of independence is not an issue when we let go of the axiom of completeness.

Assume first that each voter's preferences are modelled in terms of a coherent lower prevision $\underline{P}$, which in particular covers the case of precise probabilities. Then if $\left[\underline{P}_{i}\right]$ denotes our profile and $\underline{P}$ is the aggregated model, then we would say that the social rule preserves irrelevance of $A$ towards $B$ when

$$
(\forall i \in \mathcal{H}) \underline{P}_{i}(B \mid A)=\underline{P}_{i}(B) \Rightarrow \underline{P}(B \mid A)=\underline{P}(A) .
$$

More generally speaking, when the profile is modelled in terms of gambles, epistemic irrelevance of $A$ towards $B$ for the $i$-th voter means that

$$
(\forall \mu \in \mathbb{R}) \mathbb{I}_{A \cap B}-\mathbb{I}_{A} \mu \in \mathcal{D}_{i} \Leftrightarrow \mathbb{I}_{B}-\mu \in \mathcal{D}_{i},
$$

and the social rule would preserve independence when

$(\forall i \in \mathcal{H}) A$ epistemically irrelevant to $B \Rightarrow A$ epistemically irrelevant to $B$ in $\Gamma\left(\left[\mathcal{D}_{i}\right]\right)$.

Now, it is easy to see that (8) is preserved when taking lower envelopes: if we define $\Gamma\left(\left[\mathcal{D}_{i}\right]\right):=\cap_{i \in \mathcal{G}} \mathcal{D}_{i}$ for some $\mathcal{G}$, then for any pair of events $A, B$, we have that

$$
\begin{gathered}
{\left[(\forall \mu \in \mathbb{R}) \mathbb{I}_{A \cap B}-\mathbb{I}_{A} \mu \in \Gamma\left(\left[\mathcal{D}_{i}\right]\right) \Leftrightarrow \mathbb{I}_{B}-\mu \in \Gamma\left(\left[\mathcal{D}_{i}\right]\right)\right]} \\
\Leftrightarrow\left[(\forall \mu \in \mathbb{R}) \mathbb{I}_{A \cap B}-\mathbb{I}_{A} \mu \in \cap_{i \in \mathcal{G}} \mathcal{D}_{i} \Leftrightarrow \mathbb{I}_{B}-\mu \in \cap_{i \in \mathcal{G}} \mathcal{D}_{i}\right] \\
\Leftrightarrow\left[(\forall \mu \in \mathbb{R})(\forall i \in \mathcal{G}) \mathbb{I}_{A \cap B}-\mathbb{I}_{A} \mu \in \mathcal{D}_{i} \Leftrightarrow(\forall i \in \mathcal{G}) \mathbb{I}_{B}-\mu \in \mathcal{D}_{i}\right],
\end{gathered}
$$


and the last equivalence holds because of (8). Taking into account our results, this means that any oligarchy (and as a consequence any dictatorship, but also any democracy) would preserve independence.

\subsection{The work of Dietrich and List on social choice and opinion pooling}

We conclude this section by discussing the earlier work carried out on similar topics by Dietrich and List (DL), both in the context of social choice and of opinion pooling.

With respect to social choice, the most relevant papers for ours are [13, 14], which are both established in the language of propositional logic. ${ }^{9}$

In [13], DL show that, in a context of incompleteness, the aggregation of a number of consistent assessments leads to a dictatorship, provided some axioms (namely nonsimplicity, non-separability and accept-reject neutrality) hold. Thus, in [13] the authors establish a result similar to our Theorem 1. Nevertheless, there are certain differences: arguably the most important are that we are not imposing the neutrality condition in our results and we are considering coherent sets of desirable gambles that are the counterpart of not only consistent but also deductively closed set of propositions. Moreover, the definition of dictatorship is also different: it corresponds to what we called earlier a strong dictator.

On the other hand, in [14] sufficient conditions to the existence of a strong oligarchy are obtained, in terms of conditions of non-simplicity, non-affiness and path-connectedness; the latter is a closed variant of the total-blockness considered in [40]. Note that, for most of their results, there are also two conditions that we are not imposing, namely systematicity (that, in the language of this paper, means that whether a gamble $f$ belongs to $\Gamma\left[\mathcal{D}_{i}\right]$ depends only on the voters that consider $f$ desirable and the voters that do not consider it desirable, and not on the specific gamble) and monotonicity.

With respect to opinion pooling, the most relevant works are those in $[15,16]$. In [15], they characterise pooling functions that are linear and/or neutral (that in this paper means that the pooling function does not depend on the particular event, somewhat similarly to the systematicity axiom mentioned above), provided some assumptions of independence and unanimity hold. One of the keys in their results is that these assumptions are not imposed in the full domain, but only in some particular subclass satisfying some property of non-nestedness, non-simplicity or path-connectedness; by doing this, they generalise earlier results from [1,33]. Interestingly, in [15, Section 7] DL provide a connection with Arrow's theorem by considering the case of probabilistic preference aggregation. A somewhat similar point is made in [16], where the authors argue in detail that the assumptions of independence and unanimity should only be imposed on a subset of the $\sigma$-algebra, called the premises.

There are certain differences between the results by DL and those obtained in this paper: on the one hand, they focus on $\sigma$-additive probabilities on a $\sigma$-algebra of events, while we consider finitely additive probabilities. We are also more general in that we are only imposing weak Pareto in our Corollary 1, which implies unanimity but not independence; because of this, we are obtaining a weaker version of linear pooling, where the weights may depend on the profile. On the other hand, throughout this paper our rationality conditions are imposed in the whole domain of the aggregation rule, not on one subset.

\footnotetext{
${ }^{9}$ For the connection between propositional logic and the theory of coherent sets of desirable gambles, we refer to [7, 11]. In particular, the notion of consistency considered in [13] is related to avoiding partial loss, while deductive closure plus consistency is akin to coherence.
} 


\section{Conclusions}

Intuitively, it is quite clear that there is a common nature behind social choice and opinion pooling (belief aggregation). However, in the literature so far, the connection has apparently been explored only by Dietrich and List [15] in a special setting. This paper is an attempt to make the connection fully explicit by providing joint foundations of those fields through the formalism of sets of desirable gambles. Such a formalism appears particularly apt to the aim, since it allows us to move back and forth between probability-utility and preferences in a very natural way through duality $[60,61]$. Moreover, sets of desirable gambles empower us with very general modelling capabilities; they encompass as particular cases propositional logic, sets of probability measures, coherent lower previsions and non-additive measures, and moreover they are better suited for dealing with sets of measure zero [5, 55].

We have considered a problem of social choice (equivalently, of opinion pooling) where the subjects' joint states of beliefs and values (probabilities and utilities) are represented as coherent sets of desirable gambles. In addition to establishing a version of Arrow's theorem in this context, we have also shown that other forms of collective choice can be derived requiring different properties to satisfy for the social rule. In particular, we have shown that the weak Pareto assumption is tightly related to the coherence of a social rule, and that, roughly speaking, the only way to obtain a democracy preserving the properties considered in most of the literature about Arrowian framework extensions, is to eliminate the requirement of completeness and avoid conflicts, considering only opinions shared by the whole society.

Another reason for removing the assumption of completeness is that, as we have shown in Section 6, it is incompatible with the preservation of independence in the context of linear pooling, as already remarked in [29]; in contrast, we have shown in this paper that if we consider instead linear poolings where the aggregated model may be imprecise, we can preserve independence without incurring a dictatorship.

As we have mentioned, one advantage of working with sets of desirable gambles is that they allow us to connect our work with preference relations and with epistemic uncertainty. As a consequence, the results in this paper can be connected with those on social choice as well as with those on belief aggregation. This is not to say that any property required from one of these frameworks can readily be exported into the other: for instance, the assumption of independence of irrelevant alternatives appears to be particularly suited to model voting problems, in that it allows to neglect voters' opinions other than their bare votes; for the very same reason, such an assumption seems to be at odds with the idea of aggregating (imprecise) beliefs. Indeed, independence of irrelevant alternatives means that if a voter prefers an $f$ to another alternative $g$, for the joint decision it should not matter the strength of this preference: in the language of lower previsions, it may be that the gamble $f-g$ may be desirable because it has a lower prevision of 0.0001 or of 1000 , and both cases would be indistinguishable. If instead we want to exploit information of such a type, in order to take it into account into the decision, we can do it by weakening IIA and this would allow us to move away from the Arrowian theorems. This is not a new point: it was discussed as early as Nash [38] (see also [42] for an interesting work stemming from that paper).

In this vein, the unified approach we have considered in this paper should allow to investigate whether some axioms and results from one of the fields are sensible in the other. In this respect, in Section 8 we have shown how some of the desirable criteria in [53] for an aggregation rule are respected by our vision; how our results on oligarchy and democracy can be understood as an extension, in the context of desirability, of the ones of [43]; that 
the work in [48] can be regarded as an interesting special case of our proposal of coherent pooling operators; and the differences between our approach and the works carried out by Dietrich and List in $[13,15]$. In the future, it would be interesting to deepen the comparison with these other works, as well as other relevant studies in the literature, such as $[21,23$, 31], among others.

As another advantage of our unified approach, we can for instance mention that, taking into account the modelling of decision making using the theory of sets of desirable gambles in $[60,61]$, it should be a small step from this paper to make a similar modelling of multicriteria decision making, where the criteria take the role of the voters in our formulation of social choice. Some comments in this direction can be found in [17, 43].

With respect to other future developments, we envisage a few main avenues.

- Expanding on our Remark 2, in order to tighten even more the relation between traditional social choice and coherent sets of desirable gambles; this would allow traditional social choice researchers to enter a full probabilistic treatment of their area.

- Computational social choice is an active area of research $[9,45]$ that focuses on the algorithmic tasks in social choice and their complexity. An obvious path for future research would be to move the results in this paper closer to computational social choice. Another, less obvious, path, could be to modify the formulation of social choice in this paper to account for a limitation of computational resources directly at the level of the axioms of desirability. This would most likely yield a generalisation of social choice to quantum logic, since it has already been shown to be the case for desirability [8], thus opening up a number of interesting phenomena to investigate.

- With respect to desirable gambles, it would be interesting to study in more detail which properties from social choice can be extended to the modelling of preferences over sets of desirable gambles that not necessarily arise from binary comparisons of the alternatives, as in [51, 52].

Finally, it could be useful to extend our work to an infinite number of experts, or voters, which seems to be needed especially for statistical reasons. To this end, however, one should carefully deal with considerations of conglomerability in the case of desirable gambles [35, 59].

\section{Appendix A: Representation of a social rule $\Gamma$ as a bundle of functions}

A social rule $\Gamma$ can be represented as a bundle of functions $\left\{\Gamma_{f}: f \in \mathcal{L}\right\}$, where $\Gamma_{f}$ identifies those profiles for which the gamble $f$ belongs to the aggregated set

$$
\begin{aligned}
\Gamma_{f}: \mathbb{D}^{n} \rightarrow\{0,1\} \\
{\left[\mathcal{D}_{i}\right] \hookrightarrow \begin{cases}1 & \text { if } f \in \Gamma\left(\left[\mathcal{D}_{i}\right]\right) \\
0 & \text { otherwise. }\end{cases} }
\end{aligned}
$$

The fact that the social set of desirable gambles is coherent imposes some minimal requirements on these functions:

G1. $\Gamma_{\mathcal{L}^{+}}=1$.

G2. $\Gamma_{0}=0$.

G3. $f, g \in \mathcal{L} \Rightarrow \Gamma_{f+g} \geq \Gamma_{f} \cdot \Gamma_{g}$.

G4. $f \in \mathcal{L}, \lambda>0 \Rightarrow \Gamma_{f}=\Gamma_{\lambda f}$. 
We can reformulate additional properties that a social rule may satisfy using this equivalent representation. In particular:

- Completeness is equivalent to:

$$
f \neq 0 \Rightarrow \Gamma_{f}+\Gamma_{-f}=1,
$$

and strict completeness can be expressed as

$$
\varepsilon>0 \Rightarrow \Gamma_{f}+\Gamma_{-f+\varepsilon} \geq 1 \text {. }
$$

- Independence of irrelevant alternatives. Let us define the function

$$
\begin{gathered}
\pi_{f}: \mathbb{D}^{n} \rightarrow\{0,1\}^{n} \\
{\left[\mathcal{D}_{i}\right] \hookrightarrow \vec{z},}
\end{gathered}
$$

where

$$
\vec{z}_{i}:= \begin{cases}1 & \text { if } f \in \mathcal{D}_{i} \\ 0 & \text { otherwise }\end{cases}
$$

In other words, $\pi_{f}\left(\left[\mathcal{D}_{i}\right]\right)=\left(\mathbb{I}_{\mathcal{D}_{1}}(f), \ldots, \mathbb{I}_{\mathcal{D}_{n}}(f)\right)$. Then independence of irrelevant alternatives can be equivalently expressed as

$$
(\forall f \in \mathcal{L}) \pi_{f}\left(\left[\mathcal{D}_{i}\right]\right)=\pi_{f}\left(\left[\mathcal{D}_{i}^{\prime}\right]\right) \Rightarrow \Gamma_{f}\left(\left[\mathcal{D}_{i}\right]\right)=\Gamma_{f}\left(\left[\mathcal{D}_{i}^{\prime}\right]\right) .
$$

- Weak Pareto is equivalent to

$$
(\forall f \in \mathcal{L}) \pi_{f}^{-1}(\{\overrightarrow{1}\}) \subseteq \Gamma_{f}^{-1}(\{1\}) .
$$

- Given a set of individuals $\mathcal{G}$, it is almost decisive for $f$ if and only if

$$
\pi_{f}^{-1}\left(\overrightarrow{1}_{\mathcal{G}}, \overrightarrow{0}_{\mathcal{H} \backslash \mathcal{G}}\right) \subseteq \Gamma_{f}^{-1}(\{1\}),
$$

where $\overrightarrow{1}_{\mathcal{G}}, \overrightarrow{0}_{\mathcal{H} \backslash \mathcal{G}}$ is the vector $\vec{z}$ given by

$$
z_{i}:= \begin{cases}1 & \text { if } i \in \mathcal{G} \\ 0 & \text { if } i \notin \mathcal{G},\end{cases}
$$

whereas $\mathcal{G}$ is decisive for a gamble $f$ if and only if

$$
\pi_{f}^{-1}\left(\overrightarrow{1}_{\mathcal{G}}\right) \subseteq \Gamma_{f}^{-1}(\{1\}) .
$$

\section{Appendix B: Results in terms of lower previsions and sets of finitely additive probabilities}

In this paper, we have assumed that the beliefs of each voter are represented by means of a coherent set of desirable gambles $\mathcal{D}_{i}$, and that the outcome of the social rule $\Gamma$ on a profile $\left[\mathcal{D}_{i}\right]$ is also a coherent set of desirable gambles. The main motivation behind this choice lies in the flexibility and generality of sets of desirable gambles, that can be linked 
to preference relations and also encompass as particular cases coherent lower previsions or sets of probability measures.

For completeness, in this appendix we translate the results we have established in the first part of the paper to social rules defined on coherent lower previsions $\underline{P}$ or on closed convex sets of probability measures $\mathcal{M}$. We shall make use of the one-to-one correspondence between these models and coherent sets of strictly desirable gambles: in the case of a coherent lower prevision $\underline{P}$, its associated set is given by (3); given a closed convex set of probability measures $\mathcal{M}$, we consider the set of strictly desirable gambles associated with its lower prevision $\underline{P}:=\min \mathcal{M}$.

As a consequence, we shall say for instance that a social rule $\Gamma$ on coherent lower previsions satisfies weak Pareto if and only if the social rule $\Gamma^{\prime}$ that we can determine on the associated sets of strictly desirable gambles by

$$
\Gamma^{\prime}\left(\left[\mathcal{D}_{i}\right]\right)=\left\{f: \Gamma\left(\left[\underline{P}_{i}\right]\right)(f)>0\right\} \cup \mathcal{L}^{+}, \text {where } \underline{P}_{i} \text { is induced by } \mathcal{D}_{i} \text { through }(1)
$$

satisfies weak Pareto. Similar considerations hold for the other axioms, and for social rules defined on credal sets.

Our next result summarises how the different axioms and government systems are represented in this context:

Theorem 6 Let $\Gamma$ be a social rule defined on a profile $\left[\underline{P}_{i}\right]$ (resp., $\left.\left[\mathcal{M}_{i}\right]\right)$ given by coherent lower previsions (resp., credal sets). Then:

1. $\Gamma$ satisfies weak Pareto $\Leftrightarrow\left(\forall\left[\underline{P}_{i}\right] \in \mathcal{A}\right) \underline{P} \geq \min _{i} \underline{P}_{i}, \Leftrightarrow\left(\forall\left[\mathcal{M}_{i}\right] \in \mathcal{A}\right) \mathcal{M} \subseteq$ $\overline{\operatorname{ch}\left(\cup_{i} \mathcal{M}_{i}\right)}$.

2. $\quad \Gamma$ satisfies strict completeness $\Leftrightarrow\left(\forall\left[\underline{P}_{i}\right] \in \mathcal{A}\right) \underline{P}$ linear $\Leftrightarrow\left(\forall\left[\mathcal{M}_{i}\right] \in \mathcal{A}\right)|\mathcal{M}|=1$.

3. A set $\mathcal{G} \subseteq \mathcal{H}$ is decisive $\Leftrightarrow\left(\forall\left[\underline{P}_{i}\right] \in \mathcal{A}\right) \underline{P} \geq \min _{i \in \mathcal{G}} \underline{P}_{i} \Leftrightarrow\left(\forall\left[\mathcal{M}_{i}\right] \in \mathcal{A}\right) \mathcal{M} \subseteq$ $\overline{\operatorname{ch}\left(\cup_{i \in \mathcal{G}} \mathcal{M}_{i}\right)}$.

4. A set $\mathcal{G} \subseteq \mathcal{H}$ is an oligarchy $\Leftrightarrow\left(\forall\left[\underline{P}_{i}\right] \in \mathcal{A}\right) \underline{P} \geq \min _{i \in \mathcal{G}} \underline{P}_{i}$ and $\bar{P} \geq \max _{i \in \mathcal{G}} P_{i}$.

5. An individual $j \in \mathcal{H}$ is a dictator $\Leftrightarrow\left(\forall\left[\underline{P}_{i}\right] \in \mathcal{A}\right) \underline{P} \geq \underline{P}_{j} \Leftrightarrow \mathcal{M} \subseteq \mathcal{M}_{j}$.

6. $\Gamma$ satisfies anonymity $\Leftrightarrow\left(\forall\left[\underline{P}_{i}\right],\left[\underline{P}_{\sigma(i)}\right] \in \mathcal{A}\right) \underline{P}=\underline{P}_{\sigma} \Leftrightarrow\left(\forall\left[\mathcal{M}_{i}\right],\left[\mathcal{M}_{\sigma(i)}\right] \in\right.$ $\mathcal{A}) \mathcal{M}=\mathcal{M}_{\sigma}$, where $\sigma$ is a permutation of $\mathcal{H}$ and $P_{\sigma}$ (resp. $\left.\left[\mathcal{M}_{\sigma}\right]\right)$ is the coherent lower prevision (resp. credal set) given by $\Gamma\left(\left[P_{\sigma(i)}\right]\right)\left(\right.$ resp. $\left.\Gamma\left(\left[\mathcal{M}_{\sigma(i)}\right]\right)\right)$.

7. $\Gamma$ satisfies independence of irrelevant alternatives $\Leftrightarrow\left(\forall\left[\underline{P}_{i}\right],\left[\underline{P}_{i}^{\prime}\right] \in \mathcal{A}\right)\left(\forall f \notin \mathcal{L}^{+}\right)$:

$$
(\forall i \in \mathcal{H}) \underline{P}_{i}(f) \underline{P}_{i}^{\prime}(f)>0 \Rightarrow \underline{P}(f) \underline{P}^{\prime}(f)>0,
$$

where $\underline{P}^{\prime}$ denotes the coherent lower prevision that is obtained by means of $\Gamma\left(\left[\underline{P}_{i}^{\prime}\right]\right)$.

More generally speaking, if we consider a social rule $\Gamma^{\prime}$ defined on coherent sets of desirable gambles, we wonder if similar equivalences hold with respect to the social rule $\Gamma$ on coherent lower previsions or credal sets that is associated with it. However, this will not be the case in general. To see just one example, consider the social rule $\Gamma^{\prime}$ given by $\Gamma^{\prime}\left(\left[\mathcal{D}_{i}\right]\right)=\mathcal{D}_{j \triangleright}$, for some fixed $j \in \mathcal{H}$. Then $\Gamma^{\prime}$ will not satisfy weak Pareto in general (for instance with $\mathcal{D}_{i}=\mathcal{D}_{j}$ for every $i$, and the latter being non strictly desirable), but its associated $\Gamma$ will be given by $\Gamma\left(\left[\underline{P}_{i}\right]\right)=\underline{P}_{j} \geq \min _{i} \underline{P}_{i}$.

As a corollary, which follows immediately from Theorem 6 and (2), we can characterise the above conditions in the particular case where the profile is precise: 
Corollary 3 Let $\Gamma$ be a strict complete social rule defined on a profile $\left[P_{i}\right]$ given by linear previsions. Then:

1. A set $\mathcal{G} \subseteq \mathcal{H}$ is decisive $\Leftrightarrow\left(\forall\left[P_{i}\right] \in \mathcal{A}\right) \Gamma\left(\left[P_{i}\right]\right) \in \operatorname{ch}\left(\left\{P_{i} \mid i \in \mathcal{G}\right\}\right)$.

2. A set $\mathcal{G} \subseteq \mathcal{H}$ is an oligarchy $\Leftrightarrow\left(\forall\left[P_{i}\right] \in \mathcal{A}\right), P_{j}=P_{j^{\prime}} \forall j, j^{\prime} \in \mathcal{G}$ and $\Gamma\left(\left[P_{i}\right]\right)=P_{j}$.

3. An individual $j \in \mathcal{H}$ is a dictator $\Leftrightarrow\left(\forall\left[P_{i}\right] \in \mathcal{A}\right) \Gamma\left(\left[P_{i}\right]\right)=P_{j}$.

We see then that the notion of oligarchy does not make much sense in the precise case, because it forces all the individuals that form the oligarchy to have exactly the same opinion.

\section{Appendix C: Proofs}

In the following proofs, it will be convenient at times to reformulate the condition of avoiding partial loss in the following manner:

Lemma 5 Given a set $\mathcal{K}$ of desirable gambles, $\operatorname{ch}(\mathcal{K}) \cap\left(\mathcal{L}^{-} \cup\{0\}\right)=\emptyset \Leftrightarrow 0 \notin \operatorname{posi}(\mathcal{K} \cup$ $\left.\mathcal{L}^{+}\right)$. As a consequence, if any of the two conditions above hold, it follows that $\mathcal{K}$ has a coherent (maximal) superset.

Proof It is equivalent to prove that

$$
\operatorname{ch}(\mathcal{K}) \cap\left(\mathcal{L}^{-} \cup\{0\}\right) \neq \emptyset \Leftrightarrow 0 \in \operatorname{posi}\left(\mathcal{K} \cup \mathcal{L}^{+}\right) .
$$

To prove that this is the case, note that

$$
\begin{aligned}
& 0 \in \operatorname{posi}\left(\mathcal{K} \cup \mathcal{L}^{+}\right) \\
& \Leftrightarrow\left(\exists f \in \mathcal{L}^{+} \cup\{0\}, f_{j} \in \mathcal{K}, \lambda_{j}>0, j=1, \ldots, r, r \geq 1\right) 0=f+\sum_{j=1}^{r} \lambda_{j} f_{j} \\
& \Leftrightarrow\left(\exists f \in \mathcal{L}^{+} \cup\{0\}, f_{j} \in \mathcal{K}, \lambda_{j}>0, j=1, \ldots, r, r \geq 1\right)-f=\sum_{j=1}^{r} \lambda_{j} f_{j} \\
& \Leftrightarrow\left(\exists f \in \mathcal{L}^{+} \cup\{0\}, f_{j} \in \mathcal{K}, \lambda_{j}>0, j=1, \ldots, r, r \geq 1\right)-\frac{f}{\sum_{j=1}^{r} \lambda_{j}}=\sum_{j=1}^{r} \frac{\lambda_{j}}{\sum_{j=1}^{r} \lambda_{j}} f_{j},
\end{aligned}
$$

and since the left-hand side belongs to $\mathcal{L}^{-} \cup\{0\}$ and the right-hand side belongs to $\operatorname{ch}(\mathcal{K})$, we deduce that this is equivalent to $\operatorname{ch}(\mathcal{K}) \cap\left(\mathcal{L}^{-} \cup\{0\}\right) \neq \emptyset$.

For the second statement, if any of these conditions hold we deduce that the set posi $(\mathcal{K} \cup$ $\mathcal{L}^{+}$) is a coherent set of desirable gambles that trivially includes $\mathcal{K}$. Since any coherent set of desirable gambles has a coherent maximal superset [12, Corollary 4], it follows in particular that $\mathcal{K}$ has a coherent maximal superset.

In order to prove Lemmas 1 and 2, we also need first to establish the following auxiliary result:

Lemma 6 Assume that $|\mathcal{Z}| \geq 3$. Then for every gamble $f$ such that $f \notin\left(\mathcal{L}^{+} \cup \mathcal{L}^{-} \cup\{0\}\right)$ there always exist two gambles $\{h, t\}$ such that $-f=f+h+t$ and

○ $\operatorname{ch}(\{f, h\}) \cap\left(\mathcal{L}^{-} \cup\{0\}\right)=\emptyset, \operatorname{ch}(\{-f, h, f+h\}) \cap\left(\mathcal{L}^{-} \cup\{0\}\right)=\emptyset$ and $\operatorname{ch}(\{-f, h,-(f+$ h) $\}) \cap\left(\mathcal{L}^{-} \cup\{0\}\right)=\emptyset$; 
$\circ \quad \operatorname{ch}(\{f+h, t\}) \cap\left(\mathcal{L}^{-} \cup\{0\}\right)=\emptyset, \operatorname{ch}(\{-(f+h), t,-f\}) \cap\left(\mathcal{L}^{-} \cup\{0\}\right)=\varnothing$ and $\operatorname{ch}(\{-(f+h), t, f\}) \cap\left(\mathcal{L}^{-} \cup\{0\}\right)=\emptyset$.

Proof Since $f$ is such that $f \notin\left(\mathcal{L}^{+} \cup \mathcal{L}^{-} \cup\{0\}\right)$ then there exist at least two points $z_{1}, z_{2} \in \mathcal{Z}$ with $z_{1} \neq z_{2}$ such that $f\left(z_{1}\right)=f_{1}>0$ and $f\left(z_{2}\right)=f_{2}<0$. We consider a number of possibilities:

- If there is some $z_{3} \neq z_{1}, z_{2}$ such that $f\left(z_{3}\right) \geq 0$, then we define, for a fixed $\varepsilon>0$ :

$$
h(z):=\left\{\begin{array}{ll}
f_{1}>0 & \text { if } z=z_{1} \\
-f_{2} / 2>0 & \text { if } z=z_{2} \\
-2 f(z)-\varepsilon & \text { otherwise }
\end{array} \quad \text { and } \quad t(z):= \begin{cases}-3 f_{1}<0 & \text { if } z=z_{1} \\
-3 f_{2} / 2>0 & \text { if } z=z_{2} \\
\varepsilon & \text { otherwise }\end{cases}\right.
$$

Then by construction $-f=f+h+t$. To see that they fulfil the conditions in the lemma, note that:

○ $\operatorname{ch}(\{f, h\}) \cap\left(\mathcal{L}^{-} \cup\{0\}\right)=\emptyset\left(\right.$ use $\left.z_{1}\right)$

○ $\operatorname{ch}(\{-f, h, f+h\}) \cap\left(\mathcal{L}^{-} \cup\{0\}\right)=\emptyset$ : if $0 \geq \lambda_{-f}(-f)+\lambda_{h} h+\lambda_{f+h}(f+h)$, then it should be on the one hand $\lambda_{-f} \geq \lambda_{h}+2 \lambda_{f+h}$ because of $z_{1}$, and on the other $2 \lambda_{-f}+\lambda_{h} \leq \lambda_{f+h}$ because of $z_{2}$; these two conditions are incompatible.

○ $\operatorname{ch}(\{-f, h,-(f+h)\}) \cap\left(\mathcal{L}^{-} \cup\{0\}\right)=\emptyset\left(\right.$ use $\left.z_{2}\right)$;

and also

○ $\operatorname{ch}(\{f+h, t\}) \cap\left(\mathcal{L}^{-} \cup\{0\}\right)=\emptyset$ : if $\lambda_{f+h}(f+h)+\lambda_{t} t \leq 0$, it should be on the one hand $\lambda_{f+h} \leq 0.6$ because of $z_{1}$ and on the other $\lambda_{f+h} \geq 0.75$ because of $z_{2}$;

$\circ \quad \operatorname{ch}(\{-(f+h), t,-f\}) \cap\left(\mathcal{L}^{-} \cup\{0\}\right)=\emptyset\left(\right.$ use $\left.z_{2}\right)$;

$\circ \operatorname{ch}(\{-(f+h), t, f\}) \cap\left(\mathcal{L}^{-} \cup\{0\}\right)=\emptyset$ (use $z_{3}$ and $\left.z_{1}\right)$.

○ If $f\left(z_{3}\right)<0$ for every $z_{3} \neq z_{1}, z_{2}$, then we define:

$$
h(z):=\left\{\begin{array}{ll}
-f_{1}<0 & \text { if } z=z_{1} \\
-f_{2} / 2>0 & \text { if } z=z_{2} \\
-2 f(z) & \text { otherwise }
\end{array} \quad \text { and } \quad t(z):= \begin{cases}-f_{1}<0 & \text { if } z=z_{1} \\
-3 f_{2} / 2>0 & \text { if } z=z_{2} \\
0 & \text { otherwise. }\end{cases}\right.
$$

Then by construction $-f=f+h+t$. To see that they fulfil the conditions in the lemma, note that:

○ $\operatorname{ch}(\{f, h\}) \cap\left(\mathcal{L}^{-} \cup\{0\}\right)=\emptyset$ : if $\lambda_{f}(f)+\lambda_{h} h \leq 0$, it should be on the one hand $\lambda_{f} \leq 0.5$ because of $z_{1}$ and on the other $\lambda_{f} \geq \frac{2}{3}$ because of $z_{3}$;

$\circ \quad \operatorname{ch}(\{-f, h, f+h\}) \cap\left(\mathcal{L}^{-} \cup\{0\}\right)=\emptyset\left(\right.$ use $\left.z_{3}\right)$;

○ $\operatorname{ch}(\{-f, h,-(f+h)\}) \cap\left(\mathcal{L}^{-} \cup\{0\}\right)=\emptyset$ (use $\left.z_{2}\right)$;

and also

$\circ \operatorname{ch}(\{f+h, t\}) \cap\left(\mathcal{L}^{-} \cup\{0\}\right)=\emptyset\left(\right.$ use $z_{2}$ and $\left.z_{3}\right) ;$ 
$\circ \quad \operatorname{ch}(\{-(f+h), t,-f\}) \cap\left(\mathcal{L}^{-} \cup\{0\}\right)=\emptyset\left(\right.$ use $\left.z_{2}\right)$

○ $\operatorname{ch}(\{-(f+h), t, f\}) \cap\left(\mathcal{L}^{-} \cup\{0\}\right)=\emptyset$ : if $0 \geq-\lambda_{-(f+h)}(f+h)+\lambda_{t} t+\lambda_{f} f$, then it should be $\lambda_{t} \geq \lambda_{f}$ so that this combination is non-positive on $z_{1}$, but then this implies that it is positive on $z_{2}$.

Proof of Lemma 1 Note that we can assume without loss of generality that $\mathcal{G}$ is a proper subset of $\mathcal{H}$, since otherwise the thesis follows immediately from the property of weak Pareto. In addition, we can then assume that the gamble $f$ does not belong to $\mathcal{L}^{+} \cup \mathcal{L}^{-} \cup\{0\}$.

We must prove that $\left(\forall\left[\mathcal{D}_{i}\right]\right) \cap_{i \in \mathcal{G}} \mathcal{D}_{i} \subseteq \Gamma\left(\left[\mathcal{D}_{i}\right]\right)$. Consider thus a profile $\left[\mathcal{D}_{i}\right]$ (eventually in $\hat{\mathbb{D}}^{n}$ ), and let $g \in \cap_{i \in \mathcal{G}} \mathcal{D}_{i}$. We can assume without loss of generality that $g \notin \mathcal{L}^{+}$, since in that case it trivially belongs to $\Gamma\left(\left[\mathcal{D}_{i}\right]\right)$. There are a number of possibilities:

1. Assume first of all that $g=f+h$, where:

$$
\begin{gathered}
\operatorname{ch}(\{f, h\}) \cap\left(\mathcal{L}^{-} \cup\{0\}\right)=\emptyset, \\
\operatorname{ch}(\{-f, h, g\}) \cap\left(\mathcal{L}^{-} \cup\{0\}\right)=\emptyset, \\
\operatorname{ch}(\{-f, h,-g\}) \cap\left(\mathcal{L}^{-} \cup\{0\}\right)=\emptyset .
\end{gathered}
$$

Hence, it holds that:

- By Lemma 5, if $\operatorname{ch}(\{f, h\}) \cap\left(\mathcal{L}^{-} \cup\{0\}\right)=\emptyset$, there exists a coherent set $\mathcal{D}^{1}$ (possibly maximal) of desirable gambles that includes both $f, h$, and as a consequence also $g$ by additivity.

- Since $\operatorname{ch}(\{-f, h,-g\}) \cap\left(\mathcal{L}^{-} \cup\{0\}\right)=\varnothing$, then the coherent set $\mathcal{D}^{2}:=$ $\operatorname{posi}\left(\{-f, h,-g\} \cup \mathcal{L}^{+}\right.$) (or a maximal set that contains it) of desirable gambles includes $h$ and not $f$ and $g$.

○ Finally, let $\mathcal{D}^{3}:=\operatorname{posi}\left(\{-f, h, g\} \cup \mathcal{L}^{+}\right.$) (or a maximal set that contains it). It includes $h, g$ but not $f$.

Let us now define the following profile $\left[\mathcal{D}_{i}^{\prime}\right]$ :

○ For every $i \in \mathcal{G}$, let $\mathcal{D}_{i}^{\prime}=\mathcal{D}^{1}$ (possibly the maximal one). Then $f, h \in \mathcal{D}_{i}^{\prime}$, whence also $g \in \mathcal{D}_{i}^{\prime}$ by additivity.

○ Given $i \in \mathcal{H} \backslash \mathcal{G}$, if $g \notin \mathcal{D}_{i}$ then we let $\mathcal{D}_{i}^{\prime}=\mathcal{D}^{2}$ (possibly the maximal one). Then $h \in \mathcal{D}_{i}^{\prime}$ and $f, g \notin \mathcal{D}_{i}^{\prime}$.

○ Given $i \in \mathcal{H} \backslash \mathcal{G}$, if $g \in \mathcal{D}_{i}$ then we let $\mathcal{D}_{i}^{\prime}=\mathcal{D}^{3}$ (possibly the maximal one). Then $h, g \in \mathcal{D}_{i}^{\prime}$ and $f \notin \mathcal{D}_{i}^{\prime}$.

It follows then that $h \in \cap_{i} \mathcal{D}_{i}^{\prime}$, whence $h \in \Gamma\left(\left[\mathcal{D}_{i}^{\prime}\right]\right)$, using the weak Pareto property; moreover, $f \in \cap_{i \in \mathcal{G}} \mathcal{D}_{i}^{\prime}$ and $f \notin \cup_{i \in \mathcal{H} \backslash \mathcal{G}} \mathcal{D}_{i}^{\prime}$. Since $\mathcal{G}$ is almost decisive for $f$, we deduce that $f \in \Gamma\left(\left[\mathcal{D}_{i}^{\prime}\right]\right)$. Since the latter is a coherent set of desirable gambles, we deduce by additivity that $g=f+h \in \Gamma\left(\left[\mathcal{D}_{i}^{\prime}\right]\right)$. And since $\pi_{g}\left(\left[\mathcal{D}_{i}\right]\right)=\pi_{g}\left(\left[\mathcal{D}_{i}^{\prime}\right]\right)$ by construction, we deduce from the independence of irrelevant alternatives that also $g \in \Gamma\left(\left[\mathcal{D}_{i}\right]\right)$.

Now note that the reasoning above can be applied in particular for those $g \gtrless f$ (i.e., when $h \in \mathcal{L}^{+}$). To see that this is the case, note that when $h \in \mathcal{L}^{+}$:

(C.1) $\operatorname{ch}(\{f, h\}) \cap\left(\mathcal{L}^{-} \cup\{0\}\right)=\emptyset$ is equivalent to $\{f\}$ avoiding partial loss, which holds because $f \notin \mathcal{L}^{+} \cup \mathcal{L}^{-} \cup\{0\}$ by assumption.

(C.2) $\operatorname{ch}(\{-f, h, g\}) \cap\left(\mathcal{L}^{-} \cup\{0\}\right)=\emptyset$ becomes equivalent to $\operatorname{ch}(\{-f, g\}) \cap\left(\mathcal{L}^{-} \cup\right.$ $\{0\})=\varnothing$. But if this intersection was non-empty we would deduce that for 
some $\lambda_{-f}+\lambda_{g}=1$ it holds that $0 \geq \lambda_{-f}(-f)+\lambda_{g} g \geq\left(\lambda_{g}-\lambda_{-f}\right) f$, which contradicts the assumption that $f \notin \mathcal{L}^{+} \cup \mathcal{L}^{-} \cup\{0\}$.

(C.3) $\operatorname{ch}(\{-f, h,-g\}) \cap\left(\mathcal{L}^{-} \cup\{0\}\right)=\emptyset$ becomes equivalent to $\{-g\}$ avoiding partial loss, which holds because it cannot be $g \in \mathcal{L}^{-} \cup\{0\}$ since we are assuming that $g \in \cap_{i \in \mathcal{G}} \mathcal{D}_{i}$.

This allows us to deduce that

$$
\mathcal{G} \text { is decisive for any } g \gtrless f \text {. }
$$

Next we establish that:

$$
\mathcal{G} \text { almost decisive for } g \Rightarrow \mathcal{G} \text { decisive for }-g \text {. }
$$

To prove this, note that we can assume without loss of generality that $g \notin \mathcal{L}^{+} \cup$ $\mathcal{L}^{-} \cup\{0\}$. Applying Lemma 6, we can find two gambles $h, t$ such that $-g=g+h+t$. Moreover, by construction in the proof of that lemma we have that $\operatorname{ch}(\{g, h\}) \cap\left(\mathcal{L}^{-} \cup\right.$ $\{0\})=\emptyset, \operatorname{ch}(\{-g, h,(g+h)\}) \cap\left(\mathcal{L}^{-} \cup\{0\}\right)=\emptyset$ and $\operatorname{ch}(\{-g, h,-(g+h)\}) \cap\left(\mathcal{L}^{-} \cup\right.$ $\{0\})=\emptyset$. Applying point 1 , we deduce that $\mathcal{G}$ is decisive for $g+h$, and therefore also almost decisive. Moreover, we also have from the proof of the lemma that $\operatorname{ch}(\{g+$ $h, t\}) \cap\left(\mathcal{L}^{-} \cup\{0\}\right)=\emptyset, \operatorname{ch}(\{-(g+h), t,-g\}) \cap\left(\mathcal{L}^{-} \cup\{0\}\right)=\emptyset$ and $\operatorname{ch}(\{-(g+$ $h), t, g\}) \cap\left(\mathcal{L}^{-} \cup\{0\}\right)=\emptyset$. Applying again point 1 , we deduce that $\mathcal{G}$ is decisive for $g+h+t=-g$.

Thus, (C.5) holds. Applying twice this condition we deduce in particular that $\mathcal{G}$ is decisive for $-f$, and also for $f$.

2. Assume now that $g=f+h$, with $h \in \mathcal{L}^{-}$. Then $g \lesseqgtr f$, or, equivalently $-f \lesseqgtr-g$. Since $\mathcal{G}$ is decisive at $-f$, it is in particular almost decisive at this gamble, whence by (C.4) $\mathcal{G}$ is also almost decisive at $-g \gtrless-f$. Applying now (C.5), we deduce that $\mathcal{G}$ is also decisive at $g$.

3. Finally, consider a gamble $h \notin \mathcal{L}^{+} \cup \mathcal{L}^{-} \cup\{0\}$, and let $g:=f+h$. Then we can rewrite $g$ as $g=f-h^{-}+h^{+}$, where $h^{+}$and $h^{-}$are respectively the positive and the negative part function of $h$, i.e. $h^{+}:=\max \{0, h\}$ and $h^{-}:=-\min \{0, h\}$. It then follows from point 2 that $\mathcal{G}$ is decisive on $g=f-h^{-}$because $-h^{-} \in \mathcal{L}^{-}$, and as a consequence it is also almost decisive on this gamble. If we now apply (C.4) we deduce that $\mathcal{G}$ is decisive on $g=\left(f-h^{-}\right)+h^{+} \gtrless f-h^{-}$.

Proof of Lemma 2 Let us prove that there is a proper subset $\mathcal{G}^{\prime}$ of $\mathcal{G}$ that is also decisive. For this, we consider a partition of $\mathcal{G}$ into non-empty and disjoint subsets $\mathcal{G}_{1}$ and $\mathcal{G}_{2}$, and we proceed to establish that one of these two sets is also decisive.

Consider $z_{1}, z_{2} \in \mathcal{Z}$ with $z_{1} \neq z_{2}$, and let us define the gambles $f_{1}, f_{2}, f_{3}$ by:

$$
f_{1}(z):=\left\{\begin{array}{ll}
-1 & \text { if } z=z_{1} \\
1 & \text { if } z=z_{2} \\
0 & \text { otherwise, }
\end{array} \quad f_{2}(z):=\left\{\begin{array}{ll}
0 & \text { if } z=z_{1} \\
-2 & \text { if } z=z_{2} \\
1 & \text { otherwise, }
\end{array} \quad f_{3}:=f_{1}+f_{2} .\right.\right.
$$

Let $z_{3}$ denote an element different from $z_{1}, z_{2}$, existing because $|\mathcal{Z}| \geq 3$. These gambles satisfy the following conditions:

○ $\operatorname{ch}\left(\left\{f_{1}, f_{2}, f_{3}\right\}\right) \cap\left(\mathcal{L}^{-} \cup\{0\}\right)=\emptyset$ (use $z_{3}$ and $\left.z_{2}\right)$;

○ $\operatorname{ch}\left(\left\{-f_{1}, f_{2},-f_{3}\right\}\right) \cap\left(\mathcal{L}^{-} \cup\{0\}\right)=\emptyset$ (use $z_{1}$ and $\left.z_{3}\right)$;

○ $\operatorname{ch}\left(\left\{f_{1},-f_{3}\right\}\right) \cap\left(\mathcal{L}^{-} \cup\{0\}\right)=\emptyset$ (use $\left.z_{2}\right)$. 
Then, thanks to Lemma 5 we can consider the following profile $\left[\hat{\mathcal{D}}_{i}\right] \in \hat{\mathbb{D}}^{n}$ :

○ for all $i \in \mathcal{G}_{1}, \hat{\mathcal{D}}_{i}:=M_{1} \supseteq \operatorname{posi}\left(\left\{f_{1}, f_{2}, f_{3}\right\} \cup \mathcal{L}^{+}\right)$, with $M_{1} \in \hat{\mathbb{D}}$;

○ for all $i \in \mathcal{G}_{2}, \hat{\mathcal{D}}_{i}:=M_{2} \supseteq \operatorname{posi}\left(\left\{-f_{1}, f_{2},-f_{3}\right\} \cup \mathcal{L}^{+}\right)$, with $M_{2} \in \hat{\mathbb{D}}$;

○ for all $i \notin \mathcal{G}, \hat{\mathcal{D}}_{i}:=M_{3} \supseteq \operatorname{posi}\left(\left\{f_{1},-f_{3}\right\} \cup \mathcal{L}^{+}\right)$, with $M_{3} \in \hat{\mathbb{D}}$.

Note then that $-f_{1} \notin \hat{\mathcal{D}}_{i}$ for any $i \notin \mathcal{G}_{2}$, and that $f_{3} \notin \hat{\mathcal{D}}_{i}$ for any $i \notin \mathcal{G}_{1}$, because these sets of gambles are maximal. Since $f_{2} \in \cap_{i \in \mathcal{G}} \hat{\mathcal{D}}_{i}$, it follows from decisiveness of $\mathcal{G}$ that $f_{2} \in \Gamma\left(\left[\hat{\mathcal{D}}_{i}\right]\right)$. Since this set is maximal, then it either includes $f_{1}$ or $-f_{1}$.

1. If $f_{1} \in \Gamma\left(\left[\hat{\mathcal{D}}_{i}\right]\right)$, then $f_{3} \in \Gamma\left(\left[\hat{\mathcal{D}}_{i}\right]\right)$ by additivity. Let us prove that in that case $\mathcal{G}_{1}$ is almost decisive with respect to $f_{3}$.

Consider any profile $\left[\hat{\mathcal{D}}_{i}^{\prime}\right] \in \hat{\mathbb{D}}^{n}$, such that $f_{3} \in \cap_{i \in \mathcal{G}_{1}} \hat{\mathcal{D}}_{i}^{\prime}$ and $f_{3} \notin \cup_{i \notin \mathcal{G}_{1}} \hat{\mathcal{D}}_{i}^{\prime}$. Then $\pi_{f_{3}}\left(\left[\hat{\mathcal{D}}_{i}^{\prime}\right]\right)=\pi_{f_{3}}\left(\left[\hat{\mathcal{D}}_{i}\right]\right)$, and applying the independence of irrelevant alternatives we deduce that $\mathcal{G}_{1}$ is almost decisive on $f_{3}$. Applying Lemma 1 , we deduce that it is also decisive.

2. If $-f_{1} \in \Gamma\left(\left[\mathcal{D}_{i}\right]\right)$, then let us show that $\mathcal{G}_{2}$ is almost decisive for $-f_{1}$.

Consider any profile $\left[\hat{\mathcal{D}}_{i}^{\prime}\right] \in \hat{\mathbb{D}}^{n}$, such that $-f_{1} \in \cap_{i \in \mathcal{G}_{2}} \hat{\mathcal{D}}_{i}^{\prime}$ and $-f_{1} \notin \cup_{i \notin \mathcal{G}_{2}} \hat{\mathcal{D}}_{i}^{\prime}$. It follows that $\pi_{-f_{1}}\left(\left[\hat{\mathcal{D}}_{i}^{\prime}\right]\right)=\pi_{-f_{1}}\left(\left[\hat{\mathcal{D}}_{i}\right]\right)$, and applying the independence of irrelevant alternatives we deduce that $-f_{1} \in \Gamma\left(\left[\hat{\mathcal{D}}_{i}^{\prime}\right]\right)$. Thus, $\mathcal{G}_{2}$ is almost decisive on $-f_{1}$, and applying Lemma 1 we deduce that it is also decisive.

Proof of Theorem 1 The case of $n=1$ is trivial, so let us assume $n \geq 2$. By weak Pareto condition we know that $\mathcal{H}$ is decisive. By repeatedly applying Lemma 2 , we can eventually arrive to a decisive individual, who must, thus, be a dictator.

Proof of Proposition 1 Let $j$ be the dictator. Since we have unlimited maximal domain, the social rule must be given by $\Gamma\left(\left[\mathcal{D}_{i}\right]\right)=\mathcal{D}_{j}$ for any profile $\left[\mathcal{D}_{i}\right]$, and as a consequence it satisfies independence of irrelevant alternatives.

Proof of Lemma 3 Suppose $\mathcal{G}$ and $\mathcal{G}^{\prime}$ are both oligarchies, and assume that $i^{*} \in \mathcal{G}^{\prime} \backslash \mathcal{G}$. Let $f \notin \mathcal{L}^{+} \cup \mathcal{L}^{-} \cup\{0\}$, and let us consider a profile (if needed made up only by maximal coherent sets of desirable gambles) $\left[\mathcal{D}_{i}\right]$ such that $f \in \mathcal{D}_{i}$ for all $i \in \mathcal{G}$ and $-f \in \mathcal{D}_{i}$ for all $i \in \mathcal{G}^{\prime} \backslash \mathcal{G}$ : it suffices to make

$$
\mathcal{D}_{i}:= \begin{cases}\operatorname{posi}\left(\{f\} \cup \mathcal{L}^{+}\right) & \text {or a maximal superset, if } i \in \mathcal{G} \\ \operatorname{posi}\left(\{-f\} \cup \mathcal{L}^{+}\right) & \text {or a maximal superset, if } i \notin \mathcal{G} .\end{cases}
$$

Then since $\mathcal{G}^{\prime}$ is an oligarchy we should have $f \notin \Gamma\left(\left[\mathcal{D}_{i}\right]\right)$, but $\mathcal{G}$ decisive implies $f \in$ $\Gamma\left(\left[\mathcal{D}_{i}\right]\right)$, a contradiction.

Proof of Theorem 2 Since by Lemma 3 there can be at most one oligarchy, we only need to establish its existence.

By the weak Pareto condition, the set $\mathcal{H}$ of all individuals is decisive. Let $\mathcal{G}$ be a decisive set of minimal size, meaning that there does not exist $\mathcal{G}^{\prime} \subset \mathcal{G}$ that is also decisive. We shall demonstrate that $\mathcal{G}$ is an oligarchy. If $\mathcal{G}$ contains a single member, then $\mathcal{G}$ is trivially an oligarchy because any dictatorship is. Let us then consider the case in which $|\mathcal{G}| \geq 2$. Note 
that we only need to prove that $\mathrm{O} 2$ holds, because $\mathrm{O} 1$ follows immediately because $\mathcal{G}$ is decisive.

We consider first of all the case where $\Gamma$ has unlimited domain.

Consider a profile $\left[\mathcal{D}_{i}\right]$, and let $f \in \cup_{i \in \mathcal{G}} \mathcal{D}_{i}$. We must prove that $-f \notin \Gamma\left(\left[\mathcal{D}_{i}\right]\right)$. We may assume without loss of generality that $f \notin \mathcal{L}^{+} \cup \mathcal{L}^{-} \cup\{0\}$; the result otherwise is trivial. We can partition the group $\mathcal{G}$ into the following sets:

○ $A:=\left\{i \in \mathcal{G} \mid f \in \mathcal{D}_{i}\right\}$,

○ $B:=\left\{i \in \mathcal{G} \mid-f \in \mathcal{D}_{i}\right\}$,

○ $C:=\left\{i \in \mathcal{G} \mid f \notin \mathcal{D}_{i},-f \notin \mathcal{D}_{i}\right\}$,

where by assumption $A$ is non-empty, but $B, C$ may be.

Since $f \notin\left(\mathcal{L}^{+} \cup \mathcal{L}^{-} \cup\{0\}\right)$, there exist two points $z_{1}, z_{2} \in \mathcal{Z}$ with $z_{1} \neq z_{2}$ such that $f\left(z_{1}\right)=f_{1}>0$ and $f\left(z_{2}\right)=f_{2}<0$. Let us prove that there always exist two gambles $\{h, g\}$ such that $f=h+g$ and

○ $\operatorname{ch}(\{f, h\}) \cap\left(\mathcal{L}^{-} \cup\{0\}\right)=\emptyset$, and $g,-g \notin \mathcal{D}^{1}:=\operatorname{posi}\left(\{f, h\} \cup \mathcal{L}^{+}\right)$;

○ $\operatorname{ch}(\{-f, h\}) \cap\left(\mathcal{L}^{-} \cup\{0\}\right)=\emptyset$, and $-g \in \mathcal{D}^{2}:=\operatorname{posi}\left(\{-f, h\} \cup \mathcal{L}^{+}\right)$;

○ $\operatorname{ch}(\{-g, h\}) \cap\left(\mathcal{L}^{-} \cup\{0\}\right)=\emptyset$, and $f,-f \notin \mathcal{D}^{3}:=\operatorname{posi}\left(\{-g, h\} \cup \mathcal{L}^{+}\right)$;

○ $\operatorname{ch}(\{-f\}) \cap\left(\mathcal{L}^{-} \cup\{0\}\right)=\emptyset$, whence $-g$ and as a consequence $h \notin \mathcal{D}^{4}:=\operatorname{posi}(\{-f\} \cup$ $\left.\mathcal{L}^{+}\right)$.

We consider a number of possibilities:

1. If there is some $z_{3} \neq z_{1}, z_{2}$ such that $f\left(z_{3}\right)>0$, then we define:

$$
h(z):=\left\{\begin{array}{ll}
3 f_{1} / 2>0 & \text { if } z=z_{1} \\
f_{2} / 2<0 & \text { if } z=z_{2} \\
f(z)-\varepsilon & \text { otherwise }
\end{array} \quad \text { and } \quad g(z):= \begin{cases}-f_{1} / 2<0 & \text { if } z=z_{1} \\
f_{2} / 2<0 & \text { if } z=z_{2} \\
\varepsilon & \text { otherwise }\end{cases}\right.
$$

where $\varepsilon>0$ is small enough for the conditions to be satisfied.

Then by construction $f=h+g$. To see that they fulfil the conditions, note that:

○ $\operatorname{ch}(\{f, h\}) \cap\left(\mathcal{L}^{-} \cup\{0\}\right)=\emptyset\left(\right.$ use $\left.z_{1}\right) ;$

- $g \notin \mathcal{D}^{1}$ (use $z_{1}$ );

- $\quad-g \notin \mathcal{D}^{1}$ (use $z_{3}$ with $0<\varepsilon<f\left(z_{3}\right)$ );

$\circ \operatorname{ch}(\{-f, h\}) \cap\left(\mathcal{L}^{-} \cup\{0\}\right)=\emptyset$ : If $\lambda_{-f}(-f)+\lambda_{h} h \leq 0$ it should be on the one hand $\lambda_{-f} \geq 0.6$ because of $z_{1}$ and on the other $\lambda_{-f} \leq \frac{1}{3}$ because of $z_{2}$. Then $-g \in \mathcal{D}^{2}$ by additivity;

○ $\operatorname{ch}(\{-g, h\}) \cap\left(\mathcal{L}^{-} \cup\{0\}\right)=\emptyset\left(\right.$ use $\left.z_{1}\right)$;

- $\quad f \notin \mathcal{D}^{3}$ : If $f \geq \lambda_{-g}(-g)+\lambda_{h} h$ for some non-negative $\lambda_{-g}, \lambda_{h}$ (with at least one positive), we have that

$$
f_{2} \geq-\lambda_{-g} f_{2} / 2+\lambda_{h} f_{2} / 2
$$

whence

$$
-1 \geq \lambda_{-g} / 2-\lambda_{h} / 2
$$

dividing by the positive number $-f_{2}$. This means that

$$
\lambda_{h} \geq 2+\lambda_{-g} \geq 2 .
$$


Now, this means that

$$
\lambda_{h} h\left(z_{1}\right)+\lambda_{-g}\left(-g\left(z_{1}\right)\right)=\lambda_{h} 3 f_{1} / 2+\lambda_{-g} f_{1} / 2 \geq 3 f_{1}>f_{1},
$$

a contradiction;

- $\quad-f \notin \mathcal{D}^{3}$ (use $z_{1}$ );

○ $\operatorname{ch}(\{-f\}) \cap\left(\mathcal{L}^{-} \cup\{0\}\right)=\emptyset$ by definition of $f$;

- $\quad-g \notin \mathcal{D}^{4}$ : If $-g \geq \lambda(-f)$ it should be on the one hand $\lambda \leq 0.5$ because of $z_{2}$ and on the other $\lambda \geq \varepsilon / f\left(z_{3}\right)>0.5$ if we choose $0<f\left(z_{3}\right) / 2<\varepsilon<f\left(z_{3}\right)$, because of $z_{3}$.

2. If there is some $z_{3} \neq z_{1}, z_{2}$ such that $f\left(z_{3}\right)=0$, then we define:

$$
h(z):=\left\{\begin{array}{ll}
3 f_{1} / 2>0 & \text { if } z=z_{1} \\
f_{2} / 2<0 & \text { if } z=z_{2} \\
f(z)-\varepsilon & \text { otherwise }
\end{array} \quad \text { and } \quad g(z):= \begin{cases}-f_{1} / 2<0 & \text { if } z=z_{1} \\
f_{2} / 2<0 & \text { if } z=z_{2} \\
\varepsilon & \text { otherwise }\end{cases}\right.
$$

where $\varepsilon>0$ is small enough for the conditions to be satisfied.

Notice that $h$ and $g$ are the same of the previous case, so to show that they fulfil the conditions we indicate only the cases that involve the value of $f\left(z_{3}\right)$.

○ It only changes $-g \notin \mathcal{D}^{1}$ : If $-g \geq \lambda_{f} f+\lambda_{h} h$ for some non-negative $\lambda_{f}, \lambda_{h}$ (with at least one positive), we have that

$$
-\varepsilon \geq \lambda_{f}\left(f\left(z_{3}\right)\right)+\lambda_{h}\left(f\left(z_{3}\right)-\varepsilon\right)=-\varepsilon \lambda_{h},
$$

from which follows $\lambda_{h} \geq 1$, which does not work for $z_{1}$ because $-g\left(z_{1}\right)<h\left(z_{1}\right)$ and $h\left(z_{1}\right), f_{1}$ are both positive;

- same as before;

- same as before;

○ it only changes $-g \notin \mathcal{D}^{4}$ (use $z_{3}$ ).

3 Finally, if $f\left(z_{3}\right)<0$ for every $z_{3} \neq z_{1}, z_{2}$, then we define:

$$
h(z):=\left\{\begin{array}{ll}
f_{1}>0 & \text { if } z=z_{1} \\
3 f_{2} / 2<0 & \text { if } z=z_{2} \\
0 & \text { otherwise }
\end{array} \text { and } g(z):= \begin{cases}0 & \text { if } z=z_{1} \\
-f_{2} / 2>0 & \text { if } z=z_{2} \\
f(z) & \text { otherwise. }\end{cases}\right.
$$

Then by construction $f=h+g$. To see that they fulfil the conditions, note that:

$\circ \quad \operatorname{ch}(\{f, h\}) \cap\left(\mathcal{L}^{-} \cup\{0\}\right)=\emptyset\left(\right.$ use $\left.z_{1}\right) ;$

- $g \notin \mathcal{D}^{1}$ (use $z_{1}$ );

- $\quad-g \notin \mathcal{D}^{1}$ (use $z_{1}$ );

$\circ \quad \operatorname{ch}(\{-f, h\}) \cap\left(\mathcal{L}^{-} \cup\{0\}\right)=\varnothing$ (use $z_{3}$ and $\left.z_{1}\right)$ and $-g \in \mathcal{D}_{2}$ by additivity;

○ $\operatorname{ch}(\{-g, h\}) \cap\left(\mathcal{L}^{-} \cup\{0\}\right)=\varnothing\left(\right.$ use $z_{3}$ and $\left.z_{1}\right)$;

- $f \notin \mathcal{D}^{3}$ (use $z_{3}$ );

- $\quad-f \notin \mathcal{D}^{3}$ (use $z_{1}$ );

○ $\operatorname{ch}(\{-f\}) \cap\left(\mathcal{L}^{-} \cup\{0\}\right)=\emptyset$ by definition of $f$;

- $\quad-g \notin \mathcal{D}^{4}$ : (use $z_{2}$ ). 
Let us consider now a profile $\left[\mathcal{D}_{i}^{\prime}\right.$ ] where $\mathcal{D}_{i}^{\prime}=\mathcal{D}^{1}$ if $i \in A, \mathcal{D}_{i}^{\prime}=\mathcal{D}^{2}$ if $i \in B$, $\mathcal{D}_{i}^{\prime}=\mathcal{D}^{3}$ if $i \in C$ and $\mathcal{D}_{i}^{\prime}$ is either $\mathcal{L}^{+}$or $\mathcal{D}^{4}$ if $i \notin \mathcal{G}$ (as needed below so as to include $-f$ if necessary). We shall use these sets to establish that $-f \notin \Gamma\left(\left[\mathcal{D}_{i}\right]\right)$. We have a number of possible scenarios:

- If $A, B, C$ are all non-empty and $-f \in \Gamma\left(\left[\mathcal{D}_{i}\right]\right)$, then we can consider a profile $\left[\mathcal{D}_{i}^{\prime}\right]$ as above so that $\pi_{-f}\left(\left[\mathcal{D}_{i}\right]\right)=\pi_{-f}\left(\left[\mathcal{D}_{i}^{\prime}\right]\right)$. Applying independence of irrelevant alternatives, it follows that $-f \in \Gamma\left(\left[\mathcal{D}_{i}^{\prime}\right]\right)$. Since $\mathcal{G}$ is decisive and $h \in \cap_{i \in \mathcal{G}} \mathcal{D}_{i}^{\prime}$, we also have that $h \in \Gamma\left(\left[\mathcal{D}_{i}^{\prime}\right]\right)$, we deduce that $-g=-f+h$ belongs to $\Gamma\left(\left[\mathcal{D}_{i}^{\prime}\right]\right)$. By construction, $\pi_{-g}\left(\left[\mathcal{D}_{i}^{\prime}\right]\right)=\left(\overrightarrow{1}_{B \cup C}\right)$. Applying independence of irrelevant alternatives, for any other profile $\left[\mathcal{D}_{i}^{\prime \prime}\right]$ such that $-g \in \cap_{i \in B \cup C} \mathcal{D}_{i}^{\prime \prime}$ and $-g \notin \cup_{i \notin B \cup C} \mathcal{D}_{i}^{\prime \prime}$, it holds that $-g \in \Gamma\left(\left[\mathcal{D}_{i}^{\prime \prime}\right]\right)$. But this means that $B \cup C$ is almost decisive with respect to $g$, and applying Lemma 1 , it is decisive. This contradicts that $\mathcal{G}$ is a decisive set of minimal size.

- If $A \neq \emptyset \neq B$ and $C=\emptyset$, we reason as in the previous case, and end up concluding that $B$ is a decisive set.

- If $A \neq \emptyset \neq C$ and $B=\emptyset$, we reason as in the first case, and end up concluding that $C$ is a decisive set.

○ Finally, if $A \neq \emptyset$ and $B=C=\emptyset$, it holds that $f \in \cap_{i \in \mathcal{G}} \mathcal{D}_{i}$, whence, since $\mathcal{G}$ is decisive, $f \in \Gamma\left(\left[\mathcal{D}_{i}\right]\right)$. Since the latter is a coherent set, this means that $-f \notin \Gamma\left(\left[\mathcal{D}_{i}\right]\right)$.

We consider next the case of unlimited maximal domain. Recall that since $\mathcal{G}$ is decisive, condition $\mathrm{O} 1$ is satisfied. Let us consider next condition $\mathrm{O} 2$. Consider a profile $\left[\hat{\mathcal{D}}_{i}\right] \in \hat{\mathbb{D}}^{n}$. Assume that $f \in \cup_{i \in \mathcal{G}} \hat{\mathcal{D}}_{i}$. Since all the sets in the profile are maximal, we can partition again the group $\mathcal{G}$ into the following sets:

○ $A:=\left\{i \in \mathcal{G} \mid f \in \hat{\mathcal{D}}_{i}\right\}$,

○ $B:=\left\{i \in \mathcal{G} \mid-f \in \hat{\mathcal{D}}_{i}\right\}$,

where by assumption $A$ is non-empty, but $B$ may be. We need to show that $-f \notin \Gamma\left(\left[\hat{\mathcal{D}}_{i}\right]\right)$. There are two possibilities:

○ if both $A, B$ are non-empty and $-f \in \Gamma\left(\left[\hat{\mathcal{D}}_{i}\right]\right)$, applying independence of irrelevant alternatives, for any other profile $\left[\hat{\mathcal{D}}_{i}^{\prime}\right]$ such that $-f \in \cap_{i \in B} \hat{\mathcal{D}}_{i}^{\prime}$ and $-f \notin \cup_{i \in A} \hat{\mathcal{D}}_{i}^{\prime}$, it holds that $-f \in \Gamma\left(\left[\hat{\mathcal{D}}_{i}^{\prime}\right]\right)$. But this means that $B$ is almost decisive with respect to $-f$, and applying Lemma 1 , it is decisive. This contradicts that $\mathcal{G}$ is a decisive set of minimal size;

○ if instead $A \neq \emptyset$ and $B=\emptyset$, it holds that $f \in \cap_{i \in \mathcal{G}} \hat{\mathcal{D}}_{i}$, whence, since $\mathcal{G}$ is decisive, $f \in \Gamma\left(\left[\hat{\mathcal{D}}_{i}\right]\right)$. Since the latter is a coherent set, this means that $-f \notin \Gamma\left(\left[\hat{\mathcal{D}}_{i}\right]\right)$.

Proof of Proposition 2 Assume ex-absurdo that there exists a profile $\left[\mathcal{D}_{i}\right]$ such that $\Gamma\left(\left[\mathcal{D}_{i}\right]\right) \supsetneq \cap_{i \in \mathcal{G}} \mathcal{D}_{i}$, and let us consider $f \in \Gamma\left(\left[\mathcal{D}_{i}\right]\right) \backslash \cap_{i \in \mathcal{G}} \mathcal{D}_{i}$, so that $\emptyset \neq A=\{i \in \mathcal{G}$ : $\left.f \in \mathcal{D}_{i}\right\} \neq \mathcal{G}$. For every $j \in \mathcal{G} \backslash A$, let $\mathcal{D}_{j}^{\prime}$ be a maximal set of desirable gambles that includes $-f$, and let $\mathcal{D}_{i}^{\prime}:=\mathcal{D}_{i}$ for every $i \in A \cup \mathcal{G}^{c}$. Then since $-f \in \cup_{i \in \mathcal{G}} \mathcal{D}_{i}^{\prime}$, it follows from $\mathrm{O} 2$ that $f \notin \Gamma\left(\left[\mathcal{D}_{i}^{\prime}\right]\right)$. But on the other hand $f$ belongs to the same sets in the profiles $\left[\mathcal{D}_{i}\right]$ and $\left[\mathcal{D}_{i}^{\prime}\right]$, so $f \in \Gamma\left(\left[\mathcal{D}_{i}\right]\right), f \notin \Gamma\left(\left[\mathcal{D}_{i}^{\prime}\right]\right)$ is a contradiction with independence of irrelevant alternatives. 
Proof of Theorem 3 From Theorem 2 there exists a unique oligarchy $\mathcal{G}$. Then we must show that if the social rule satisfies also anonymity, $\mathcal{G}=\mathcal{H}$. Assume ex-absurdo that $\mathcal{G} \neq \mathcal{H}$, and take $i^{*} \notin \mathcal{G}$.

By the hypothesis of unlimited (maximal) domain we can consider a profile (possibly composed only by maximal coherent sets of desirable gambles) $\left[\mathcal{D}_{i}\right]$ such that $f \in \mathcal{D}_{i^{*}}$ and $-f \in \mathcal{D}_{j} \forall j \neq i^{*}$. Since $\mathcal{G}$ is an oligarchy, it follows that $-f \in \Gamma\left(\left[\mathcal{D}_{i}\right]\right)$.

Consider now a permutation $\sigma$ of $\mathcal{H}$ such that $\sigma\left(i^{*}\right) \neq i^{*}, \sigma\left(i^{*}\right) \in \mathcal{G}$, and let $\left[\mathcal{D}_{i}^{\prime}\right]$ : $=\left[\mathcal{D}_{\sigma(i)}\right]$ denote the associated profile. Then there exists some $j \in \mathcal{G}$ such that $f \in$ $\mathcal{D}_{j}^{\prime}$, whence, by definition of oligarchy, $-f \notin \Gamma\left(\left[\mathcal{D}_{i}^{\prime}\right]\right)$. But this means that $\Gamma\left(\left[\mathcal{D}_{\sigma(i)}\right]\right) \neq$ $\Gamma\left(\left[\mathcal{D}_{i}\right]\right)$, meaning that anonymity is violated.

Proof of Proposition 3 Thanks to Proposition 2 we know that for every profile (possibly composed only by maximal coherent sets of desirable gambles) $\left[\mathcal{D}_{i}\right], \Gamma\left(\left[\mathcal{D}_{i}\right]\right)=\cap_{i} \mathcal{D}_{i}$. Hence it satisfies anonymity.

Proof of Lemma 4 That $\mathcal{E}$ is coherent follows from [35, Prop. 29].

From (6), any $f \in \mathcal{E}$ can be written as

$$
f=\sum_{i \in \mathcal{H}} \lambda_{i} \mathbb{I}_{i} \otimes f_{i}+f_{0},
$$

with $f_{0} \in \mathcal{L}^{+}(\mathcal{H} \times \mathcal{Z}) \cup\{0\}, f_{i} \in \mathcal{D}_{i} \cup\{0\}, \lambda_{i}>0, f \neq 0$. Then $f \in \operatorname{Marg}_{\mathcal{Z}}(\mathcal{E})$ iff $f \in \mathcal{E}$ and

$(\forall i, j \in \mathcal{H})(\forall z \in \mathcal{Z}) f(i, z):=\lambda_{i} \mathbb{I}_{i} \otimes f_{i}(i, z)+f_{0}(i, z)=\lambda_{j} \mathbb{I}_{j} \otimes f_{j}(j, z)+f_{0}(j, z)=: f(j, z)$.

This is equivalent to

$$
(\forall i, j \in \mathcal{H})(\forall z \in \mathcal{Z}) \lambda_{i} f_{i}(z)+f_{0}(i, z)=\lambda_{j} f_{j}(z)+f_{0}(j, z) .
$$

You can observe that, fixing $i$ and $j$, the left term of this equation is a gamble in $\mathcal{D}_{i}$ and the right term is a gamble in $\mathcal{D}_{j}$, considering also that $f \neq 0$. Thus, $f \in \operatorname{Marg}_{\mathcal{Z}}(\mathcal{E})$ if and only if it depends only on $z \in \mathcal{Z}$ and, as a function of only $z \in \mathcal{Z}$, it belongs to $\cap_{i \in \mathcal{H}} \mathcal{D}_{i}$. Hence we have the thesis.

Proof of Theorem 4 Let us address the points of the statement in turn.

1. The equality of the sets in (6) and Theorem 4 is well known, see for instance [35, Prop. 29].

2. The converse implication is trivial: if such an $\mathcal{E}^{\prime}$ exists, then $\Gamma\left(\left[\mathcal{D}_{i}\right]\right)=\operatorname{Marg}_{\mathcal{Z}}\left(\mathcal{E}^{\prime}\right) \supseteq$ $\operatorname{Marg}_{\mathcal{Z}}(\mathcal{E})$.

For the direct implication, consider $\mathcal{E}^{\prime}$ as defined in (7). It includes $\mathcal{E}$ by definition. To prove that it is coherent, note that $\mathcal{E}^{\prime}=\operatorname{posi}\left(\Gamma\left(\left[\mathcal{D}_{i}\right]\right) \otimes \mathcal{H} \cup \cup_{i} \mathcal{D} \mid i\right)$ (this is due again to the transformation referenced in point 1). It includes $\mathcal{L}^{+}(\mathcal{H} \times \mathcal{Z})$ because for every $f \in \mathcal{L}^{+}(\mathcal{H} \times \mathcal{Z})$, the restriction of $\mathbb{I}_{i} \otimes f$ on $\mathcal{L}(\mathcal{Z})$ belongs to $\mathcal{D}_{i} \cup\{0\}$ for all $i \in \mathcal{H}$. Thus, coherence holds if and only if $0 \notin \operatorname{posi}\left(\Gamma\left(\left[\mathcal{D}_{i}\right]\right) \otimes \mathcal{H} \cup \cup_{i} \mathcal{D} \mid i\right)$.

To prove that this is the case, let us reason by contradiction. Note that the zero gamble can in principle be produced only by adding $f_{0} \otimes \mathcal{H}$ with $\sum_{i \in \mathcal{H}} \mathbb{I}_{i} \otimes f_{i}$, for some $f_{0} \in \Gamma\left(\left[\mathcal{D}_{i}\right]\right) \cup\{0\},(\forall i \in \mathcal{H}) f_{i} \in \mathcal{D}_{i} \cup\{0\}$, not all of them zero. In order for the sum to yield zero, $f_{0}$ must be different from zero. And since $f_{0} \otimes \mathcal{H}$ is $\mathcal{Z}$ measurable, in order to yield zero it must hold that $f_{1}=\cdots=f_{n}=-f_{0}$. This implies that $-f_{0} \in \cap_{i \in \mathcal{H}} \mathcal{D}_{i}$. We are showing the direct implication, so it follows by hypothesis 
$\Gamma\left(\left[\mathcal{D}_{i}\right]\right) \supseteq \operatorname{Marg}_{\mathcal{Z}}(\mathcal{E})$ and Lemma 4, that $-f_{0} \in \Gamma\left(\left[\mathcal{D}_{i}\right]\right)$. But then $\Gamma\left(\left[\mathcal{D}_{i}\right]\right)$ contains both $f_{0}$ and $-f_{0}$. This contradicts the coherence of $\Gamma\left(\left[\mathcal{D}_{i}\right]\right)$. Therefore the sum of two gambles in $\mathcal{E}^{\prime}$ must be different from zero.

To conclude, we show that $\Gamma\left(\left[\mathcal{D}_{i}\right]\right)=\operatorname{Marg}_{\mathcal{Z}}\left(\mathcal{E}^{\prime}\right)$. Since the direct inclusion is trivial, we focus on the converse inclusion. Remember that $\operatorname{Marg}_{\mathcal{Z}}\left(\mathcal{E}^{\prime}\right)=\mathcal{E}^{\prime} \cap \mathcal{L}_{\mathcal{Z}}(\mathcal{H} \times$ $\mathcal{Z}$ ). Let us consider a $\mathcal{Z}$-measurable gamble $f \in \mathcal{E}^{\prime}: f=f_{0} \otimes \mathcal{H}+\sum_{i \in \mathcal{H}} \mathbb{I}_{i} \otimes f_{i}$. The case $f_{1}=\cdots=f_{n}=0$ is trivial, so let us assume that there is $i \in \mathcal{H}$ such that $f_{i} \neq 0$. Since $f$ is $\mathcal{Z}$-measurable, this means that

$$
(\forall i, j \in \mathcal{H})(\forall z \in \mathcal{Z}) f(i, z)=f_{0}(z)+f_{i}(z)=f_{0}(z)+f_{j}(z)=f(j, z) .
$$

This implies $f_{j}=f_{i}$ for all $j \neq i$. As a consequence, $f_{i} \in \operatorname{Marg}_{\mathcal{Z}}(\mathcal{E})$ and by hypothesis then $f_{i} \in \Gamma\left(\left[\mathcal{D}_{i}\right]\right)$. It follows that $f_{0}+f_{1}+\cdots+f_{n} \in \Gamma\left(\left[\mathcal{D}_{i}\right]\right)$, whence $f \in \Gamma\left(\left[\mathcal{D}_{i}\right]\right)$.

3. Assume by contradiction that there is such a set $\mathcal{E}^{\prime \prime}: \mathcal{E}^{\prime} \supsetneq \mathcal{E}^{\prime \prime} \supseteq \mathcal{E}$. Since $\mathcal{E}^{\prime}$ strictly contains $\mathcal{E}^{\prime \prime}$, there must be some $f_{0} \in \Gamma\left(\left[\mathcal{D}_{i}\right]\right)$ such that $f_{0} \otimes \mathcal{H} \notin \mathcal{E}^{\prime \prime}$. Note that $f_{0} \notin \cap_{i \in \mathcal{H}} \mathcal{D}_{i}$, otherwise it would belong to $\mathcal{E}^{\prime \prime}$ given that $\mathcal{E}^{\prime \prime} \supseteq \mathcal{E}$. Whence $f_{0} \in$ $\Gamma\left(\left[\mathcal{D}_{i}\right]\right) \backslash \cap_{i \in \mathcal{H}} \mathcal{D}_{i}$ and by marginalising $\mathcal{E}^{\prime \prime}$ we obtain a set that does not contain $f_{0}$; therefore $\Gamma\left(\left[\mathcal{D}_{i}\right]\right) \neq \operatorname{Marg}_{\mathcal{Z}}\left(\mathcal{E}^{\prime \prime}\right)$. This is a contradiction.

4. Remember that $\mathcal{E}^{\prime} \mid i=\left\{f \in \mathcal{E}^{\prime}: f=\mathbb{I}_{i} f\right\}$. Since $f_{0} \otimes \mathcal{H}$, in the definition of $\mathcal{E}^{\prime}$, is constant on the elements of $\mathcal{H}$, any gamble $f=\mathbb{I}_{i} f$ must be such that $f_{0}=0$. The thesis then follows immediately.

5. Remember again that $\operatorname{Marg}_{\mathcal{H}}\left(\mathcal{E}^{\prime}\right)=\mathcal{E}^{\prime} \cap \mathcal{L}_{\mathcal{H}}(\mathcal{H} \times \mathcal{Z})$. Let us consider a $\mathcal{H}$-measurable gamble $f \in \mathcal{E}^{\prime}: f=f_{0} \otimes \mathcal{H}+\sum_{i \in \mathcal{H}} \mathbb{I}_{i} \otimes f_{i}$ for some $f_{0} \in \Gamma\left(\left[\mathcal{D}_{i}\right]\right) \cup\{0\},(\forall i \in$ $\mathcal{H}) f_{i} \in \mathcal{D}_{i} \cup\{0\}$, not all of them zero. $\mathcal{H}$-measurability requires that

$$
(\forall i \in \mathcal{H})\left(\forall z, z^{\prime} \in \mathcal{Z}\right) f(i, z)=f_{0}(z)+f_{i}(z)=f_{0}\left(z^{\prime}\right)+f_{i}\left(z^{\prime}\right)=f\left(i, z^{\prime}\right) .
$$

This is valid if and only if $f_{i}+f_{0}=k_{i}$ constant $\forall i \in \mathcal{H}$. Hence we have that $f \in$ $\operatorname{Marg}_{\mathcal{H}}\left(\mathcal{E}^{\prime}\right)$ is a gamble $f \in \mathcal{E}^{\prime}: f=f_{0} \otimes \mathcal{H}+\sum_{i \in \mathcal{H}} \mathbb{I}_{i} \otimes f_{i}$ such that $f_{i}(z)=$ $-f_{0}(z)+k_{i}$ for all $z \in \mathcal{Z}$, where $k_{i}$ is a constant for all $i \in \mathcal{H}$.

Now we can distinguish two cases:

(a) If $f_{0}(z)=k$ for all $z \in \mathcal{Z}$, then it must be $k \geq 0$, considering that $f_{0} \in \Gamma\left(\left[\mathcal{D}_{i}\right]\right) \cup$ $\{0\}$. It then follows that $f_{i}(z)=k_{i}-k$ for all $z \in \mathcal{Z}$, for all $i \in \mathcal{H}$, and it must be $k_{i}-k \geq 0$, since otherwise $f_{i} \in \mathcal{L}^{-}(\mathcal{Z})$ and this would contradict $f_{i} \in \mathcal{D}_{i} \cup\{0\}$. We deduce that $(\forall i \in \mathcal{H})(\forall z \in \mathcal{Z}) f(i, z)=k+k_{i}-k \geq 0$ and so $f \in \mathcal{L}^{+}(\mathcal{H})$. Note that $f \neq 0$ otherwise $f \notin \mathcal{E}^{\prime}$.

(b) Assume next that $f_{0}$ is not constant (and in particular non-zero). This means that it must be $f_{i}=k_{i}-f_{0} \neq 0$ for every $i \in \mathcal{H}$, and in particular $f_{i} \in \mathcal{D}_{i}$ for every $i$.

For all $i \in \mathcal{H}$ we can define

$$
\bar{P}_{i}\left(f_{0}\right):=\inf \left\{\lambda \in \mathbb{R}: \lambda-f_{0} \in \mathcal{D}_{i}\right\},
$$

the upper prevision of $f_{0}$ obtained from $\mathcal{D}_{i}$. Thus, $k_{\underline{i}} \geq \bar{P}_{i}\left(f_{0}\right)$, for all $i \in \mathcal{H}$, and the equality can only hold if for some $i \in \mathcal{H}, \bar{P}_{i}\left(f_{0}\right)$ is a mininum, i.e. if $\bar{P}_{i}\left(f_{0}\right)-f_{0} \in \mathcal{D}_{i}$. This means that, given that $f(i, z)=k_{i}$ for every $i \in \mathcal{H}, z \in \mathcal{Z}$,

$$
f(i, z) \geq \bar{P}_{i}\left(f_{0}\right),
$$

and that the inequality is strict unless $\bar{P}_{i}\left(f_{0}\right)-f_{0} \in \mathcal{D}_{i}$. Now, given the fourth statement, we know that for every $i \in \mathcal{H}, g \in \mathcal{L}(\mathcal{Z})$,

$$
\bar{P}_{i}(g)=\bar{P}(g \mid i) \text {, }
$$


so, defining the conditional upper prevision $\bar{P}(g \mid \mathcal{H}):=\sum_{i} \mathbb{I}_{i} \bar{P}(g \mid i)$, we have that $f \geq \bar{P}\left(f_{0} \mid \mathcal{H}\right)$, and that the inequality is strict unless $\bar{P}\left(f_{0} \mid i\right)-f_{0} \in \mathcal{D}_{i}$.

This establishes the form of $\operatorname{Marg}_{\mathcal{H}}\left(\mathcal{E}^{\prime}\right)$.

Proof of Corollary 1 Weak Pareto means that if a gamble $f$ is strictly desirable for any $P_{i}$, $i=1, \ldots, n$, then it should also be strictly desirable for $P$, or, equivalently, that $P \geq$ $\min _{i} P_{i}$ (Theorem 6(1)). But this means that $P$ belongs to the credal set associated with the coherent lower prevision $\underline{P}:=\min _{i} P_{i}$, and as a consequence that it is a convex combination of $P_{1}, \ldots, P_{n}$.

Proof of Theorem 5 If $\pi$ is not degenerate, then we can find $j_{1} \neq j_{2}$ in $\mathcal{H}$ such that $\pi\left(j_{1}\right), \pi\left(j_{2}\right)>0$. Consider a profile $\left[\hat{\mathcal{D}}_{i}\right] \in \mathcal{A}$ such that $\hat{\mathcal{D}}_{i}$ assigns all the mass to $\left(s_{1}, x_{1}\right)$ if $i=j_{1}$ and all the mass to $\left(s_{2}, x_{2}\right)$ if $i \neq j_{1}$, where $s_{1} \neq s_{2}$ and $j_{1} \neq j_{2}$. Then we obtain

$$
P\left(s_{1}, x_{1}\right)=\pi\left(j_{1}\right)=P\left(s_{1}\right)=P\left(x_{1}\right),
$$

meaning that the linear prevision induced by $\Gamma\left(\left[\hat{\mathcal{D}}_{i}\right]\right)$ is not state independent. This is a contradiction.

Proof of Corollary 2 1. It follows from Theorem 2 and definition of weak dictatorship.

2. This is a consequence of Theorem 1 and definition of strong dictatorship.

3. It follows from Theorem 3 and definition of weak dictatorship.

Proof of Theorem 6 We shall establish the first equivalence; the second equivalence follows taking into account that

$$
\underline{P} \geq \underline{Q} \Leftrightarrow \mathcal{M}(\underline{P}) \subseteq \mathcal{M}(\underline{Q}),
$$

that the credal set associated with $\min _{i} \underline{P}_{i}$ is $\overline{\operatorname{ch}\left(\cup_{i} \mathcal{M}\left(\underline{P}_{i}\right)\right)}$ and that the one associated with $\max _{i} \underline{P}_{i}$ is $\cap_{i} \mathcal{M}\left(\underline{P}_{i}\right)$, where the credal set associated with a coherent lower prevision is given by (2).

We shall denote by $\mathcal{D}_{i}$ the coherent set of strictly desirable gambles associated with $\underline{P}_{i}$ by means of (3), and by $\mathcal{D}$ the coherent set of strictly desirable gambles associated with $\underline{P}=\Gamma\left(\left[P_{i}\right]\right)$.

1. Let us establish the direct implication. Assume that $\Gamma$ satisfies weak Pareto, and as a consequence that $\cap_{i \in \mathcal{H}} \mathcal{D}_{i} \subseteq \Gamma^{\prime}\left(\left[\mathcal{D}_{i}\right]\right)$, where $\Gamma^{\prime}$ is associated with $\Gamma$ by means of (9). It follows that, for any $f \in \mathcal{L}$,

$$
\underline{P}(f):=\sup \left\{\mu \in \mathbb{R}: f-\mu \in \Gamma^{\prime}\left[\mathcal{D}_{i}\right]\right\} \geq \sup \left\{\mu \in \mathbb{R}: f-\mu \in \cap_{i \in \mathcal{H}} \mathcal{D}_{i}\right\} .
$$

Let us prove that the right-hand side is greater than or equal to $\min _{i} \underline{P}_{i}(f)$. Assume that $\min _{i} \underline{P}_{i}(f)=\underline{P}_{j}(f)$. Then it follows from (1) that for any $\varepsilon>0, f-\underline{P}_{j}(f)+\varepsilon$ belongs to $\mathcal{D}_{j}$, and for any $j^{\prime} \neq j$ it holds that $f-\underline{P}_{j}(f)+\varepsilon \geq f-\underline{P}_{j^{\prime}}(f)+\varepsilon \in \mathcal{D}_{j^{\prime}}$. As a consequence, $f-\underline{P}_{j}(f)+\varepsilon \in \cap_{i} \mathcal{D}_{i}$, whence

$$
\sup \left\{\mu \in \mathbb{R}: f-\mu \in \cap_{i \in \mathcal{H}} \mathcal{D}_{i}\right\} \geq \underline{P}_{j}(f)-\varepsilon .
$$

Since this holds for any $\varepsilon>0$, it follows that

$$
\sup \left\{\mu \in \mathbb{R}: f-\mu \in \cap_{i \in \mathcal{H}} \mathcal{D}_{i}\right\} \geq \underline{P}_{j}(f)=\min _{i} \underline{P}_{i}(f) .
$$

We conclude that $\underline{P}(f) \geq \min _{i} \underline{P}_{i}(f)$ for every gamble $f$. 
To see the converse, given a gamble $f \in\left(\cap_{i} \mathcal{D}_{i}\right) \backslash \mathcal{L}^{+}$, it holds that $\underline{P}_{i}(f)>0$ for every $i$, whence $0<\min \underline{P}_{i}(f) \leq \underline{P}(f)$, and as a consequence $f$ belongs to the set of strictly desirable gambles associated with $\underline{P}$. Since trivially $\left(\cap_{i} \mathcal{D}_{i}\right) \cap \mathcal{L}^{+} \subseteq \mathcal{L}^{+} \subseteq \mathcal{D}$, we deduce that $\Gamma^{\prime}$ satisfies weak Pareto and therefore so does $\Gamma$.

2. This follows from (1) and (3) and the definition of strict completeness and maximal strict desirability.

3. This follows applying the first statement to $\mathcal{G}$ instead of $\mathcal{H}$.

4. The first part is a consequence of the third statement. Let us now prove that

$$
\left.\left[(\exists i \in \mathcal{G})\left(f \in \mathcal{D}_{i}\right)\right) \Rightarrow-f \notin \Gamma^{\prime}\left(\left[\mathcal{D}_{i}\right]\right)\right] \Leftrightarrow \bar{P} \geq \max _{i \in \mathcal{G}} \underline{P}_{i} .
$$

To see the direct implication, consider a gamble $f$, and let $\underline{P}_{j}(f)=\max _{i \in \mathcal{G}} \underline{P}_{i}(f)$. If $\underline{P}_{j}(f)>0$, then $f \in \mathcal{D}_{j}$, whence $-f \notin \Gamma^{\prime}\left(\left[\mathcal{D}_{i}\right]\right)$ and, from (1) and the conjugacy relation $\bar{P}(\cdot)=-\underline{P}(-\cdot)$, it follows that $\bar{P}(f) \geq 0$. Moreover, it must be $\bar{P}(f)>0$ : otherwise by considering $f^{\prime}:=f-\frac{\underline{P}_{j}(f)}{2}$, we would obtain $\underline{P}_{j}\left(f^{\prime}\right)>0$ and $\bar{P}\left(f^{\prime}\right)<$ 0 . Therefore, we conclude that $\max _{i \in \mathcal{G}} \underline{P}_{i}(f)>0 \Rightarrow \bar{P}(f)>0$. But since both $\max _{i \in \mathcal{G}} \underline{P}_{i}$ and $\bar{P}$ satisfy constant additivity, this means that $\bar{P} \geq \max _{i \in \mathcal{G}} \underline{P}_{i}$.

Conversely, if there is a gamble $f$ such that $f \in \mathcal{D}_{j}$ for some $j \in \mathcal{G}$ while $-f \in \Gamma^{\prime}\left(\left[\mathcal{D}_{i}\right]\right)$, it necessarily must be $f \notin \mathcal{L}^{+}$. It then follows that $\max _{i \in \mathcal{G}} \underline{P}_{i}(f) \geq$ $\underline{P}_{j}(f)>0$, while $0<\underline{P}(-f)=-\bar{P}(f)$, meaning that $\bar{P}(f)<0$. This is a contradiction.

As a consequence, (C.6) holds. This concludes the proof of this statement.

5. This is a particular case of the third statement with $|\mathcal{G}|=1$.

6. $\Gamma$ satisfies anonymity if and only if $\Gamma^{\prime}\left(\left[\mathcal{D}_{i}\right]\right)=\Gamma^{\prime}\left(\left[\mathcal{D}_{\sigma(i)}\right]\right)$. It follows that, for any $f \in \mathcal{L}$,

$$
\underline{P}(f):=\sup \left\{\mu \in \mathbb{R}: f-\mu \in \Gamma^{\prime}\left(\left[\mathcal{D}_{i}\right]\right)\right\}=\sup \left\{\mu \in \mathbb{R}: f-\mu \in \Gamma^{\prime}\left(\left[\mathcal{D}_{\sigma(i)}\right]\right)\right\}=: \underline{P}_{\sigma}(f) .
$$

To see the converse, let us suppose without loss of generality that there is a gamble $f \in$ $\Gamma^{\prime}\left(\left[\mathcal{D}_{i}\right]\right) \cap\left(\Gamma^{\prime}\left(\left[\mathcal{D}_{\sigma(i)}\right]\right)^{c}\right.$ for some permutation $\sigma$ of $\mathcal{H}$. Then it follows that $\underline{P}(f)>0$ and $\underline{P}_{\sigma}(f) \leq 0$ and this contradicts $\underline{P}(f)=\underline{P}_{\sigma}(f)$.

7. Let us consider $f \notin \mathcal{L}^{+}$and two profiles $\left[\underline{P}_{i}\right],\left[\underline{P}_{i}^{\prime}\right]$ such that $(\forall i \in \mathcal{H}) \underline{P}_{i}(f) \underline{P}_{i}^{\prime}(f)>$ 0 . If $\Gamma$ satisfies independence of irrelevant alternatives, then $f \in \Gamma^{\prime}\left(\left[\mathcal{D}_{i}\right]\right) \Leftrightarrow \Gamma^{\prime}\left(\left[\mathcal{D}_{i}^{\prime}\right]\right)$. As a consequence, we have $\underline{P}(f)>0 \Leftrightarrow \underline{P}^{\prime}(f)>0$, whence $\underline{P}(f) \underline{P}^{\prime}(f)>0$.

Conversely, if $\underline{P}(f) \underline{P}^{\prime}(f)>0$ then $f \in \Gamma^{\prime}\left(\left[\mathcal{D}_{i}\right]\right) \Leftrightarrow \Gamma^{\prime}\left(\left[\mathcal{D}_{i}^{\prime}\right]\right)$ by (3), whence $\Gamma$ satisfies independence of irrelevant alternatives.

Acknowledgements We acknowledge the financial support of projects PGC2018-098623-B-I00. We would also like to thank Seamus Bradley, Teddy Seidenfeld, Franz Dietrich and the anonymous reviewers for stimulating discussion as well as for providing some relevant references.

Funding Open Access funding provided by Università della Svizzera italiana.

Open Access This article is licensed under a Creative Commons Attribution 4.0 International License, which permits use, sharing, adaptation, distribution and reproduction in any medium or format, as long as you give appropriate credit to the original author(s) and the source, provide a link to the Creative Commons licence, and indicate if changes were made. The images or other third party material in this article are included in the article's Creative Commons licence, unless indicated otherwise in a credit line to the material. If material is not included in the article's Creative Commons licence and your intended use is not permitted by statutory regulation or exceeds the permitted use, you will need to obtain permission directly from the copyright holder. To view a copy of this licence, visit http://creativecommons.org/licenses/by/4.0/. 


\section{References}

1. Aczél, J., Wagner, C.: A characterization of weighted arithmetic means. SIAM J. Algebraic Discret. Methods 1(3), 259-260 (1980)

2. Arrow, K.: A difficulty in the concept of social welfare. J. Polit. Econ. 58(4), 328-346 (1950)

3. Arrow, K.: Social Choice and Individual Values. Wiley (1951)

4. Arrow, K.J., Sen, A., Suzumura, K.: Handbook of Social Choice and Welfare, vol. 2. Elsevier (2010)

5. Augustin, T., Coolen, F., de Cooman, G., Troffaes, M. (eds.): Introduction to Imprecise Probabilities. Wiley, New York (2014)

6. Barthelemy, J.P.: Arrow's theorem: unusual domains and extended codomains. Math. Soc. Sci. 3, 79-89 (1982)

7. Benavoli, A., Facchini, A., Zaffalon, M.: Bernstein's socks, polynomial-time provable coherence and entaglement. In: Proceedings of ISIPTA'2019, volume 103 of Proceedings of Machine Learning Research, pp. 23-31 (2019)

8. Benavoli, A., Facchini, A., Zaffalon, M.: Computational complexity and the nature of quantum mechanics. CoRR, arXiv:1902.03513 (2019)

9. Chevaleyre, Y., Endriss, U., Lang, J., Maudet, M.: A short introduction to computational social choice. In: van Leeuwen, J., Italiano, G.F., van der Hoek, W., Meinel, C., Sack, H., Plasil, F. (eds.) Proceedings of SOFSEM 2007, 33rd Conference on Current Trends in Theory and Practice of Computer Science, volume 4362 of Lecture Notes in Computer Science, pp. 51-69. Springer (2007)

10. Cooke, R.M., Bedford, T.: Expert Opinion. Cambridge Program for Industry (1996)

11. de Cooman, G.: Belief models: an order-theoretic investigation. Ann. Math. Artif. Intell. 45, 5-34 (2005)

12. de Cooman, G., Quaeghebeur, E.: Exchangeability and sets of desirable gambles. Int. J. Approx. Reason. 53, 363-395. Special issue in honour of Henry E. Kyburg Jr (2012)

13. Dietrich, F., List, C.: Judgement aggregation with consistency alone. Technical Report METEOR Research Memorandum No. 021, METEOR, Maastricht University School of Business and Economics. https://cris.maastrichtuniversity.nl/ws/portalfiles/portal/1415140/guid-3b81b5a2-eeb7-4595-8087-e2f1e 7df5e85-ASSET1.0.pdf (2007)

14. Dietrich, F., List, C.: Judgment aggregation without full rationality. Soc. Choice Welf. 31, 15-39 (2008)

15. Dietrich, F., List, C.: Probabilistic opinion pooling generalized. Part one: General agendas. Soc. Choice Welf. 48(4), 747-786 (2017)

16. Dietrich, F., List, C.: Probabilistic opinion pooling generalized. Part two: The premise-based approach. Soc. Choice Welf. 48(4), 787-814 (2017)

17. Dubois, D., Fargier, H., Perny, P.: On the limitations of ordinal approaches to decision-making. In: Fensel, D., Giunchiglia, F., McGuinness, D.L., Williams, M.-A. (eds.) Proceedings of the Eights International Conference on Principles and Knowledge Representation and Reasoning (KR-02), Toulouse, pp. 133-146 (2002)

18. Farber, D.: The problematics of the Pareto principle. SSRN Electronic Journal (2003)

19. Feldman, A.M., Serrano, R.: Welfare Economics and Social Choice Theory. Springer Science \& Business Media (2006)

20. Fishburn, P.: Impossibility theorems without the social completeness axiom. Econometrica 42, 695-704 (1974)

21. Gärdenfors, P.: A representation theorem for voting with logical consequences. Econ. Philos. 22(2), 181-190 (2006)

22. Gibbard, A.: Social choice and the Arrow conditions. Technical report, Harvard University. http://www-personal.umich.edu/ gibbard/Gibbard1968-Social-Choice-Arrow-Conditions.pdf (1968)

23. Goddin, R.E., List, C.: Special majorities rationalized. Br. J. Polit. Sci. 36(2), 213-241 (2006)

24. Goodman, J.: Existence of compromises in simple group decisions. PhD thesis (1988)

25. Jain, R.: A note on the Arrow's impossibility theorem. Econ. Ann. 60, 39-48 (2015)

26. Kelly, J.S.: Arrow impossibility theorems. Academic Press (1978)

27. Kelly, J.S.: Social Choice Theory: An Introduction. Springer (1988)

28. Kyburg, H.E., Pittarelli, M.: Some problems for convex Bayesians. In: Dubois, D., Wellman, M.P. (eds.) Uncertainty in Artificial Intelligence: Proceedings of the Eighth conference (UAI-1992), pp. 149-154. Morgan Kaufmann Publishers (1992)

29. Lehrer, K., Wagner, C.: Probability amalgamation and the independence issue: A reply to Laddaga. Synthese 55(3), 339-346 (1983)

30. Lindley, D.V., Tversky, A., Brown, R.V.: On the reconciliation of probability assessments. J. R. Stat. Soc. Ser. A 142(2), 146-162 (1979) 
31. List, C., Pettit, P.: Aggregating sets of judgments An impossibility result. Econ. Philos. 18(1), 89-110 (2002)

32. Mas-Colell, A., Sonnenschein, H.: General possibility theorems for group decisions. Rev. Econ. Stud. 39, 165-192 (1972)

33. McConway, K.J.: Marginalization and linear opinion pools. J. Am. Stat. Assoc. 76(374), 410-414 (1981)

34. Miranda, E., Zaffalon, M.: Notes on desirability and conditional lower previsions. Ann. Math. Artif. Intell. 60(3-4), 251-309 (2010)

35. Miranda, E., Zaffalon, M., de Cooman, G.: Conglomerable natural extension. Int. J. Approx. Reason. 53(8), 1200-1227 (2012)

36. Mongin, P.: Spurious unanimity and the Pareto principle. Technical Report 1(5), London School of Economics, The Centre for Philosophy of Natural and Social Science (CPNSS) (2005)

37. Moral, S., Del Sagrado, J.: Aggregation of imprecise probabilities. In: Aggregation and Fusion of Imperfect Information, pp. 162-188. Springer (1998)

38. Nash, J.F.: The bargaining problem. Econometrica 18, 155-162 (1950)

39. Nau, R.: The aggregation of imprecise probabilities. J. Stat. Plann. Infer. 105, 265-282 (2002)

40. Nehring, K., Puppe, C.: Strategy-proof social choice on single-peakeddomains Possibility, impossibility and the space between. Technical report University of California at Davies (2002)

41. Ng, K.C., Abramson, B.: Consensus diagnosis: a simulation study. IEEE Trans. Syst. Man Cybern. 26(3), 324-339 (1996)

42. Nguyen, H.T., Kosheleva, O., Kreinovich, V.: Decision making beyond arrow's impossibility theorem, with the analysis of effects of collusion and mutual attraction. Int. J. Intell. Syst. 24, 27-47 (2009)

43. Pini, M.S., Rossi, F., Venable, K.B., Walsh, T.: Aggregating partially ordered preferences. J. Log. Comput. 19(3), 475-502 (2009)

44. Raiffa, H.: Decision Analysis. Addison-Wesley (1968)

45. Rossi, F., Venable, K.B., Walsh, T.: A Short Introduction to Preferences: Between Artificial Intelligence and Social Choice. Synthesis Lectures on Artificial Intelligence and Machine Learning. Morgan \& Claypool Publishers (2011)

46. Seidenfeld, T., Kadane, J.B., Schervish, M.J.: On the shared preferences of two Bayesian decision makers. J. Philos. 86(5), 225-244 (1989)

47. Sen, A.: Social choice theory. In: Arrow, K.J., Intriligator, M.D. (eds.) Handbook of Mathematical Economics, volume III, chapter, vol. 22, pp. 1073-1181 (1986)

48. Stewart, R., Quintana, I.O.: Probabilistic opinion pooling with imprecise probabilities. J. Philos. Log. 47(1), 17-45 (2018)

49. Stone, M.: The opinion pool. Ann. Math. Stat. 32, 1339-1342 (1961)

50. Tideman, T.N.: Independence of clones as a criterion for voting rules. Soc. Choice Welf. 4(3), 185-206 (1987)

51. Van Camp, A.: Choice Functions as a Tool to Model Uncertainty. PhD thesis, University of Ghent (2018)

52. Van Camp, A., de Cooman, G., Miranda, E., Quaeghebeur, E.: Coherent choice functions, desirability and indifference. Fuzzy Set. Syst. 341(C), 1-36 (2018)

53. Walley, P.: The elicitation and aggregation of beliefs. Technical report, University of Warwick, 1982. Statistics Research Report 23

54. Walley, P.: Statistical Reasoning with Imprecise Probabilities. Chapman and Hall, London (1991)

55. Walley, P.: Towards a unified theory of imprecise probability. Int. J. Approx. Reason. 24, 125-148 (2000)

56. Weymark, J.A.: Arrow's theorem with social quasi-orderings. Publ. Choice 42(3), 235-246 (1984)

57. Williams, P.M.: Notes on conditional previsions. Technical report, School of Mathematical and Physical Science, University of Sussex, UK, 1975. Reprinted in [58]

58. Williams, P.M.: Notes on conditional previsions. Int. J. Approx. Reason. 44, 366-383. Revised journal version of [57] (2007)

59. Zaffalon, M., Miranda, E.: Probability and time. Artif. Intell. 198(1), 1-51 (2013)

60. Zaffalon, M., Miranda, E.: Axiomatising incomplete preferences through sets of desirable gambles. J. Artif. Intell. Res. 60, 1057-1126 (2017)

61. Zaffalon, M., Miranda, E.: Desirability foundations of robust rational decision making. Synthese (2018)

Publisher's note Springer Nature remains neutral with regard to jurisdictional claims in published maps and institutional affiliations. 